\title{
Clifford and composed foliations
}

\author{
Julia Carolina Torres Lozano
}

DisSERTAÇÃO APRESENTADA

$\mathrm{AO}$

Instituto De MatemÁticA E EstatísticA

DA

Universidade de SÃo PAUlo

PARA

OBTENÇÃO DO TÍTULO

$\mathrm{DE}$

Mestre em CiÊnCIAS

Programa: Matemática

Orientador: Prof. Dr. Claudio Gorodski

Durante o desenvolvimento deste trabalho o autor recebeu auxílio financeiro da CNPq.

Beneficiaria COLFUTURO 2015

São Paulo, agosto de 2017 


\section{Clifford and composed foliations}

Esta versão da dissertação contém as correções e alterações sugeridas pela Comissão Julgadora durante a defesa da versão original do trabalho, realizada em 11/08/2017. Uma cópia da versão original está disponível no Instituto de Matemática e Estatística da Universidade de São Paulo.

Comissão Julgadora:

- Prof. Dr. Claudio Gorodski (orientador) - IME-USP

- Prof. Dr. Nikolai Alexandrovitch Goussevskii - UFMG

- Prof. Dr. Ruy Tojeiro de Figueiredo Júnior - UFSCar 


\section{Agradecimentos}

Ao término deste processo, cabe-me agradecer, embora a lista nao seja exaustiva, aos que contribuíram de diferentes maneiras para a realização deste trabalho.

Ao meu orientador, Claudio Gorodski, pela oportunidade, apoio, confiança, paciência, comprometimento e exigência; pelas reuniões semanais, a visão matemática esclarecedora, e a leitura cuidadosa e crítica do texto. Meu especial reconhecimento tanto pela compreensão dos meu limites pessoais e acadêmicos, quanto pela motivação e o desafio do meu intelecto a diário. Sem dúvida o seu conhecimento e sua experiência estão espalhadas por todas a linhas desta dissertação.

Ao professor Marcos Alexandrino, que foi fundamental no entendimento a profundidade das folheações de Clifford, minha gratidão pelo tempo dedicado. Aos professores da banca, Nikolai e Ruy, pelo sincero interesse neste trabalho e as sugestões para o documento final. Aos meus estimados amigos, Benigno e Hengameh, que em particular lhes agradeço por me ajudarem no estudo das folheações Riemannianas singulares.

Aos meu pais, Esperanza e Alejandro, pelo amor, por me ensinarem a beleza do conhecimento e do valor do trabalho com esforço e paixão, e pelo amparo incondicional para vencer as adversidades. À minha irmã e melhor amiga, Marisol, por nossa amizade infinita, pela companhia indispensável, pelo seu carinho ilimitado. À memória dos meus avós.

Aos meus amigos queridos, Alirio, Gustavo, Pablo, Laura, Carlos, Adriana, Andrés, Raibel, Diego, Marcelo, Hugo, Camilo e Lorena, que em diferentes momentos, intensidades e formas, estiveram aí para mim, me acompanhando e me reconfortando. Compartilhamos o devir dos dias, as alegrias, tristezas e desabafos, celebramos sucessos e trilhamos nossos caminhos juntos. Meu mais sentido apreço, espero ter retribuido com um pouco do que vocês merecem.

Por seus cuidados e carinhos, sou muito grata à minha mãe no Brasil, Rita, e à Hérica; à Eloisa, aos seus pais e ao Márcio pela acolhida, afeto e hospitalidade. Aos meus prezados amigos dos bandejões, dos ônibus e do portão 3 da universidade, pelos sorrisos no dia a dia e por ser tão afáveis e bondadosos comigo.

À USP e ao IME por me aceitarem no mestrado em matemática; ao CNPq pelo sustento econômico; e ao Brasil, minha segunda casa, sempre levarei esse lindo país no meu coração. 


\section{Resumo}

Torres, L. J. C. Folheações de Clifford e folheações compostas. 2017. 120 f. Dissertação (Mestrado) - Instituto de Matemática e Estatística, Universidade de São Paulo, São Paulo, 2017.

Folheações Riemannianas singulares em esferas fornecem modelos locais para folheações Riemannianas singulares mais gerais, cuja teoria contribui na compreensão de variedades Riemannianas. Daí a sua importança de estudá-los e classificá-los, uma área de pesquisa que se mantém aberta. Em 2014, Marco Radeschi construiu folheações Riemannianas singulares indecomponíveis de codimensão arbitrária, a maioria delas não homogêneas, que generalizaram todos os exemplos conhecidos desse tipo até então. A presente dissertação é um estudo detalhado desse trabalho, junto com observações sobre avanços que se têm feito neste dinâmico campo desde a publicação do artigo. Após introduzir as noções e exemplos preliminares de folheações Riemannianas singulares, ações isométricas e teoria de Clifford, é explorada uma construção de hipersuperfícies isoparamétricas não homogêneas, devida a Ferus, Karcher e Münzner (FKM), que foi peça fundamental para os resultados de Radeschi. Em seguida, descreve-se minuciosamente a construção de folheações composta e de Clifford em esferas, que são os exemplos que o autor mencionado anteriormente gerou usando sistemas de Clifford. Continuando com a análise dessas novas folheações Riemannianas singulares, estabelece-se uma extraordinária correspondência biunívoca entre folheações de Clifford (objetos meramente geométricos) e sistemas de Clifford (objetos puramente algébricos). Este texto termina examinando as relações das propriedades de homogeneidade entre folheações FKM, compostas e de Clifford.

Palavras-chave: Folheação Riemanniana singular, Folheação de Clifford, Folheação composta, Folheação FKM, Sistema de Clifford, Álgebra de Clifford. 


\section{Abstract}

Torres, L. J. C. Clifford and composed foliations. 2017. 120 f. Dissertação (Mestrado) - Instituto de Matemática e Estatística, Universidade de São Paulo, São Paulo, 2017.

Singular Riemannian foliations in spheres provide local models for an extensive kind of singular Riemannian foliations, whose theory contributes in the understanding of Riemannian manifolds. Hence the importance of studying and classifying them, a research subject that still remains open. In 2014, Marco Radeschi constructed indecomposable singular Riemannian foliations of arbitrary codimension, most of them inhomogeneous, which generalized all known examples of that type so far. The present dissertation is a detailed study of his work, along with observations about the progress made on this dynamic field since that paper was published. Besides introducing preliminary notions and examples on singular Riemannian foliations, isometric actions and Clifford theory, it is explained a construction of inhomogeneous isoparametric hypersurfaces, due to Ferus, Karcher and Münzner, that was a fundamental framework for the results of Radeschi. After that, it is described exhaustively the construction of Clifford and composed foliations in spheres, which are the examples that Radeschi created using Clifford systems. In the sequel it is established an extraordinary bijective correspondence between Clifford foliations (merely geometric objects) and Clifford systems (purely algebraic objects). This text finishes examining the relations of homogeneity properties among FKM, Clifford and composed foliations.

Keywords: Singular Riemannian foliation, Clifford foliation, Composed foliation, FKM foliation, Clifford system, Clifford algebra. 


\section{Contents}

Introduction $\quad 1$

1 Algebraic and geometric preliminaries 5

1.1 Singular Riemannian foliations . . . . . . . . . . . . . . . . 5

1.2 Clifford systems, their algebras and representations . . . . . . . . . . . . 15

1.3 FKM isoparametric hypersurfaces . . . . . . . . . . . . . . . 20

2 Examples of singular Riemannian foliations of higher codimension $\quad 35$

2.1 Clifford foliations . . . . . . . . . . . . . . . . . 35

2.2 Composed foliations . . . . . . . . . . . . . . . . . . 45

2.3 Bijection between Clifford systems and singular Riemannian foliations . . . 49

3 Homogeneity in Clifford, FKM and composed foliations 53

3.1 Relation between Clifford systems and some Lie groups . . . . . . . . . . . 53

3.2 Homogeneity in Clifford, FKM and composed foliations . . . . . . . . . . 55

$\begin{array}{ll}\text { A Riemannian submersions and submetries } & 63\end{array}$

$\begin{array}{ll}\text { B Riemannian Orbifolds } & 67\end{array}$

$\begin{array}{ll}\text { Bibliography } & 69\end{array}$ 
viii CONTENTS 


\section{Introduction}

Riemannian geometry is a specialization of the theory of smooth manifolds, that allow us studying natural concepts such as distances and angles in those objects. In order to do so, a Riemannian manifold is described, roughly speaking, as a smooth manifold equipped with a Riemannian metric, which makes possible, e.g., to measure and determine how curved is that ambient space or something on it. It is worth mentioning that any smooth manifold admits that type of metric. Riemannian manifolds have a beautiful and rich underlying geometry, hence it remains a very active research field.

An useful method for grasping these objects is decomposing them on simpler pieces of lower dimensions, and keeping some compatibility condition with the original structure; here is when a singular Riemannian foliation (SRF in short) comes out. Although a precise definition will be given in Section 1.1, for now we can say it is a partition $\mathcal{F}$ of a Riemannian manifold $M$ into submanifolds called leaves, not necessarily of the same dimension, which are locally parallel from each other. For instance, the division consisting in all the vertical lines is a SRF, whose leaves have the same dimension, of the plane; while slicing a sphere in circles (1-dimensional leaves) centered in the $z$-axis together with both the north and south poles (0-dimensional leaves) is another example of a SRF.

The core of this document is SRF in spheres, a quite relevant case since those provide an adequate description of more general Riemannian foliations around a point. In particular, we will study two recent classes of the former foliations, named Clifford foliations and composed foliations (the first are included in the second), and some of their properties, due to Radeschi in 2014 [Rad14]. That work is a noteworthy contribution in the categorization of indecomposable non-homogeneous Riemannian foliations of arbitrary codimension in spheres, that remains incomplete. He generalized all the previous examples of Riemannian foliations inspiring his work on the construction of isoparametric hypersurfaces owed to Ferus, Karcher and Münzner [FKM81].

After Radeschi's paper appeared, further progress in singular Riemannian foliations has been made. In 2015, he and Gorodski completed the classification of the homogeneous foliations, produced from Clifford systems in spheres [GR16], which indeed Radeschi had already started. In the same year Lytchak and Radeschi [LR15] proved that every SRF in a sphere can be expressed by a polynomial map. Thus each leaf is a solution set for a system of polynomial equations. This supplies a major bond with algebraic objects that are rather well understood, and enriches the study of Riemannian manifolds from a different 
perspective. That result was refined by Mendes and Radeschi one year later [MR16], as they characterized Clifford foliations in terms of basic polynomials. Another result in this direction was the positive answer to the conjecture of Molino (solved by Alexandrino and Radeschi [AR16]) stating that the partition given by the closures of the leaves of a SRF is again a SRF. Those works will be commented in detail throughout this text, since they are closely related with the topics treated herein.

The content of this dissertation is organized in three chapters, beginning in Chapter 1 with some background material on singular Riemannian foliations, isometric actions on Riemannian manifolds, Clifford theory and isoparametric hypersurfaces. Section 1.1 includes the definition of a singular Riemannian foliation $(M, \mathcal{F})$ as well as some of its key examples, e.g., Riemannian submersions, covering maps and homogeneous foliations. The latter case is a foliation produced by an isometric action of a Lie group $G$ on a Riemannian manifold $M$, and it will be fundamental throughout our text. On the other hand, a foliation that is not of that sort is then denominated non-homogeneous. To understand those group actions and their orbital foliations, the notions of slices, principal orbits and tubular neighborhoods are briefly reviewed. Finishing this section it is explained why the local model of a SRF around a point can be reduced to the study on spheres; for that aim it will be introduced a foremost result, the homothetic lemma transformation, and the notion of infinitesimal foliations whose leaves are of the form

$$
L_{v}:=\left\{w \in \nu_{p} L_{p} \mid \exp _{p} t w \in L_{\exp _{p} t v}\right\}, \quad \text { for a sufficiently small } t>0
$$

This is followed in Section 1.2 by the description and properties of Clifford systems and the Clifford sphere, requiring first some basic concepts on Clifford algebras and Clifford representations. Such algebraic apparatus is essential in the construction of the two families of foliations given by Radeschi in his article. The last part of this chapter (Section 1.3) deals with a family of isoparametric hypersurfaces developed by Ferus, Karcher and Münzner, found in the literature as FKM family. It is important pointing out that most of the theory of isoparametric hypersurfaces is due to Cartan and Münzner. After expressing them analytically by homogeneous and isoparametric functions, the theory is developed into a geometric interpretation through isoparametric hypersurfaces, parallel hypersurfaces, tubes and focal sets, and there are stated some results, e.g., the Cartan-Münzner differential equations in Theorem 1.42 and the FKM family in Theorem 1.43, showing those constructions are just two sides of the same coin. As an application that will be relevant in next chapter, it is proved that any family of isoparametric hypersurfaces in spheres of FKM-type has $g=4$ constant principal curvatures and we compute their specific form. The proofs presented in Theorems 1.37 and 1.40 essentially follow the treatment in [CR15].

Chapter 2 is devoted to the construction of Clifford $\mathcal{F}_{C}$ and composed $\mathcal{F}_{0} \circ \mathcal{F}_{C}$ foliations in the sphere $\mathbb{S}^{2 l-1}[\operatorname{Rad} 14$, Section 2], the main topics of this work. The former are defined 
in Section 2.1 as the fibers of the map

$$
\begin{aligned}
\pi_{C}: \mathbb{S}^{2 l-1} \subseteq \mathbb{R}^{2 l} & \longrightarrow \mathbb{D}_{C} \subseteq \mathbb{R}^{m+1} \\
x & \longmapsto \pi_{C}(x)=\left(\left\langle P_{0} x, x\right\rangle, \ldots,\left\langle P_{m} x, x\right\rangle\right)=\sum_{i=0}^{m}\left\langle P_{i} x, x\right\rangle P_{i},
\end{aligned}
$$

where $\mathbb{D}_{C}$ is the unit disk on $\mathbb{R}^{m+1}$ and the subscript $C$ is related to a Clifford system of rank $m+1$ on $\mathbb{R}^{2 l}$ and $\left(P_{0}, \ldots, P_{m}\right)$ is an orthonormal basis of elements in $C$ for $\mathbb{R}^{m+1}$. Submersion properties and descriptions of its fibers are exhibited in Proposition 2.2. Then it is shown that the fibers of $\pi_{C}$ form a transnormal system (Proposition 2.6), one of the two conditions for a partition to be SRF, which establishes that the leaves must be locally parallel from each other - the second one will be proved in the next section, after defining composed foliations. Further, it is determined that the image of $\pi_{C}$ is the boundary $\mathbb{S}_{C}$ of $\mathbb{D}_{C}$ if $l=m$, or the disk $\mathbb{D}_{C}$ itself, if $l>m+1$, both equipped with a metric of constant curvature 4. An immediate consequence is that $\pi_{C}$ is always a Hopf fibration for the case $l=m$, see Corollary 2.10, as well as the specific situation for $\mathbb{S}^{3}$, where $l=2=m$, in Example 2.9. As we already commented, Clifford foliations are a particular family of composed foliations. As another example, Corollary 2.11 shows how the FKM family discussed throughout Section 1.3 can be recovered from Clifford foliations via the composition $F_{0}=f \circ \pi_{C}$,

$$
\begin{aligned}
F_{0}: \mathbb{S}^{2 l-1} & \stackrel{\pi_{C}}{\longrightarrow} \mathbb{D}_{C} \stackrel{f}{\longrightarrow}[-1,1] \\
x & \longmapsto P \\
\longmapsto & \longmapsto 1-2\|P\|^{2},
\end{aligned}
$$

namely, its leaves are given by the level sets of

$$
F_{0}(x)=1-2 \sin ^{2}(2 t)=\cos (4 t), \quad \text { for } t \in[0, \pi / 4] .
$$

Following Radeschi [Rad14, Section 3], we begin Section 2.2 defining the latter foliations through the fibers of a submetry

$$
\mathbb{S}^{2 l-1} \longrightarrow \triangleq
$$

where $\underline{\Delta}=\frac{1}{2} \Delta$ or $\frac{1}{2}(\Delta \star\{p t\})$, depending on whether the image of $\pi_{C}$ is $\mathbb{S}_{C}$ or $\mathbb{D}_{C}$; here the leaf space is denoted by $\underline{\Delta}$, the operation $\star$ represents the spherical join and the $\frac{1}{2}$ factor indicates a rescaling of the metric. Subsequently, it is proved in Propositions 2.13 to 2.15 that composed foliations are SRF. Hence the remaining characteristic to be verified for Clifford foliations, i.e, to be a Riemannian foliation, is obtained in Corollary 2.16 as a consequence of them being special case of composed foliations. Section 2.3 unveils a stunning correspondence between Clifford systems (algebraic objects) and singular Riemannian foliations on spheres (geometric objects). Even though the isoparametric families in [FKM81] were built up from Clifford systems, there is not a bijection between them. In other words, a FKM foliation does not necessarily come from a Clifford system, whose quotient is isometric to a FKM example; but if so, there could be distinct (inequivalent) Clifford systems giving rise to the same FKM foliation. Nevertheless, Radeschi [Rad14, Section 4] obtained a powerful algebraic-geometric connection in virtue of his two types of foliations. Namely, if $\mathfrak{C}$ is the 
class of Clifford systems and $\mathfrak{F}$ is the class of SRF whose quotient is a sphere or a hemisphere of constant curvature 4 , there is a bijection map,

$$
\begin{aligned}
\mathfrak{C} /\{\text { geometric equivalence }\} & \longrightarrow \mathfrak{F} /\{\text { congruence }\} \\
C & \longmapsto \mathcal{F}_{C},
\end{aligned}
$$

assigning to each Clifford system $C$ a unique Clifford foliation $\mathcal{F}_{C}$. This result is tackled in Propositions 2.18 and 2.19; the first establishes that every singular Riemannian foliation on a sphere whose quotient is a sphere or a hemisphere of curvature 4 is indeed a Clifford foliation; while the second shows that inequivalent Clifford systems distinguish incongruent Clifford foliations.

Chapter 3 mainly investigates homogeneity properties involving FKM, Clifford and composed foliations. Before we get down to it, Section 3.1 examines two special symmetry properties of Clifford foliations and their relation to some Lie groups: on one hand, every element $P \in \mathbb{S}_{C}$ is an orthogonal map - seen as a foliated isometry induced by a Pin subgroup of $\mathrm{O}(2 l)$ - on the sphere $\mathbb{S}^{2 l-1}$ equipped with a Clifford foliation $\mathcal{F}_{C}$; on the other hand, the action of $P$, when it descends through $\pi_{C}: \mathbb{S}^{2 l-1} \rightarrow \mathbb{D}_{C}$, produces a reflection along the segment through $P$-which can be seen as an isometric action of a Spin subgroup of $\mathrm{SO}(2 l)$ - . Once the machinery is set up, Section 3.2 begins explaining that the only two cases which a Clifford foliation of $\mathbb{S}^{2 l-1}$ whose quotient is a sphere $\mathbb{S}^{m}$ is homogeneous are for $m=2$ or 4 . Namely, all the Clifford foliations in $\mathbb{S}^{2 l-1}$ are non-homogeneous, but three cases detailed in Proposition 3.6. We also highlight an example of a non-homogeneous foliation on $\mathbb{R}^{31}$ constructed from a Clifford system of rank 10, see Proposition 3.7. The remaining results, discuss relations among the three aforementioned classes of foliations.

Finally, we decided to include two appendices, covering notions used in some proofs in the main text. The purpose of Appendix A is completing the digression of submetries and Riemannian submersions used in the construction of composed foliations (Section 2.2). There are proved some properties of submetries that, though commonly mentioned in SRF's theory, it is hard finding proofs written in books and papers. Appendix B is a brief compilation of basic definitions and examples in Riemannian orbifolds, that were used in Proposition 3.7. 


\section{Chapter 1}

\section{Algebraic and geometric preliminaries}

This chapter is devoted to the basic apparatus needed for our study of singular Riemannian foliations in spheres. With this in mind we begin defining a singular Riemannian foliation in Section 1.1, we give some important examples and list some properties as well. In Section 1.2 we provide the fundamentals of Clifford theory such as Clifford algebras, their representations and systems, and we finish this study with the relation between the last two, which will be essential in the construction of the FKM examples. Finishing our study we develop in Section 1.3 part of the theory of isoparametric hypersurfaces, focusing most in the real space form $\mathbb{S}^{n}$, that is the context worked by Marco Rasdechi in [Rad14]. In this way we mainly review the concepts of a real space form, focal manifolds, tubes and parallel hypersurfaces and prove some important theorems about principal curvatures. We use as references two books of Cecil and, of course, the paper of Radeschi mentioned in the introduction.

\subsection{Singular Riemannian foliations}

Definition 1.1. Let $M$ be a complete Riemannian manifold, and $\mathcal{F}$ a partition of $M$ into complete, connected, injectively immersed manifolds, called leaves. The pair $(M, \mathcal{F})$ is called:

- a singular foliation if there is a family of smooth vector fields $\left\{X_{i}\right\}$ that span the tangent space of the leaves at each point.

- a transnormal system if any geodesic starting perpendicular to a leaf, stays perpendicular to all the leaves it meets. Such geodesics are called horizontal geodesics.

- a singular Riemannian foliation if it is both a singular foliation and a transnormal system.

Equivalently, a singular foliation could be described as a foliation whose leaves do not need to have the same dimension. While if they have the same dimension, it is called a regular foliation. There exists another characterization for a transnormal system, to say, the leaves are locally equidistant, and henceforth we will use both descriptions indistinctly. 
Remark 1.2. We also want to underline a conjecture which arises from the previous definition. There is an educated guess that if $(M, \mathcal{F})$ is a transnormal system then it is automatically a (singular) foliation.

Before giving some examples of singular Riemannian foliations (SRF), let us present some of their properties as well as give two non-examples, namely, where transnormality and singular foliation conditions fail.

Throughout this section we will assume any smooth manifold to be complete and Riemannian, and $(M, \mathcal{F})$ will denote a singular Riemannian foliation. Given $(M, \mathcal{F})$, the space of leaves, denoted by $M / \mathcal{F}$, is the set of leaves of $\mathcal{F}$ endowed with the topology induced by the canonical projection $\pi: M \rightarrow M / \mathcal{F}$ that sends a point $p \in M$ to the leaf $L_{p} \in \mathcal{F}$ containing it. We now proceed to define a stratification on $(M, \mathcal{F})$.

Definition 1.3 (Stratification). For each nonnegative integer $r$ define $\sum_{r}$ to be the union of leaves of dimension $r$. The connected components of each $\sum_{r}$ are (possibly incomplete) submanifolds and are called strata of $(M, \mathcal{F})$. We denote by $\operatorname{dim} \mathcal{F}$ the maximum dimension of the leaves in $\mathcal{F}$, and call regular leaf one having maximal dimension, and a point belonging to it a regular point. The set of regular leaves $\sum_{\operatorname{dim} \mathcal{F}}$ is open, dense and connected, and therefore it defines a stratum which we call the regular stratum. Finally, $(M, \mathcal{F})$ is closed if all the leaves of $\mathcal{F}$ are closed. In this case the leaves are at a constant distance from each other, and the space of leaves $M / \mathcal{F}$ has the structure of a Hausdorff metric space.

Example 1.4 (Non-examples of singular Riemannian foliations).

- Not a transnormal system: consider a (singular) foliation on $\mathbb{R}^{2}$ given by the two families of hyperbolae $H_{1}:=\left\{(x, y) \in \mathbb{R}^{2}: x^{2}-y^{2}=d, d \in \mathbb{R}_{+}\right\}, H_{2}:=\left\{(x, y):-x^{2}+\right.$ $\left.y^{2}=d, d \in \mathbb{R}_{+}\right\}$together with the four rays $\left\{(x, y) \in \mathbb{R}^{2}: x^{2}-y^{2}=0,(x, y) \neq(0,0)\right\}$ and the origin, the latter being the leaf with a different dimension, as illustrated in Figure 1.1. Now, since the geodesics in $\mathbb{R}^{2}$ are the straight lines, let $L$ be a line emanating from the origin and passing through the point $(a, b)$ in the hyperbola $x^{2}-y^{2}=d$, such that $a \neq 0$ and $b \neq 0$. Then $L$ must be of the form

$$
y=\frac{b}{a} x
$$

The tangent line to $x^{2}-y^{2}=d$ at $(a, b)$ has slope $m=\frac{a}{b}$. Thus the normal line at that point has slope $\frac{-1}{m}=\frac{-b}{a}$. Therefore $L$ crosses the leaf $x^{2}-y^{2}=d$ but not orthogonally, since $\frac{b}{a} \neq \frac{-b}{a}$.

Alternatively, from the characterization of leaves remaining at constant distance from each other, is easy to see that if a single point $p$ is a leaf, there can only exists two kind of foliations in $\mathbb{R}^{2}$ : the one where each point in $\mathbb{R}^{2}$ is a single leaf, we will call it the finest foliation; or the one where the leaves are given by the concentric circles around $p$, e.g., see Figure 1.2.

- Not a singular foliation: In the Euclidean plane $\mathbb{R}^{2}$, consider $L$ to be the following 


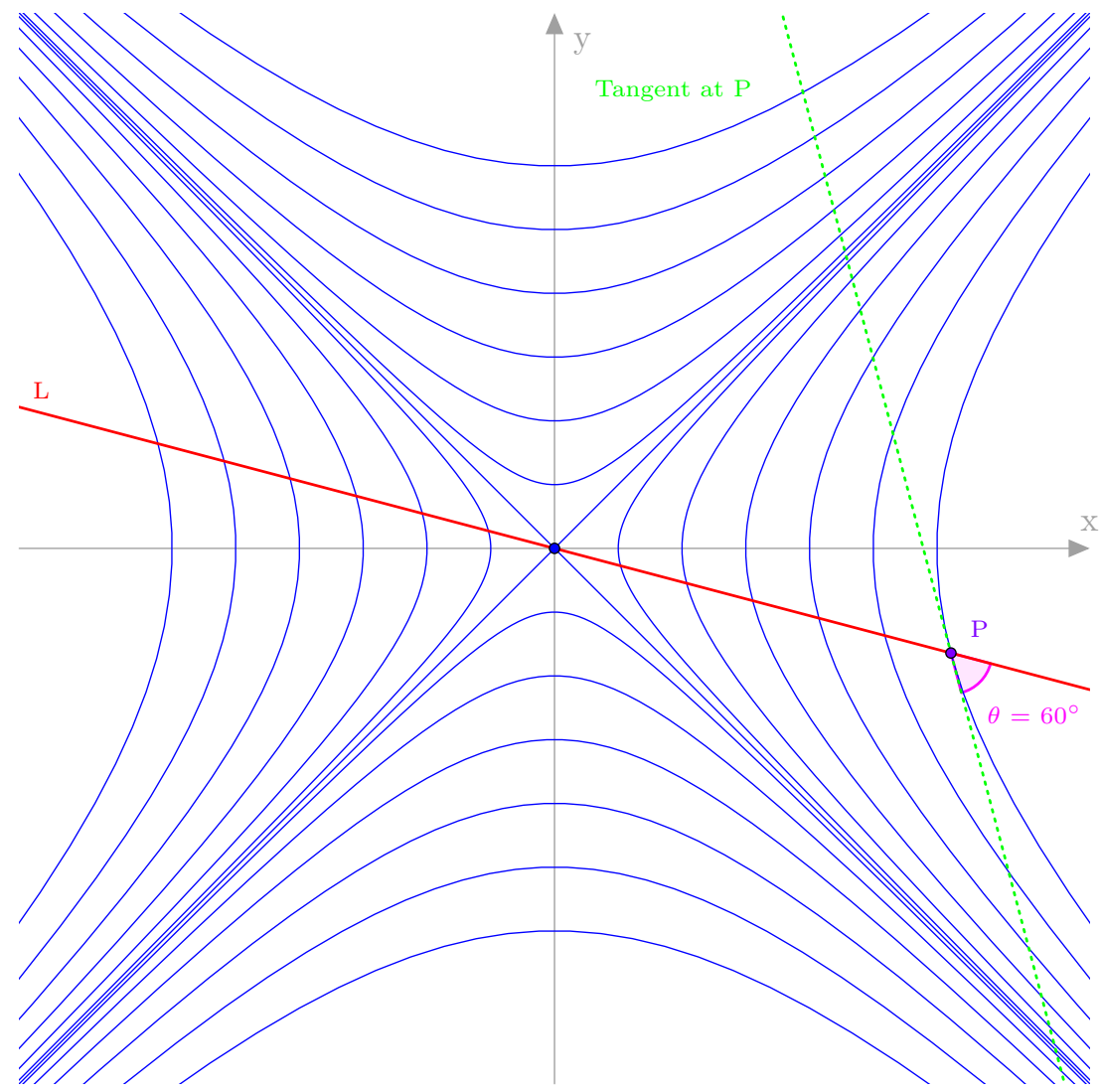

Figure 1.1: The geodesic $L$ does not meet orthogonally this singular foliation of $\mathbb{R}^{2}$ at the point p.

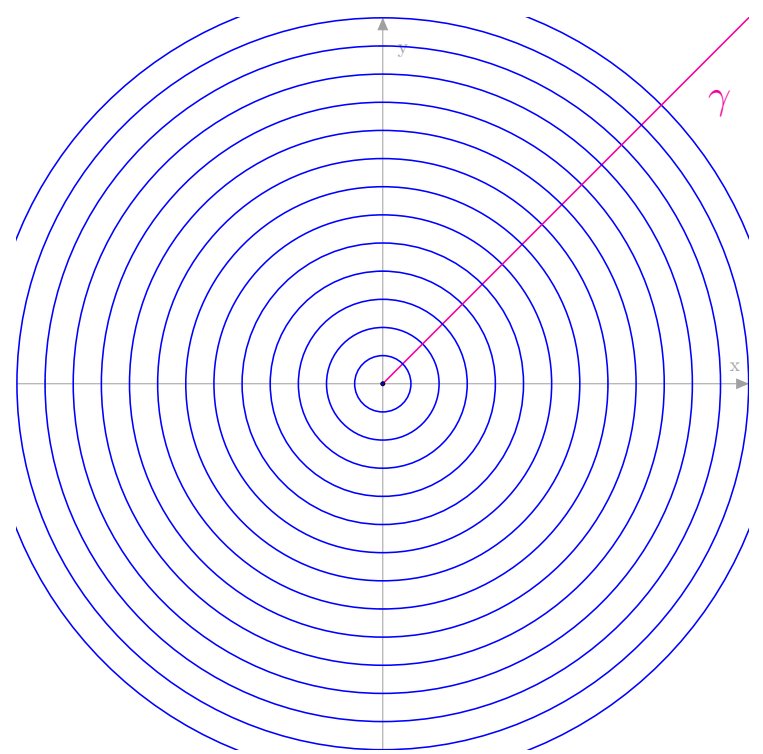

Figure 1.2: $S R F$ of concentric circles around a point in $\mathbb{R}^{2}$. The geodesic $\gamma$ meets each leaf orthogonally.

set of straight lines

$$
L:=\bigcup_{b \geqslant 0}\{(x, y) \mid y=x+b\}
$$

and $P$ to be the set of points given by

$$
P:=\bigcup_{y \leqslant x}\{(x, y)\}
$$


The partition $L \cup P$ of $\mathbb{R}^{2}$ (see Figure 1.3a) is neither a foliation nor a transnormal system. On one hand, the leaves in $P$ have vector field zero, whereas the ones in $L$ should have a nowhere-vanishing smooth vector field. Therefore, the continuity of a vector field for the partition above fails when approaching to the leaf $y=x$ from the right hand side. Thus $L \cup P$ is not a foliation of $\mathbb{R}^{2}$. On the other hand, any straight line emanating from a point $(x, y) \in P$ and having slope $m \neq-1$ does meet each leaf in $L$ but not orthogonally; then the partition $L \cup P$ is not a transnormal system.

Another example of partition in $\mathbb{R}^{2}$ which fails to be a foliation is shown in Figure 1.3b. Notice again that a vector field spanning its leaves cannot be continuous, as in the case above.

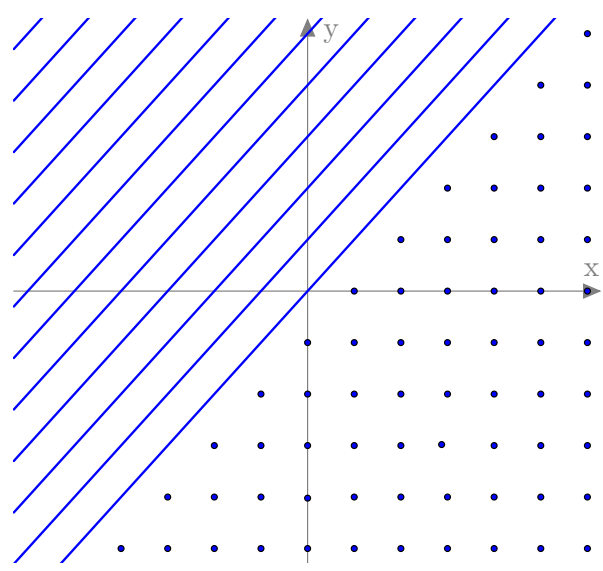

(a)

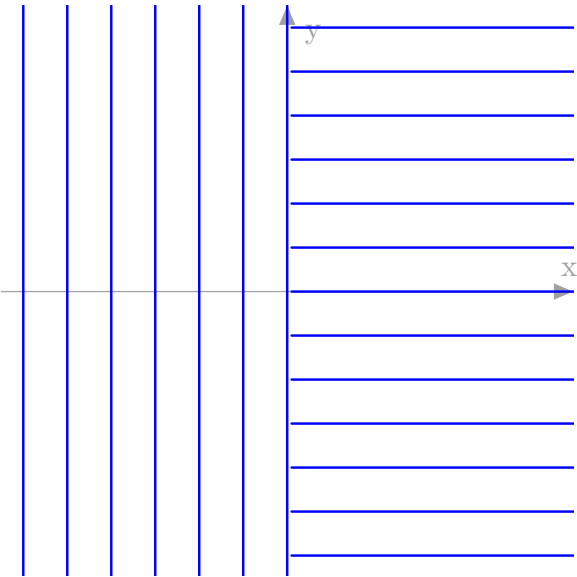

(b)

Figure 1.3: These partitions of $\mathbb{R}^{2}$ are not foliations, nor are transnormal systems.

\section{Examples of singular Riemannian foliations}

Example 1.5 (Trivial foliations).

1. Consider the finest foliation given by the points of $M$, which we denote $(M,\{p t s\})$. This is clearly a singular Riemannian foliation and it is the only one with $\operatorname{dim} \mathcal{F}=0$.

2. In contrast, take the coarsest foliation with one leaf corresponding to the whole M, which we denote $(M, M)$. This is the only SRF such that $\operatorname{codim} \mathcal{F}=0$.

Example 1.6 (Simple foliation). If $M, N$ are Riemannian manifolds and $\pi: M \rightarrow N$ is a Riemannian submersion, the connected components of the partition $\mathcal{F}$ on $M$, defined by the fibers of $\pi$, are embedded submanifolds, due to the local form of a submersion, and are also complete since $M$ is complete. This gives us a foliation whose leaves have the same dimension and thus it is regular (in consequence, singular). Last, it is a transnormal system by [GHL12, Proposition 2.109]. We call this singular Riemannian foliation a simple foliation.

Concerning this example, we want to highlight that every regular Riemannian foliation is modeled locally by a Riemannian submersion. 
Example 1.7 (Pullback foliation in a Riemannian submersion). Let $\pi: M \rightarrow N$ a Riemannian submersion, such that $(N, \mathcal{F})$ is a SRF. It is possible to define a foliation $\tilde{\mathcal{F}}$ in $M$ by pulling back $\mathcal{F}$. The leaves of $\tilde{\mathcal{F}}$ are given by the (connected components of) preimages of leaves:

$$
\tilde{\mathcal{F}}:=\pi^{*} \mathcal{F}=\left\{\tilde{L}=\pi^{-1}(L) \mid L \in \mathcal{F}\right\} .
$$

Since $\pi$ is a Riemannian submersion, then tangent space to the leave $\tilde{L}_{p}$ at a point $p \in M$ splits as

$$
T_{p} L_{p}=\mathcal{V}_{p} \oplus \widetilde{T_{\pi(p)} L_{\pi(p)}},
$$

where $T_{\pi(p)} L_{\pi(p)}$ is the horizontal lift of $T_{\pi(p)} L_{\pi(p)}$. Again, [GHL12, Proposition 2.109] guarantees that a geodesic in $M$ starting perpendicular to a leaf $\tilde{L}$ corresponds to a horizontal lift $\tilde{\gamma(t)}$ of a geodesic $\gamma(t)$ in $N$. Moreover, for every $t, \gamma^{\prime}(t) \perp \mathcal{V}_{\gamma(t)}$, since $\tilde{\gamma(t)}$ is horizontal, and $\gamma^{\prime}(t) \perp \widetilde{T_{\pi(p)} L_{\pi(p)}}$ as well because $\gamma^{\prime}(t)$ is perpendicular to $T_{\pi(p)} L_{\pi(p)}$ in $N$. This shows that the foliation $\tilde{\mathcal{F}}=\pi^{*} \mathcal{F}$ is a transnormal system, whence, a SRF of $M$.

Example 1.8 (Homogeneous foliation). If $G$ is a connected Lie group acting on $M$ by isometries, the foliation $\mathcal{F}$ on $M$ defined by the orbits of $G$ is a singular Riemannian foliation denominated homogeneous foliation. More precisely, for every $X \in \mathfrak{g}$, there is a corresponding action field, called the fundamental vector field $X^{\#}$ on $M$, and they generate $\mathfrak{X}(\mathcal{F})$. At the same time they are Killing vector fields, since $M$ is complete, which implies $\mathcal{L}_{X \#} g=0$, and in particular for the perpendicular (horizontal) part of the metric we also have $\mathcal{L}_{X \#} g^{T}=0$. For any $X=\sum f_{i} X_{i}^{\#} \in \mathfrak{X}(\mathcal{F})$, with $f_{i}$ some smooth functions,

$$
\begin{aligned}
\mathcal{L}_{X} g^{T}(Y, Z)= & \sum \mathcal{L}_{f_{i} X_{i}^{\#}} g^{T}(Y, Z) \\
= & \sum f_{i} X_{i}^{\#} g^{T}(Y, Z)-g^{T}\left(\left[f_{i} X_{i}^{\#}, Y\right], Z\right)-g^{T}\left(Y,\left[f_{i} X_{i}^{\#}, Z\right]\right) \\
= & \sum f_{i} X_{i}^{\#} g^{T}(Y, Z)-g^{T}\left(\nabla_{f_{i} X_{i}^{\#}} Y-\nabla_{Y}\left(f_{i} X_{i}^{\#}\right), Z\right) \\
& -g^{T}\left(Y, \nabla_{f_{i} X_{i}^{\#}} Z-\nabla_{Z}\left(f_{i} X_{i}^{\#}\right)\right) \\
= & \sum f_{i} X_{i}^{\#} g^{T}(Y, Z)-g^{T}\left(\nabla_{f_{i} X_{i}^{\#}} Y, Z\right)-g^{T}\left(Y, \nabla_{f_{i} X_{i}^{\#}} Z\right) \\
& +g^{T}\left(\nabla_{Y}\left(f_{i} X_{i}^{\#}\right), Z\right)+g^{T}\left(Y, \nabla_{Z}\left(f_{i} X_{i}^{\#}\right)\right) \\
= & \sum f_{i} X_{i}^{\#} g^{T}(Y, Z)-f_{i} g^{T}\left(\nabla_{X_{i}^{\#}} Y, Z\right)-f_{i} g^{T}\left(Y, \nabla_{X_{i}^{\#}} Z\right) \\
& +Y\left(f_{i}\right) g^{T}\left(X_{i}^{\#}, Z\right)+Z\left(f_{i}\right) g^{T}\left(Y, X_{i}^{\#}\right) \\
& +f_{i} g^{T}\left(\nabla_{Y} X_{i}^{\#}, Z\right)+f_{i} g^{T}\left(Y, \nabla_{Z} X_{i}^{\#}\right) .
\end{aligned}
$$

The sum of the last two terms in the last line is zero because $X^{\#}$ is Killing field. The first and second terms in the same line also vanish as $X_{i}^{\#}$ is vertical and $g^{T}\left(X_{i}^{\#}, \cdot\right)=0$. Moreover, using that the Levi-Civita connection has no torsion, we get

$$
\begin{aligned}
\mathcal{L}_{X} g^{T}(Y, Z)= & \sum f_{i} X_{i}^{\#} g^{T}(Y, Z)-f_{i} g^{T}\left(\nabla_{Y} X_{i}^{\#}, Z\right)-f_{i} g^{T}\left(\left[X_{i}^{\#}, Y\right], Z\right) \\
& -f_{i} g^{T}\left(Y, \nabla_{Z} X_{i}^{\#}\right)-f_{i} g^{T}\left(Y,\left[X_{i}^{\#}, Z\right]\right) \\
= & 0,
\end{aligned}
$$


using again that $X^{\#}$ is vector Killing field. From [MC88, Section 3.2], if $\mathcal{L}_{X} g^{T}(Y, Z)=0$, then the horizontal space is preserved under the geodesic flow, and therefore the transnormal system condition is satisfied.

Explicit examples of isometric group actions could be, for instance:

- the circle $\mathbb{S}^{1} \cong \mathrm{SO}(2)$ acting on $\mathbb{S}^{2}$ by rotation about the $z$-axis, where the North and South poles together with all the circles around that axis form the leaves of this foliation.

- the Hopf action $\mathrm{U}(1) \cong \mathbb{S}^{1} \subseteq \mathbb{C}$ on $\mathrm{SU}(2) \cong \mathbb{S}^{3} \subseteq \mathbb{C}^{2}$ by right group action

$$
e^{i \theta} \cdot\left[\begin{array}{cc}
u & \bar{v} \\
-\bar{v} & u
\end{array}\right] \longmapsto e^{i \theta} \cdot(u, v)=: x \in \mathbb{S}^{3},
$$

where $u, v \in \mathbb{C}$ and $|u|^{2}+|v|^{2}=1$. This action can also be seen as

$$
\left[\begin{array}{cc}
z & 0 \\
0 & z
\end{array}\right] \cdot\left[\begin{array}{cc}
u & v \\
-\bar{v} & \bar{u}
\end{array}\right] \longmapsto\left[\begin{array}{cc}
z u & z v \\
-\overline{z v} & \overline{z u}
\end{array}\right] \in \mathrm{SU}(2)
$$

where $z:=e^{i \theta}$. The leaf space is $\mathbb{S}^{3} / \mathbb{S}^{1} \cong \mathbb{S}^{2}$ and each fiber is a circle in $\mathbb{S}^{3}$, which is called the Hopf foliation.

Example 1.9 (Covering maps).

1. If $(\tilde{M}, \tilde{\mathcal{F}})$ is a singular Riemannian foliation and $G$ is a group acting freely, properly discontinuous and isometrically on $\tilde{M}$ by the $G$-maps

$$
\begin{aligned}
\mu: G \times \tilde{M} & \longrightarrow \tilde{M} \\
(g, \tilde{p}) & \longmapsto \mu(g, \tilde{p}):=g \cdot \tilde{p}=g p
\end{aligned}
$$

that sends leaves to leaves, then $\tilde{M} / G=: M$ inherits a singular Riemannian foliation $\tilde{\mathcal{F}} / G=: \mathcal{F}$. To see this, let $\mathcal{F}:=\left\{L_{p}=\pi\left(\tilde{L}_{\tilde{p}}\right) \mid \pi(\tilde{p})=p\right\}$. This foliation is well defined for if $\pi(\tilde{p})=\pi(\tilde{q})=p$, then there exists $g \in G$ such that the map

$$
\begin{aligned}
\mu_{g}(\tilde{p}): \tilde{M} & \longrightarrow \tilde{M} \\
\tilde{p} & \longmapsto \mu_{g}(\tilde{p}):=\mu(g, \tilde{p})
\end{aligned}
$$

evaluated at $p$ gives $\mu_{g}(\tilde{p})=\tilde{q}$ and thus $\mu_{g}\left(\tilde{L}_{\tilde{p}}\right)=\tilde{L}_{\tilde{q}}$. Also $\mathcal{F}$ is singular: since $\pi$ is a submersion, for any vector $v \in T_{\gamma(t)} L$ there exists a vector $\tilde{v} \in T_{\tilde{p}} \tilde{L}_{\tilde{p}}$ such that $d \pi(\tilde{v})=v$ and $\pi(\tilde{p})=p$, and using the fact of $\tilde{\mathcal{F}}$ being singular and for all $\tilde{p}$ in $\tilde{M}$, $d \pi\left(T_{\tilde{p}} \tilde{L}_{\tilde{p}}\right)=T_{p} L_{p}$, we can define a smooth vector field $X \in \mathfrak{X}(\mathcal{F})$ where $X_{p}=v$. To show the foliation in the quotient is transnormal, take a geodesic $\gamma$ in $\tilde{M} / G$ starting orthogonal to a leaf, let $\tilde{\gamma}$ be its horizontal lift and decompose any $\tilde{v}$ in $T_{\tilde{p}} \tilde{L}_{\tilde{p}}$ as

$$
\tilde{v}=\tilde{v}_{1}+\tilde{v}_{2}, \quad \tilde{v}_{1} \in T_{\tilde{p}} \pi^{-1}(p), \quad \tilde{v}_{2} \in T_{\tilde{p}} \tilde{L}_{\tilde{p}} \cap\left(T_{\tilde{p}} \pi^{-1}(p)\right)^{\perp} .
$$


Since $\pi$ is a local isometry,

$$
\begin{aligned}
g\left(\gamma^{\prime}, v\right) & =\tilde{g}\left(\tilde{\gamma}^{\prime}, \tilde{v}\right) \\
& =\tilde{g}\left(\tilde{\gamma}^{\prime}, \tilde{v}_{1}\right)+\tilde{g}\left(\tilde{\gamma}^{\prime}, \tilde{v}_{2}\right) \\
& =g\left(\gamma^{\prime}, d \pi\left(\tilde{v}_{2}\right)\right) \\
& =0
\end{aligned}
$$

where in the second line the first term vanishes since $\tilde{\gamma}$ is horizontal lift and $\pi$ a submersion, and the next to last line is zero since for all $\tilde{p}$ in $\tilde{M}, d \pi\left(T_{\tilde{p}} \tilde{L}_{\tilde{p}}\right)=T_{p} L_{p}$, and $d \pi$ is an isometry on the horizontal space.

Let $\tilde{M}:=\mathbb{S}^{n}$ with the foliation $\tilde{\mathcal{F}}$ given by the latitude circles with respect to the $x_{n+1}$-axis and the two poles and $G:=\{i d,-i d\}$ be a particular example of this case. Thus $\mathbb{R} P^{n}$ is the resulting quotient and its foliation consists in the upper half circles of the $\mathbb{S}^{n}$ foliation and a single point.

2. If $(M, \mathcal{F})$ is a singular Riemannian foliation and $\pi: \tilde{M} \mapsto M$ is a covering map, then the preimages of the leaves of $\mathcal{F}$ define a singular Riemannian foliation $\tilde{\mathcal{F}}$ on $\tilde{M}$. Define $\tilde{\mathcal{F}}:=\left\{\pi^{-1}(\mathcal{F})\right\}$. Observe that the points $\mathcal{F}^{\pi}:=\left\{L_{\tilde{p}}^{\pi}=\pi^{-1}(p) \mid \pi(\tilde{p})=p, p \in M\right\}$ form a singular Riemannian foliation and $L_{\tilde{p}}^{\pi} \subseteq \tilde{L}_{\tilde{p}}$ for each $\tilde{p}$ in $\tilde{M}$ and $\tilde{L}_{\tilde{p}} \in \tilde{\mathcal{F}}$. Take $\tilde{\gamma}$ orthogonal to a leaf in $\tilde{M}$. In particular it is orthogonal to $\mathcal{F}^{\pi}$ and $\pi \circ \tilde{\gamma}=\gamma$ is orthogonal to $\mathcal{F}$. Using that $T_{\tilde{p}} L_{\tilde{p}}^{\pi}$ is zero and $\pi$ is a local isometry, we deduce by a similar argument as in item 1 . that

$$
\tilde{g}\left(\tilde{\gamma}^{\prime}, v\right)=0, \quad \text { for all } v \in T_{\tilde{p}} \tilde{L}_{\tilde{p}}
$$

To illustrate this, we claim that any singular Riemannian foliation in the torus $T^{2}$ must have all their leaves of the same dimension. Otherwise, suppose there exists a leaf with a different dimension. The universal covering of $T^{2}$ is $\mathbb{R}^{2}$ in which the only singular (non regular) Riemannian foliation is a point and concentric circles around it. However this foliation is not invariant under the quotient, whence, the initial foliation in $T^{2}$ should be regular.

Example 1.10 (Products). For $(M, \mathcal{F}),\left(M^{\prime}, \mathcal{F}^{\prime}\right)$ singular Riemannian foliations, we can consider the product manifold $M \times M^{\prime}$ with the product metric, where we have the product foliation $\mathcal{F} \times \mathcal{F}^{\prime}$ such that the leaf passing through $\left(p, p^{\prime}\right)$ is given by $L_{p} \times L_{p^{\prime}}$. Since $\mathcal{F}$ and $\mathcal{F}^{\prime}$ are singular foliations, for any vector $v:=v_{p}+v_{p^{\prime}}$ on $\mathfrak{X}\left(\mathcal{F} \times \mathcal{F}^{\prime}\right)$, there exist vector fields $X \in \mathfrak{X}(\mathcal{F}), X^{\prime} \in \mathfrak{X}\left(\mathcal{F}^{\prime}\right)$ such that $X_{p}=v_{p}, X_{p^{\prime}}=v_{p^{\prime}}$. Then the vector field $Y:=X \oplus X^{\prime}$ belongs $\mathfrak{X}\left(\mathcal{F} \times \mathcal{F}^{\prime}\right)$. Besides, every geodesic on this product manifold can be expressed as $\gamma=\left(\gamma_{M}, \gamma_{M^{\prime}}\right)$, where $\gamma_{M}, \gamma_{M^{\prime}}$ are geodesics on $M, M^{\prime}$, respectively. If $\gamma$ is orthogonal to a leave $L_{p} \times L_{p^{\prime}}$, then $\gamma_{M}, \gamma_{M^{\prime}}$ are orthogonal to $L_{p}, L_{p^{\prime}}$. Using again that $(M, \mathcal{F})$ and $\left(M^{\prime}, \mathcal{F}^{\prime}\right)$ are transnormal systems, the latter geodesics remains orthogonal to all the leaves they cross, whence, $\gamma$ have the same property and the product $\left(M \times M^{\prime}, \mathcal{F} \times \mathcal{F}^{\prime}\right)$ is transnormal.

Take $\left(\mathbb{S}^{n} \times \mathbb{R}, \mathcal{F}_{\text {rot }} \times\{p t s\}\right)$ as an example of product of singular Riemannian foliations, where $\mathcal{F}_{\text {rot }}$ is the foliation given by rotation around an axis, say, $x_{n+1}$. 
Example 1.11 (Suspension of homomorphism). This example combines several of the past constructions. Let $B$ and $T$ be complete connected smooth manifolds of dimensions $k$ and $l$ respectively, and let

$$
\rho: \pi_{1}(B) \rightarrow \operatorname{Isom}\left(T, \mathcal{F}_{T}\right)
$$

be a homomorphism from the fundamental group of $B$ to the group of isometries of $\left(T, \mathcal{F}_{T}\right)$. Define $\tilde{M}:=T \times \tilde{B}$, where $\tilde{B}$ is the universal covering of $B$. The projection on the first coordinate $p r_{1}: T \times \tilde{B} \rightarrow T$ induces a foliation $\tilde{\mathcal{F}}$ on $\tilde{M}$ of codimension $l$ given by its fibers. Additionally, consider the smooth action

$$
\begin{aligned}
\mu: \pi_{1}(B) & \hookrightarrow T \times \tilde{B} \\
{[g] } & \mapsto \mu_{[g]}(t, \tilde{b}):=\left(\rho\left([g]^{-1}\right)(t), \tilde{b} \cdot[g]\right) .
\end{aligned}
$$

Observe this action sends leaves $\tilde{\mathcal{F}}$ to leaves of $\tilde{\mathcal{F}}$ since in the first component every leaf is a point and in the second coordinate there is just one leaf, which is $\tilde{B}$, and thus they are trivially preserved. We say the manifold $M:=\tilde{M} / \pi_{1}(B)$ together with a codimension- $l$ foliation $\mathcal{F}:=\tilde{\mathcal{F}} / \pi_{1}(B)$ is a singular Riemannian foliation constructed by suspension of the homomorphism $\rho$.

For instance, consider $\left(T, \mathcal{F}_{T}\right):=\left(\mathbb{S}^{2}, \mathcal{F}_{\text {rot }}\right)$ to be the sphere with the foliation given by the rotation around the $z$-axis, and let $B:=\mathbb{S}^{1}$ be the unit circle. The universal covering of the unit circle is $\tilde{B}=\mathbb{R}$ and the fundamental group of $B$ is $\pi_{1}(B)=\mathbb{Z}$, which represents a translation in of a point in $\mathbb{R}$ by an integer multiple of $2 \pi$. The product manifold $\tilde{M}=\mathbb{S}^{2} \times \mathbb{R}$ is equipped with the codimension-two foliation

$$
\tilde{\mathcal{F}}=\left\{q \times \mathbb{R}=p r_{1}^{-1}(q) \mid q \in \mathbb{S}^{2}\right\}
$$

Since a homomorphism $\rho: \pi_{1}\left(\mathbb{S}^{1}\right) \rightarrow \operatorname{Isom}\left(\mathbb{S}^{2}, \mathcal{F}_{\text {rot }_{z}}\right)$ should send leaves of $\mathcal{F}_{\text {rot }}$ to leaves of $\mathcal{F}_{\text {rot } z}$, each leaf $L$ in $\mathcal{F}_{\text {rot }_{z}}$ is sent to itself, that is, $\rho\left([g]^{-1}\right)(L)=L$, for every $[g] \in \pi_{1}\left(\mathbb{S}^{1}\right)$. The quotient given by the orbits of the $\pi_{1}\left(\mathbb{S}^{1}\right)$-action on $\mathbb{S}^{2} \times \mathbb{R}$ under the map $\mu$ is

$$
M=\left(\mathbb{S}^{2} \times \mathbb{R}\right) / \mathbb{Z}=\mathbb{S}^{2} \times(\mathbb{R} / \mathbb{Z})=\mathbb{S}^{2} \times \mathbb{S}^{1}
$$

The singular Riemannian foliation constructed by suspension of the homomorphism $\rho$ is the pair $\left(\mathbb{S}^{2} \times \mathbb{S}^{1}, \mathcal{F}\right)$, where $\mathcal{F}$ is the foliation of codimension 2 defined by the images of the leaves in $\tilde{\mathcal{F}}$ under the quotient map $(\tilde{M}, \tilde{\mathcal{F}}) \rightarrow(M, \mathcal{F})$,

$$
\mathcal{F}=\left\{q \times \mathbb{S}^{1} \mid q \in \mathbb{S}^{2}\right\}
$$

\section{Isometric actions and local model of a singular Riemannian foliation}

This section begins providing some definitions on isometric actions that will be specially useful when discussing the relation among Clifford systems, Lie groups and homogeneity properties of Clifford foliations in Chapter 3. Then we will understand that the local structure of a SRF at a point behaves as a SRF of a sphere, and hence the importance of studying the latter model. The homothetic transformation (Lemma 1.20) will be crucial for that purpose and for the construction of composed foliations in Section 2.2. For a closer 
look into all these matters, see [AB15, Chapter 3] and [MC88, Chapter 6].

Definition 1.12. Consider two (left) $G$-actions $\mu_{1}: G \times M_{1} \rightarrow M_{1}$ and $\mu_{2}: G \times M_{2} \rightarrow M_{2}$. A map $f: M_{1} \rightarrow M_{2}$ is called $G$-equivariant if the actions commute with the map, i.e., $\mu_{2}(g, f(p))=f\left(\mu_{1}(g, p)\right)$ for all $p \in M_{1}$ and $g \in G$.

Let $G$ a left action on $M$. In order to avoid confusion of notation, we use $G(p)$ to denote the orbit of $p$ generated by $G$ and $G_{p}$ to denote the isotropy group of $p$.

Definition 1.13. Let $\mu: G \times M \rightarrow M$ an action. A slice at $p \in M$ is an embedded submanifold $S_{p}$ containing $p$ and satisfying the following properties:

(i) $T_{p} M=d \mu_{p} \mathfrak{g} \oplus T_{p} S_{p}$ and $T_{q} M=d \mu_{q} \mathfrak{g}+T_{q} S_{p}$, for all $x \in S_{p}$;

(ii) $S_{p}$ is invariant under $G_{p}$, i.e., if $q \in S_{p}$ and $g \in G_{p}$, then $\mu(g, q) \in S_{p}$;

(iii) If $q \in S_{p}$ and $g \in G$ are such that $\mu(g, q) \in S_{p}$, then $g \in G_{p}$.

Theorem 1.14. If $\mu: G \times M \rightarrow M$ is a proper action, then, for every $p \in M$, there exists a slice $S_{p}$ at $p$.

Remark 1.15. If the action is isometric, such a slice at $p$ is given by

$$
S_{p}=\exp _{p}\left(B_{\varepsilon}(0)\right)
$$

where $B_{\varepsilon}(0)$ is an open ball of a sufficiently small radius $\varepsilon>0$ around the origin in the normal space $\nu_{p} G(p)$.

Definition 1.16. Let $\mu: G \times M \rightarrow M$ be a proper action. Then $G(p)$ is a principal orbit if there exists a neighborhood $U$ of $p$ in $M$ such that for every point $q$ belonging to $U$, $G_{p} \subseteq G_{\mu(g, q)}$, for some $g \in G$.

Proposition 1.17. Let $\mu$ be a proper left $G$-action. Then $G(p)$ is a principal orbit if and only if $G_{p}=G_{q}$, for all $q$ lying in a slice $S_{p}$ at $p$.

Definition 1.18. Let $\mu$ be a proper $G$-action on $M$. Given $p \in M$, let $S_{p}$ a slice at $p$. We define a tubular neighborhood of the orbit $G(p)$ as the image of $S_{p}$ under the $G$-action,

$$
\operatorname{Tub}(G(p)):=\mu\left(G, S_{p}\right) .
$$

There is a more convenient and specific definition of a tubular neighborhood for a SRF, which will be fundamental in the construction of of composed foliations (Section 2.2).

Given a point $p$ in a singular Riemannian foliation $(M, \mathcal{F})$, an open, relatively compact (an open subset with compact closure) neighborhood $P$ of $p \in M$ in the leaf $L_{p}$ is called a plaque. 
Definition 1.19. For such a plaque, there exists some $\varepsilon>0$ such that the normal exponential map

$$
\begin{aligned}
\exp ^{\perp}: \nu(P) & \longrightarrow M \\
\nu^{\varepsilon}(P) & \longmapsto \exp ^{\perp}\left(\nu^{\varepsilon}(P)\right)=: T u b_{\varepsilon}(P)
\end{aligned}
$$

is a diffeomorphism on the normal bundle of $P$ of radius $\varepsilon, \nu^{\varepsilon}(P)=\{v \in \nu(P) \mid\|v\| \leqslant \varepsilon\}$. The image of $\nu^{\varepsilon}(P)$ under $\exp ^{\perp}$ will be called a distinguished tubular neighborhood around $P$, and denoted $T u b_{\varepsilon}(P)$.

The restriction $\left.\mathcal{F}\right|_{T u b_{\varepsilon}(P)}$ is the foliation in $T u b_{\varepsilon}(P)$ given by the connected components of the intersection $L \cap T u b_{\varepsilon}(P)$, for all $L \in \mathcal{F}$. It may happen that some leaf intersects $T u b_{\varepsilon}(P)$ more than once, in which case that intersections are counted separately.

A distinguished tubular neighborhood comes equipped with a map $\operatorname{Pr}$ called the metric projection, $\operatorname{Pr}: T u b_{\varepsilon}(P) \rightarrow P$, defined by the composition

$$
T u b_{\varepsilon}(P) \stackrel{\exp ^{-1}}{\longrightarrow} \nu^{\varepsilon}(P) \rightarrow P .
$$

If $\varepsilon$ is chosen small enough, this projection sends a point $p^{\prime}$ to the point in $P$ which is closest to $p^{\prime}$, given via horizontal geodesics. For $q \in P$, we denote the fiber $S_{q}:=\operatorname{Pr}^{-1}(q)$ and call it the slice through $q$. In addition, if $y \in T u b_{\varepsilon}(P)$, the connected component $P_{y}$ of $L_{y} \cap T u b_{\varepsilon}(P)$ containing $y$ is called the plaque of $\mathcal{F}$ through $y$ in the neighborhood.

Lemma 1.20 (Homothetic Transformation Lemma). Let $p \in T u b_{\varepsilon}(P)$ a distinguished tubular neighborhood in $(M, \mathcal{F})$ such that $P$ is a plaque around $p$. For each $\lambda \in(0,1)$, the map

$$
\begin{aligned}
h_{\lambda}: T u b_{\varepsilon}(P) & \longrightarrow T u b_{\lambda \varepsilon}(P) \\
\exp ^{\perp}(v) & \longmapsto \exp ^{\perp}(\lambda v),
\end{aligned}
$$

for $v \in T u b_{\varepsilon}(P)$ is a foliated diffeomorphism sending (connected components of) leaves of $\left.\mathcal{F}\right|_{T u b_{\varepsilon}(P)}$ to (connected components of) leaves $\left.\mathcal{F}\right|_{T u b_{\lambda \varepsilon}(P)}$.

The next result is a consequence of the lemma above and it describes the local structure of a stratification. We draw your attention to the second property below, which has an interesting improvement due to Lytchak and Thorbergsson [LT10, Section 4] of Molino's classical results ([MC88, Proposition 6.3]); namely, the geodesic just leaves the stratum a discrete set of times.

Lemma 1.21. Every connected component of a stratum is a (possibly non-complete) manifold. Moreover, every geodesic starting tangent to a stratum and perpendicular to the leaf stays in the stratum for all but a discrete set of times.

The definition and theorem below describe how a singular Riemannian foliation looks locally. Let $(M, \mathcal{F})$ be a SRF and let $S_{p}$ be a slice at a point $p \in M$.

Definition 1.22. The infinitesimal foliation of $\mathcal{F}$ at $p$, denoted by $\left(\nu_{p} L_{p}, \mathcal{F}_{p}\right)$, is defined 
as the partition of $\nu_{p} L_{p}$ whose leaf at $v \in \nu_{p} L_{p}$ is given by

$$
L_{v}:=\left\{w \in \nu_{p} L_{p} \mid \exp _{p} t w \in L_{\exp _{p} t v}\right\}, \quad \text { for } t \text { small enough, }
$$

where $L_{\exp _{p} t v}$ denotes the leaf of $\left(S_{p},\left.\mathcal{F}\right|_{S_{p}}\right)$ through $\exp _{p} t v$.

Notice that the Homothetic Transformation Lemma guarantees that $L_{v}$ is well defined: if for some small enough $t_{0}$, $\exp _{p} t_{0} w$ belongs to the same leaf of $\exp _{p} t_{0} v$, then $\exp _{p} t w$ lies on the same leaf of $\exp _{p} t v$, for $t \in\left(0, t_{0}\right)$. For these local foliations, there are some properties we state below.

Theorem 1.23. For a infinitesimal foliation $\left(\nu_{p} L_{p}, \mathcal{F}_{p}\right)$ of $(M, \mathcal{F})$ it holds the following:

(a) The normal exponential map $\exp _{p}: \nu_{p}^{\varepsilon} L_{p} \rightarrow M$ sends the leaves of $\mathcal{F}_{p}$ to the leaves of $\left(S_{p},\left.\mathcal{F}\right|_{S_{p}}\right)$.

(b) The foliation $\left(\nu_{p} L_{p}, \mathcal{F}_{p}\right)$ is invariant under homotheties, i.e., if $v \in \nu_{p} L_{p}$, then $L_{\lambda v}=$ $\lambda \cdot L_{v}$, for a non-zero real number $\lambda$.

(c) $\left(\nu_{p} L_{p}, \mathcal{F}_{p}\right)$ is a SRF with respect to the flat metric at $p$.

\subsection{Clifford systems, their algebras and representations}

The construction of an infinite collection of isoparametric hypersurfaces with four principal curvatures given by Ferus, Karcher and Münzner in [FKM81] is based on representations of Clifford algebras. With the intention of reproducing their work, we need first to review some facts about Clifford algebras, their representations and systems.

\section{Clifford algebras}

For each integer $m \geqslant 0$, the Clifford algebra $C_{m}$ is the associative algebra over $\mathbb{R}$ that is generated by a unit element 1 and the elements $e_{1}, \ldots, e_{m}$ subject only to the relations

$$
e_{i}^{2}=-1, \quad e_{i} e_{j}=-e_{j} e_{i}, \quad i \neq j, \quad 1 \leqslant i, j \leqslant m .
$$

In other words, $C_{m}$ is an additive abelian group which has the structure of both a ring and a vector space over $\mathbb{R}$.

An equivalent definition is that the Clifford algebra is constructed from a real vector space $V=\operatorname{span}\left\{e_{1}, \ldots, e_{m}\right\}$ with a positive definite inner product $\langle$,$\rangle , by taking the quotient$ of the tensor algebra $T(V)$ by the ideal $x \otimes y+y \otimes x-2\langle x, y\rangle 1$. We have that $V$ embeds naturally in $C_{m}$ and

$$
\forall x, y \in V, \quad x \cdot y+y \cdot x=-2\langle x, y\rangle \cdot 1 .
$$

We can see for instance that if $m=0, C_{0}$ is isomorphic to $\mathbb{R}, C_{1}$ is isomorphic to the complex numbers $\mathbb{C}$ with $e_{1}$ equals to the complex number $i$, and $C_{2}$ is isomorphic to the 
quaternions $\mathbb{H}$ with the correspondence

$$
e_{1}=i, \quad e_{2}=j, \quad e_{i} e_{j}=i j=k .
$$

Note that the set

$$
\left\{1, e_{i_{1}} \cdots e_{i_{k}} \mid i_{1}<\cdots<i_{k}, 1 \leqslant k \leqslant m\right\}
$$

forms a basis for $C_{m}$ over $\mathbb{R}$ and hence $C_{m}$ has dimension $2^{m}$ as a real vector space.

\section{Clifford representations}

Atiyah, Bott and Shapiro in [ABS64] determined the classification of all Clifford algebras by means of their irreducible representations, which will be fundamental in the study of the FKM hypersurfaces.

Let $e_{1}, \ldots, e_{m}$ be a basis for $V$ and $E_{1}, \ldots, E_{m}$ be skew-symmetric $l \times l$ matrices with real entries satisfying

$$
E_{i}^{2}=-I, \quad E_{i} E_{j}=-E_{j} E_{i}, \quad i \neq j, \quad 1 \leqslant i, j \leqslant m,
$$

where $I$ is the $l \times l$ identity matrix. Notice from the definition that every $E_{i}$ is also an orthogonal matrix since, for any vectors $u, v$ in $\mathbb{R}^{n}$,

$$
\left\langle E_{i} u, E_{i} v\right\rangle=\left\langle u, E_{i}^{T} E_{i} v\right\rangle=\left\langle u,-E_{i} E_{i} v\right\rangle=\langle u, I v\rangle=\langle u, v\rangle .
$$

A representation $\rho$ of a Clifford algebra $C_{m}$ on $\operatorname{End}\left(\mathbb{R}^{l}\right)$ of degree $l$

$$
\rho: C_{m} \longrightarrow \operatorname{End}\left(\mathbb{R}^{l}\right)
$$

is an algebra homomorphism such that $\rho(1)=I$ and $\rho\left(e_{i}\right)=E_{i}$, for $1 \leqslant i \leqslant m$.

Example 1.24. We already know that the Clifford algebra $C_{1}$ is $\mathbb{C}=\operatorname{span}\left\{1, e_{1}=i\right\}$. Let $l=2$, a Clifford representation of $C_{1}$ on $\operatorname{End}\left(\mathbb{R}^{2}\right)$ could be

$$
\begin{aligned}
\rho: \mathbb{C} & \longrightarrow M_{2}(\mathbb{R}) \\
1 & \longmapsto\left[\begin{array}{ll}
1 & 0 \\
0 & 1
\end{array}\right]=I \\
e_{1}=i & \longmapsto\left[\begin{array}{cc}
0 & -1 \\
1 & 0
\end{array}\right]=E_{1} .
\end{aligned}
$$

Note that any other complex number $a+b i$ is written in this case as $\left[\begin{array}{cc}a & -b \\ b & a\end{array}\right]$.

A representation $\rho: C_{m-1} \rightarrow \operatorname{End}\left(\mathbb{R}^{l}\right)$, for $m \geqslant 1$, is called irreducible if it has no proper invariant subspaces. Otherwise, $\rho$ is reducible of degree $l$, for $l=k \delta(m)$ and $k>1$ an integer, is formed by taking a direct sum of $k$ irreducible representations of $C_{m-1}$ of degree $\delta(m)$, where the function $\delta(m)$ is given in Table 1.1. 
The complete classification of Clifford algebras was determined by Atiyah, Bott and Shapiro and is found in the second row of the table, where the following terminology is used: Let $A(k)$ denote the algebra of $k \times k$ matrices with entries from the algebra $A$. The multiplication in $A(k)$ is matrix multiplication defined using the operations of addition and multiplication in the algebra $A$. The direct sum $A_{1} \oplus A_{2}$ is the Cartesian product $A_{1} \times A_{2}$ with all algebra operations defined coordinatewise. Those three authors also showed that each Clifford algebra $C_{m-1}$ has an irreducible representation of degree $l$ if and only if $l=\delta(m)$, as can be seen in Table 1.1 as well.

\begin{tabular}{|c||c|c|c|c|c|c|c|c|c|}
\hline$m$ & 1 & 2 & 3 & 4 & 5 & 6 & 7 & 8 & $8+n$ \\
\hline$C_{m-1}$ & $\mathbb{R}$ & $\mathbb{C}$ & $\mathbb{H}$ & $\mathbb{H} \oplus \mathbb{H}$ & $\mathbb{H}(2)$ & $\mathbb{C}(4)$ & $\mathbb{R}(8)$ & $\mathbb{R}(8) \oplus \mathbb{R}(8)$ & $C_{n-1}(16)$ \\
\hline$\delta(m)$ & 1 & 2 & 4 & 4 & 8 & 8 & 8 & 8 & $16 \delta(n)$ \\
\hline
\end{tabular}

Table 1.1: Clifford algebras and degree of their irreducible representations.

\section{Clifford systems}

In this section we finally present the most relevant algebraic object we will use to construct the Clifford foliations and the new examples of singular Riemannian foliations found by Radeschi.

Let $\operatorname{Sym}_{n}(\mathbb{R})$ the space of $n \times n$ symmetric matrices with entries on $\mathbb{R}$ with the standard inner product $\langle A, B\rangle:=\frac{1}{n} \operatorname{trace}(A B)$. The $(m+1)$-tuple $\left(P_{0}, \ldots, P_{m}\right)$ with $P_{i} \in \operatorname{Sym}_{n}(\mathbb{R})$ is called a (symmetric) Clifford system $C$ of rank $m+1$ on $\mathbb{R}^{n}$ if the $P_{i}$ satisfy

$$
P_{i}^{2}=I, \quad P_{i} P_{j}=-P_{j} P_{i}, \quad i \neq j, \quad 0 \leqslant i, j \leqslant m
$$

Similarly as in eq. (1.4) for the Clifford algebra, observe that the $P_{i}$ are orthogonal as well.

Let $C_{m-1}$ a Clifford algebra constructed from a vector space $V$. Following the description of Radeschi, the restriction to $V$ of a representation $\rho: C_{m-1} \rightarrow \operatorname{End}\left(\mathbb{R}^{n}\right)$ of $C_{m-1}$,

$$
C:=\left.\rho\right|_{V}: V \rightarrow \operatorname{End}\left(\mathbb{R}^{n}\right)
$$

is a Clifford system on $\mathbb{R}^{n}$ such that, for an orthonormal basis $\left\{e_{1}, \ldots, e_{m-1}\right\}$ of $V$, their images under $\rho$ are the $P_{i}=\rho\left(e_{i}\right)$. From now on, we will write $\mathbb{R}_{C}$ as being the image $\rho(V)$. We will postpone giving an example of a Clifford system to Section 1.2, where will take in advantage the correspondence between Clifford representations and Clifford systems constructed in the next subchapter.

It follows from eq. (1.2) that a Clifford system inherits the following property for any two elements $P, Q$ belonging to it:

$$
P Q+Q P=2\langle P, Q\rangle I
$$

That characteristic will be used in several computations throughout this text. In fact, its 
first application is related to bases and eigenspaces on a Clifford system, as we show below.

Let $\mathbb{S}_{C}$ denote the unit sphere in $\mathbb{R}_{C}$. For any $P \in \mathbb{S}_{C}, P^{2}=I$ and therefore the matrix $P$ has eigenvalues \pm 1 , with corresponding eigenspaces $E_{ \pm}(P)$. Every $Q$ in $\mathbb{R}_{C}$ can be written in the basis $\left(P_{0}, \ldots, P_{m}\right)$ as

$$
Q=\sum_{i=1}^{m}\left\langle Q, P_{i}\right\rangle P_{i}
$$

We obtain, applying eq. (1.6) to orthogonal elements $P, Q$ :

$$
P Q=-Q P+2\langle P, Q\rangle I=-Q P
$$

An immediate conclusion is that $Q$ takes the positive eigenspace $E_{+}(P)$ isomorphically onto the negative eigenspace $E_{-}(P)$ and vice versa. In particular, $\operatorname{dim} E_{+}(P)=\operatorname{dim} E_{-}(P)$, and since $\mathbb{R}^{n}$ splits as the sum $E_{+}(P) \oplus E_{-}(P), n$ is always even dimensional, and henceforth in this text we will write $n=2 l$.

It is significant to inquire if there are some fundamental "cells" which could form any other Clifford systems from simpler ones by means of a specific operation. In the light of this question we give the following definitions. Given two Clifford systems $\left(P_{0}, \ldots, P_{m}\right)$ on $C: V \rightarrow \operatorname{End}\left(\mathbb{R}^{2 l}\right)$ and $\left(Q_{0}, \ldots, Q_{m}\right)$ on $C^{\prime}: V \rightarrow \operatorname{End}\left(\mathbb{R}^{2 r}\right)$, we can produce a new Clifford system, called the direct sum, $\left(P_{0} \oplus Q_{0}, \ldots, P_{m} \oplus Q_{m}\right)$ on $C \oplus C^{\prime}: V \rightarrow \operatorname{End}\left(\mathbb{R}^{2 l} \oplus \mathbb{R}^{2 r}\right)=$ $\operatorname{End}\left(\mathbb{R}^{2(l+r)}\right)$ by defining $\left(C \oplus C^{\prime}\right)(x, y)=\left(C(x), C^{\prime}(y)\right)$, that is, $\left(P_{i} \oplus Q_{i}\right)(x, y)=\left(P_{i} x, Q_{i} y\right)$. The system $C \oplus C^{\prime}$ is called a reducible system. Any Clifford system that cannot be written as a non-trivial sum is called irreducible. Furthermore, if $C$ is an irreducible Clifford system of rank $m+1$ on $\mathbb{R}^{2 l}$ then $l=\delta(m)$, where the function $\delta(m)$ is given in Table 1.1.

Two Clifford systems $\left(P_{0}, \ldots, P_{m}\right),\left(Q_{0}, \ldots, Q_{m}\right)$ on $C, C^{\prime}: V \rightarrow \operatorname{Sym}_{2 l}(\mathbb{R})$, respectively, are algebraically equivalent if there is an isometry $A \in \mathrm{O}\left(\mathbb{R}^{2 l}\right)$ such that $C^{\prime}=A^{-1} \circ C \circ A$, in other terms if $Q_{i}=A P_{i} A^{T}$, for $0 \leqslant i \leqslant m$. And they are called geometrically equivalent if there is an isometry $A \in \mathrm{O}\left(\mathbb{R}^{2 l}\right)$ such that $\mathbb{R}_{C^{\prime}}=\mathbb{R}_{A^{-1} \circ C \circ A}$, or equivalently, if there exists $B \in \mathrm{O}\left(\operatorname{span}\left\{P_{0}, \ldots, P_{m}\right\} \subseteq \operatorname{Sym}_{2 l}(\mathbb{R})\right)$ such that $\left(Q_{0}, \ldots, Q_{m}\right)$ and $\left(B P_{0}, \ldots, B P_{m}\right)$ are algebraically equivalent, that is, $Q_{i}=A B P_{i} A^{T}, 0 \leqslant i \leqslant m$, for $A \in \mathrm{O}(2 l)$.

\section{Correspondence between Clifford systems and their representations}

There is an explicit correspondence between Clifford systems on $\mathbb{R}^{2 l}$ and representations of Clifford algebras on $\mathbb{R}^{l}$ which is used in the construction of the FKM examples to deduce several properties about the former ones from known facts about the latter ones.

Suppose that $E_{1}, \ldots, E_{m-1}$ form a representation of a Clifford algebra $C_{m-1}$ on $\mathbb{R}^{l}$ as in eq. (1.3). Now we write $\mathbb{R}^{2 l}=\mathbb{R}^{l} \oplus \mathbb{R}^{l}$ and define transformations $P_{0}, \ldots, P_{m}$ by

$$
P_{0}(u, v)=(u,-v), \quad P_{1}(u, v)=(v, u), \quad P_{1+i}(u, v)=\left(E_{i} v,-E_{i} u\right), \quad 1 \leqslant i \leqslant m-1 .
$$


It follows that $\left(P_{0}, \ldots, P_{m}\right)$ are symmetric and hold the conditions in eq. (1.5) and so they produce a Clifford system of rank $m+1$ on $\mathbb{R}^{2 l}$.

Conversely, suppose $\left(P_{0}, \ldots, P_{m}\right)$ is a Clifford system of rank $m+1$ on $\mathbb{R}^{2 l}$ (eq. (1.5)). Setting $P:=P_{i}$ and $Q:=P_{j}$ in eq. (1.6) we know that every $P_{j}$ interchanges the eigenspaces $E_{ \pm}\left(P_{i}\right)$ for $i \neq j$. This shows not only that both spaces have the same dimension but also that $P_{i}$ has trace zero. Now we can for instance identify $\mathbb{R}^{l}$ with $E_{+}\left(P_{0}\right)$ and define the transformation $E_{i}: \mathbb{R}^{l} \rightarrow \mathbb{R}^{l}, 1 \leqslant i \leqslant m-1$, to be the restriction to $E_{+}\left(P_{0}\right)$ of the transformation $P_{1} P_{1+i}$, that is,

$$
E_{i}:=\left.P_{1} P_{1+i}\right|_{E_{+}\left(P_{0}\right)}
$$

Then a computation gives that $E_{1}, \ldots, E_{m-1}$ are skew-symmetric and determine a representation $C_{m-1}$ of a Clifford algebra on $\mathbb{R}^{l}$ (see [Cec13, p. 99-101] for the details of this correspondence).

Now it is appropriate to present an example of a Clifford system we owe from the last section.

Example 1.25. Recall that in Example 1.24 we found the Clifford algebra representation of $\mathbb{C}$ on $\operatorname{End}\left(\mathbb{R}^{2}\right)$ when $m=l=2$. The matrices

$$
P_{0}=\left[\begin{array}{cc}
I & 0 \\
0 & -I
\end{array}\right]_{4}, \quad P_{1}=\left[\begin{array}{ll}
0 & I \\
I & 0
\end{array}\right]_{4}, \quad P_{2}=\left[\begin{array}{cc}
0 & E_{1} \\
-E_{1} & 0
\end{array}\right]_{4},
$$

with $I$ the $2 \times 2$ identity matrix, form its corresponding Clifford system of rank $m+1=3$ on $\mathbb{R}^{2 l}=\mathbb{R}^{4}=\mathbb{R}^{2} \oplus \mathbb{R}^{2}$ where we have employed the construction just exhibited.

Owing to that connection, Ferus, Karcher and Münzner deduced that a Clifford system is irreducible if and only if its corresponding Clifford algebra representation is irreducible. For instance there exists an irreducible Clifford system $\left(P_{0}, \ldots, P_{m}\right)$ on $\mathbb{R}^{2 l}$ if and only if $l=\delta(m)$ as in Table 1.1. They went further in this characterization and showed that, for $m \equiv 0(\bmod 4)$, there are just two algebraic equivalence classes of Clifford systems, distinguished by the basis $\left(P_{0}, P_{1}, \ldots, P_{m}\right)$ or $\left(-P_{0}, P_{1}, \ldots, P_{m}\right)$. This also means that there is one geometric class of irreducible Clifford systems for that $m$. On the other hand, when $m \not \equiv 0(\bmod 4)$, there exists exactly one algebraic (thus only one geometric) equivalence class of Clifford systems $\left(P_{0}, \ldots, P_{m}\right)$ on $\mathbb{R}^{2 l}$ with $l=k \delta(m)$ and $k$ a positive integer.

It is quite convenient having a subset of a Clifford system that not only generates all the given space but also one whose elements are unit in some sense, similar to an orthonormal basis in a vector space. In a Clifford system $C$ of rank $m+1$ on $\mathbb{R}^{2 l}$, that role is played by the unit sphere $\mathbb{S}_{C}$ in $\mathbb{R}_{C}$, which from now it will be called the Clifford sphere determined by $C$. Together with the stated in page 18 , the next theorem gives useful properties of Clifford spheres.

Theorem 1.26 (Clifford sphere properties). The Clifford sphere $\mathbb{S}_{C}$ in $\mathbb{R}_{C}$ has the following properties: 
(a) For each $P \in \mathbb{S}_{C}, P^{2}=I$. Conversely, if $\mathbb{S}_{C}$ is the unit sphere in a linear subspace $W$ spanned by $\mathbb{S}_{C}$ in $\operatorname{Sym}_{2 l}(\mathbb{R})$ such that $P^{2}=I$ for all $P \in \mathbb{S}_{C}$, then every orthonormal basis of $W$ is a Clifford system on $\mathbb{R}^{2 l}$.

(b) Two Clifford systems are geometrically equivalent if and only if their Clifford spheres are conjugate to one another under an orthogonal transformation on $\mathbb{R}^{2 l}$.

(c) The function

$$
H(x)=\sum_{i=0}^{m}\left\langle P_{i} x, x\right\rangle^{2}
$$

depends only on $\mathbb{S}_{C}$ and not on the choice of orthonormal basis $\left(P_{0}, \ldots, P_{m}\right)$. For $P \in \mathbb{S}_{C}$ we have

$$
H(P x)=H(x)
$$

for all $x \in \mathbb{R}^{2 l}$.

(d) For an orthonormal set $\left\{Q_{1}, \ldots, Q_{r}\right\}$ in $\mathbb{S}_{C}$ since $Q_{i} Q_{j}=-Q_{j} Q_{i}$, for $i \neq j$, we have

$$
\begin{aligned}
& Q_{1} \cdots Q_{r} \quad \text { is symmetric if } r \equiv 0,1 \quad(\bmod 4), \\
& Q_{1} \cdots Q_{r} \quad \text { is skew-symmetric if } r \equiv 2,3 \quad(\bmod 4) .
\end{aligned}
$$

Furthermore, the product $Q_{1} \cdots Q_{r}$ is uniquely determined by a choice of orientation of $\operatorname{span}\left\{Q_{1}, \cdots, Q_{r}\right\}$.

(e) For $P, Q \in \mathbb{S}_{C}$ and $x \in \mathbb{R}^{2 l}$, we have

$$
\langle P x, Q x\rangle=\langle P, Q\rangle\langle x, x\rangle .
$$

We reference the reader to see the proof of this theorem in [Cec13, p. 102-105].

\subsection{FKM isoparametric hypersurfaces}

We now discuss the last preliminar notion that we need before focusing in Clifford and composed foliations, the recent classes introduced by Radeschi. Those recent examples of SRF of higher codimension are a generalization of the construction of Ferus, Karcher and Münzner [FKM81], now called FKM examples. Roughly speaking, an FKM family is a SRF of isoparametric hypersurfaces with $g=4$ distinct principal curvatures in a sphere, whose leaves of maximal dimension have codimension 1. We study in this section such FKM examples, which are constructed from Clifford systems.

Throughout this section we will work with $C$ a Clifford system of rank $m+1$ on $\mathbb{R}^{2 l}$, $l=k \delta(m)$, and we will fix a basis $P_{0}, \ldots, P_{m}$ for it.

\section{Isoparametric functions}

Recall that a function $F: \mathbb{R}^{n+2} \rightarrow \mathbb{R}$ homogeneous of degree $g$ if $f(t x)=t^{g} f(x)$, for any $t$ in $\mathbb{R}$ and $g$ a positive integer. We draw your attention to the polynomial of the next 
example since it will be the starting point for developing FKM isoparametric hypersurfaces on the sphere $\mathbb{S}^{2 l-1}$.

Example 1.27. Consider the polynomial $F: \mathbb{R}^{2 l} \rightarrow \mathbb{R}$ defined as

$$
F(x)=\langle x, x\rangle^{2}-2 \sum_{i=0}^{m}\left\langle P_{i} x, x\right\rangle^{2} .
$$

It is easy checking $F$ is a homogeneous function of degree $g=4$ since

$$
F(t x)=\langle t x, t x\rangle^{2}-2 \sum_{i=0}^{m}\left\langle P_{i}(t x), t x\right\rangle^{2}=t^{4}\langle x, x\rangle^{2}-2 t^{4} \sum_{i=0}^{m}\left\langle P_{i} x, x\right\rangle^{2}=t^{4} F(x) .
$$

By Euler's theorem, it is known that for a homogeneous function of degree $g$

$$
\left\langle\operatorname{grad}^{E} F, x\right\rangle=g F(x),
$$

for any $x \in \mathbb{R}^{n+2}$ and where we have used the superscript $E$ to denote the Euclidean gradient of $F$. Since we will work with the restriction of $F$ to the sphere $S^{n+1}$, we similarly denote the gradient of it by $\operatorname{grad}^{S} F$, and the respective Laplacians by $\Delta^{E} F$ and $\Delta^{S} F$. Now we proceed defining an isoparametric function for a real space form.

Definition 1.28. Let $M^{n+1}$ a real space form. A nonconstant smooth function $F: M^{n+1} \rightarrow$ $\mathbb{R}$ is said to be isoparametric if their differential parameters $\left|\operatorname{grad}^{E} F\right|^{2}$ and $\Delta F$, the Laplacian of $F$, are constant on each level set of $F$. More precisely, if there exist smooth functions $\phi_{1}$ and $\phi_{2}$ from $\mathbb{R}$ to $\mathbb{R}$ such that

$$
\left|\operatorname{grad}^{E} F\right|^{2}=\phi_{1}(F), \quad \Delta F=\phi_{2}(F) .
$$

The following theorem relates these differential operators with a homogeneous function of degree $g$ (see [CR15, p. 112-113] for a detailed proof).

Theorem 1.29. Let $F: \mathbb{R}^{n+2} \rightarrow \mathbb{R}$ be a homogeneous function of degree $g$. Then

(a) $\left|\operatorname{grad}^{S} F\right|^{2}=\left|\operatorname{grad}^{E} F\right|^{2}-g^{2} F^{2}$,

(b) $\Delta^{S} F=\Delta^{E} F-g(g-1) F-g(n+1) F$.

Example 1.30. We are going to show that $F_{0}:=\left.F\right|_{\mathbb{S}^{2 l-1}}$, the restriction of the function $F$ (defined in eq. (1.7)) to the sphere $\mathbb{S}^{2 l-1}$,

$$
F_{0}(x)=1-2 \sum_{i=0}^{m}\left\langle P_{i} x, x\right\rangle^{2}
$$

is an isoparametric function, using the homogeneity of $F$. The Euclidean gradient of $F$ is

$$
\begin{aligned}
\operatorname{grad}^{E} F & =2\langle x, x\rangle 2 x+2 \sum_{i=0}^{m} 2\left\langle P_{i} x, x\right\rangle 2 P_{i} x \\
& =4\langle x, x\rangle x+8 \sum_{i=0}^{m}\left\langle P_{i} x, x\right\rangle P_{i} x
\end{aligned}
$$


thus,

$$
\begin{aligned}
\left|\operatorname{grad}^{E} F\right|^{2} & =\left\langle 4\langle x, x\rangle x+8 \sum_{i=0}^{m}\left\langle P_{i} x, x\right\rangle P_{i} x, 4\langle x, x\rangle x+8 \sum_{i=0}^{m}\left\langle P_{i} x, x\right\rangle P_{i} x\right\rangle \\
& =16\langle x, x\rangle^{3}-64\langle x, x\rangle \sum_{i=0}^{m}\left\langle P_{i} x, x\right\rangle^{2}+64 \sum_{i, j=0}^{m}\left\langle P_{i} x, x\right\rangle\left\langle P_{j} x, x\right\rangle\left\langle P_{i} x, P_{j} x\right\rangle \\
& =16\langle x, x\rangle^{3}-64\langle x, x\rangle \sum_{i=0}^{m}\left\langle P_{i} x, x\right\rangle^{2}+64 \sum_{i, j=0}^{m}\left\langle P_{i} x, x\right\rangle\left\langle P_{j} x, x\right\rangle\left\langle P_{i}, P_{j}\right\rangle\langle x, x\rangle \\
& =16\langle x, x\rangle^{3}-64\langle x, x\rangle \sum_{i=0}^{m}\left\langle P_{i} x, x\right\rangle^{2}+64\langle x, x\rangle \sum_{i, j=0}^{m}\left\langle P_{i} x, x\right\rangle\left\langle P_{j} x, x\right\rangle \delta_{i j} \\
& =16\langle x, x\rangle^{3}-64\langle x, x\rangle \sum_{i=0}^{m}\left\langle P_{i} x, x\right\rangle^{2}+64\langle x, x\rangle \sum_{i=0}^{m}\left\langle P_{i} x, x\right\rangle^{2} \\
& =16\langle x, x\rangle^{3} \\
& =16\|x\|^{6},
\end{aligned}
$$

where in the third line we used Theorem $1.26(\mathrm{e})$ and in the fourth one that $\left(P_{0}, \ldots, P_{m}\right)$ is a basis for $\mathbb{R}^{2 l}$. To compute the Euclidean laplacian of $F$ we are going to use the next identity which holds for any smooth function $h: \mathbb{R}^{2 l} \rightarrow \mathbb{R}$ :

$$
\begin{aligned}
\Delta^{E} h^{2} & =\sum_{i}^{2 l} \frac{\partial}{\partial x_{i}}\left(h^{2}\right) \\
& =\sum_{i}^{2 l} \frac{\partial\left(2 h \operatorname{grad}^{E} h\right)}{\partial x_{i}} \\
& =2 \sum_{i}^{2 l} \frac{\partial h}{\partial x_{i}} \operatorname{grad}^{E} h+2 h \sum_{i}^{2 l} \frac{\partial\left(\operatorname{grad}^{E} h\right)}{\partial x_{i}} \\
& =2\left|\operatorname{grad}^{E} h\right|^{2}+2 h \Delta^{E} h .
\end{aligned}
$$

Taking the Euclidean laplacian for $F$, and applying eq. (1.10) in $\Delta\langle x, x\rangle^{2}$ and $\Delta\left\langle P_{i} x, x\right\rangle^{2}$, we get

$$
\begin{aligned}
\Delta^{E} F= & \Delta^{E}\langle x, x\rangle^{2}-2 \sum_{i=0}^{m} \Delta^{E}\left\langle P_{i} x, x\right\rangle^{2} \\
= & 2\left|\operatorname{grad}^{E}\langle x, x\rangle\right|^{2}+2\langle x, x\rangle \Delta^{E}\langle x, x\rangle \\
& -2 \sum_{i=0}^{m}\left(2\left|\operatorname{grad}\left\langle P_{i} x, x\right\rangle\right|^{2}+2\left\langle P_{i} x, x\right\rangle \Delta^{E}\left\langle P_{i} x, x\right\rangle\right) \\
= & 8\langle x, x\rangle+2\langle x, x\rangle 2(2 l)-2 \sum_{i=0}^{m}\left(8\left\langle P_{i} x, P_{i} x\right\rangle+2\left\langle P_{i} x, x\right\rangle 2 \operatorname{trace}\left(P_{i}\right)\right) \\
= & 8\langle x, x\rangle+8 l\langle x, x\rangle-16 \sum_{i=0}^{m}\left\langle P_{i}, P_{i}\right\rangle\langle x, x\rangle
\end{aligned}
$$




$$
\begin{aligned}
& =8\langle x, x\rangle+8 l\langle x, x\rangle-16(m+1)\langle x, x\rangle \\
& =8(l-2 m-1)\langle x, x\rangle \\
& =8(l-2 m-1)\|x\|^{2}
\end{aligned}
$$

where we used in the fourth line that $\operatorname{trace}\left(P_{i}\right)=0$, for all $i$, and Theorem $1.26(\mathrm{e})$. Applying Theorem 1.29, together with eqs. (1.9) and (1.11), and having $\|x\|^{2}=1$ in the unit sphere $\mathbb{S}^{2 l-1}$, we obtain

$$
\left|\operatorname{grad}^{S} F\right|^{2}=16-16 F^{2}=16\left(1-F^{2}\right)
$$

and

$$
\Delta^{S} F=8(l-2 m-1)-12 F-4(2 l-1) F=8(l-2 m-1)-8(l+1) F .
$$

Therefore, $\left|\operatorname{grad}^{S} F\right|^{2}$ and $\Delta^{E} F$ are both functions of $F$ itself and thus its restriction $F_{0}$ to $\mathbb{S}^{2 l-1}$ is an isoparametric function.

Remark 1.31. Taking the restriction $F_{0}$ of $F$ to $\mathbb{S}^{2 l-1}$ in eq. (1.12) we have that

$$
\left|\operatorname{grad}^{S} F_{0}\right|^{2}=16\left(1-F_{0}^{2}\right) \text {. }
$$

Since the left hand side of this equation is nonnegative, then $0 \leqslant 16\left(1-F_{0}^{2}\right)$, or equivalently $F_{0} \leqslant \pm 1$. Thus, the image of $F_{0}$ is contained in the closed interval $[-1,1]$. Moreover, $F_{0}$ is a non constant, continuous function on the compact set $\mathbb{S}^{2 l-1}$, then it attains its (different) minimum and maximum values, which must be 1 and -1 , respectively, since $\operatorname{grad}^{S} F_{0}$ is non zero for every point where $F_{0}$ is different from \pm 1 . From this we conclude that the closed interval $[-1,1]$ is the image of $F_{0}=\left.F\right|_{\mathbb{S}^{2 l-1}}$.

Notice that any level set $F_{0}^{-1}(t)$, for $t \in(-1,1)$, is a compact hypersurface since it is a closed subset in a compact space and $\operatorname{grad}^{S} F_{0}$ is never zero on these level sets. On the other hand, when $t= \pm 1$, the gradient $\operatorname{grad}^{S} F_{0}$ is zero and its corresponding level sets $M_{+}:=F_{0}^{-1}(1)$ and $M_{-}:=F_{0}^{-1}(-1)$ are submanifolds of codimension greater than one in $\mathbb{S}^{2 l-1}$. These kind of submanifolds are called focal and they play a crucial role in the theory of isoparametric hypersurfaces as well as in singular Riemannian foliations. We will give a precise definition of them later on this chapter.

Münzner proved that each level set of $F_{0}$ is connected, see for example [CR15, Section 3.6].

\section{Isoparametric hypersurfaces}

It turns out that associated to an isoparametric function on a real space form, there exists a family of isoparametric hypersurfaces in terms of its level sets. We start this section giving a geometric definition of that latter objects in terms of their principal curvatures, and then we will relate them from an analytic point of view to their corresponding isoparametric functions.

Definition 1.32. A connected hypersurface $M$ immersed in a real space form is said to be isoparametric if it has constant principal curvatures. 
In the theory of isoparametric hypersurfaces developed essentially by Cartan and Münzner, we find out for an isoparametric function, each level set has constant principal curvatures, and consequently, from the definition above, it is also an isoparametric hypersurface. We state this below (for a reference see, for example, chapter 3 in [CR15]).

Proposition 1.33. If $F: M^{n+1} \rightarrow \mathbb{R}$ is an isoparametric function on a real space form, then each level hypersurface of $F$ has constant principal curvatures and then it is an isoparametric hypersurface.

Example 1.34. Continuing with Example 1.27, the level sets of $F_{0}: \mathbb{S}^{2 l-1} \rightarrow[-1,1]$ are of the form

$$
M_{t}=\left\{z \in \mathbb{S}^{2 l-1} \mid F_{0}(z)=\cos (4 t)\right\}, \quad 0 \leqslant t \leqslant \frac{\pi}{4},
$$

and they constitute an isoparametric family of hypersurfaces in $\mathbb{S}^{2 l-1}$. The values of $t=0, \frac{\pi}{4}$ represent the focal submanifolds corresponding to the extreme values in the interval $[-1,1]$, as we explained in Remark 1.31: for $t=0, M_{0}$ and $F_{0}(z)=1$; and for $t=\frac{\pi}{4}, M_{\frac{\pi}{4}}$ and $F_{\frac{\pi}{4}}(z)=-1$.

In the case of an isoparametric hypersurface $M$ in the sphere $\mathbb{S}^{2 l-1}$, Münzner showed that the corresponding isoparametric function comes from a homogeneous polynomial of degree $g$, where $g$ is also the number of distinct principal curvatures of $M$. For instance, in Example 1.27, the isoparametric hypersurfaces of $F_{0}$ have $g=4$ distinct constant principal curvatures.

\section{Parallel hypersurfaces and tubes in the unit sphere $\mathbb{S}^{n+k}$}

Besides the analytic description of an isoparametric family of hypersurfaces in a real space form we did in the previous section, it is also possible characterize them by a geometric approach. Such a viewpoint is through parallel hypersurfaces and tubes and their focal sets. A family of parallel hypersurfaces to a hypersurface with constant principal curvatures in a real space form is equivalent to an isoparametric family of hypersurfaces in terms of level sets of an isoparametric function. Theorems 1.37 and 1.40 provide the explicit expression for principal curvatures of tubes and parallel hypersurfaces in the unit sphere $\mathbb{S}^{n+k}$, and prove those curvatures are constant as well, so they form an isoparametric family. As we will see at the end of this chapter, an isoparametric FKM foliation can be seen a family of tubes with $g=4$ principal curvatures, hence our interest in the topics of this section.

Even though the theory in this section is restricted to submanifolds in a sphere, it can be generalized to any space form and the proofs are similar. We adapted the proofs for the principal values of tubes and parallel hypersurfaces to the sphere case from [CR15, Section 2.2 and 2.3], and we also recommend that book for a further reference on this matter.

Let $f: M^{n} \rightarrow \mathbb{S}^{n+k}$ be an immersion of codimension $k \geqslant 1$ and define the normal bundle of $f(M)$

$$
N M:=\left\{(p, \xi) \mid p \in M, \xi \in T_{p}^{\perp} M\right\}
$$


where $T_{p}^{\perp} M$ is the normal space to $f(M)$ at the point $f(p) \in M$. Its natural projection is $\pi: N M \rightarrow M$ defined by $\pi(p, \xi)=p$. Let $\eta$ be a local section of $N M$. For any vector $X$ in the tangent space $T_{p} M$, we have the fundamental equation

$$
\tilde{\nabla}_{d f(X)} \eta=-d f\left(A_{\eta} X\right)+\nabla_{d f(X)}^{\perp} \eta
$$

where $\tilde{\nabla}$ is the Levi-Civita connection in $\mathbb{S}^{n+k}, A_{\eta}$ is the shape operator determined by the normal vector $\eta(p)$ and $\nabla^{\perp}$ is the connection in the normal bundle. Remember that the map $(x, \xi) \rightarrow A_{\xi}$ from the normal bundle into the space of symmetric tensors of type $(1,1)$ on $M$ is smooth. We call a principal curvature of $A_{\xi}$ to be an eigenvalue $\lambda$ of $A_{\xi}$. Its corresponding eigenvector is called a principal vector. Since $A_{t \xi}=t A_{\xi}$, for $t \in \mathbb{R}$, it is enough to know the principal curvatures on the unit normal bundle of $\mathrm{M}$, which is denoted by $B M$.

Definition 1.35. Let exp : $T \mathbb{S}^{n+k} \rightarrow \mathbb{S}^{n+k}$ the exponential map of $\mathbb{S}^{n+k}$. The normal exponential map is the restriction of exp to the normal bundle $N M$ of the submanifold $M$,

$$
\begin{aligned}
E:=\left.\exp \right|_{N M}: N M & \longrightarrow \mathbb{S}^{n+k} \\
(x, \xi) & \longmapsto E(x, \xi)=\exp _{x}(\xi)=\gamma_{\xi}(1)
\end{aligned}
$$

This means $E(x, \xi)$ is the point of $\mathbb{S}^{n+k}$ reached by traversing a distance $|\xi|$ along the geodesic in $\mathbb{S}^{n+k}$ with initial point $f(x)$ and initial tangent vector $\xi$.

Since we will looking for the critical values of $E$, that is, the points where its differential $d E$ is singular, we will restrict our attention to points in the normal bundle $N M$ that do not belong to the 0-section, since we can always find a neighborhood in that section where the exponential map (and consequently its restriction $E$ ) is a diffeomorphism.

Definition 1.36. A point $p \in \mathbb{S}^{n+k}$ is called a focal point of $(M, x)$ of multiplicity $m$ if $p=E(x, \xi)$ and the differential $d E$ at the point $(x, \xi)$ has nullity $m>0$. The focal set of $M$ is the set of all focal points of $(M, x)$ for all $x \in M$.

Since $E$ is a $C^{1}$ map and $N M$ and $\mathbb{S}^{n+k}$ have the same dimension, we can also assert that the focal set of $M$ has mesure zero in $\mathbb{S}^{n+k}$, due to Sard's Theorem. Thus, although $N M$ could have a large set of critical points, $M$ does not have so many focal points. Let us take $\xi$ a unit length normal vector to $f(M)$ at a point $x \in M$. The next theorem allows us to locate the focal points of $M$ at $x$ along the geodesic $E(x, t \xi)$, for $t \in \mathbb{R}$, in terms of the eigenvalues (principal curvatures) of the shape operator $A_{\xi}$ at $x$.

Theorem 1.37. Let $f: M^{n} \rightarrow \mathbb{S}^{n+k}$ be a submanifold of the sphere $\mathbb{S}^{n+k}$, and let $\xi$ be a unit normal vector to $f\left(M^{n}\right)$ at $f(x)$. Then $p=E(x, t \xi)$ is a focal point of $\left(M^{n}, x\right)$ of multiplicity $m>0$ if and only if there is an eigenvalue $\lambda$ of the shape operator $A_{\xi}$ of multiplicity $m$ such that $\lambda=\cot (t)$.

Proof. Since we are going to compute $d E$ at a point of $N M$ which is not in the zero section, we will consider that point to be of the form $E(x, t \xi) \in N M$, for $t>0$. Let $U$ a normal neighborhood $U$ of $x$ in $M$. Then $N U$ can be considered as $U \times \mathbb{R}^{k}$. Let an orthonormal 
frame $\left\{\xi_{1}, \ldots, \xi_{k}\right\}$ of normal vectors on $U$ at $x$ such that $\xi_{1}=\xi$, and extend them to orthonormal vector fields on $U$ by parallel transport with respect to the normal connection $\nabla^{\perp}$ along radial geodesics in $U$ through $x$. We now study the nullity of $d E$ at $(x, t \xi)$. Due to the trivial form of $N U$, we can parametrize it locally in a neighborhood of $(x, t \xi)$ as follows: Let $\left\{e_{1}, \ldots, e_{k}\right\}$ be the standard orthonormal basis of $\mathbb{R}^{k}$ and take the unit sphere in it given by

$$
\mathbb{S}^{k-1}=\left\{a=\sum_{j=1}^{k} a_{j} e_{j} \mid a_{1}^{2}+\cdots+a_{k}^{2}=1\right\} .
$$

Then the coordinate map for the normal bundle in a neighborhood of $(x, t \xi)$ can be written as

$$
\begin{aligned}
\Phi: U \times(0, \infty) \times \mathbb{S}^{k-1} & \longrightarrow N M \\
(y, \mu, a) & \longmapsto \Phi(y, \mu, a)=\mu \sum_{j=1}^{k} a_{j} \xi_{j}(y),
\end{aligned}
$$

where the vector $\Phi(y, \mu, a)$ is normal to $M$ at a point $y \in U$. Observe that the second coordinate represents the norm of a vector in the direction of a unit vector $a$, corresponding to the third coordinate. Thereby $(E \circ \Phi)(\mu, a, y)$ is the point in $\mathbb{S}^{n+k}$ reached by traversing a distance $\mu$ along the geodesic in $\mathbb{S}^{n+k}$ beginning at $y$ and having initial direction $\sum_{j=1}^{k} a_{j} \xi_{j}(y)$

$$
(E \circ \Phi)(y, \mu, a)=E\left(y, \mu \sum_{j=1}^{k} a_{j} \xi_{j}(y)\right)=\cos (\mu) y+\sin (\mu) \sum_{j=1}^{k} a_{j} \xi_{j}(y) .
$$

In this local parametrization, $(x, t \xi)$ is equal to $\Phi\left(x, t, e_{1}\right)$. Then, evaluating $d E$ at $(x, t \xi)$ is equivalent to computing $d(E \circ \Phi)$ at the point $\left(x, t, e_{1}\right)$. In this regard, a geodesic $\gamma$ is expressed by

$$
(E \circ \Phi)(x, t, \xi)=E(x, t \xi)=\exp _{x}(t \xi)=\gamma_{\xi}(t)=\gamma_{t \xi}(1)=\gamma(x, t, \xi)
$$

We now want to express $d(E \circ \Phi)$ at the point $\left(x, t, e_{1}\right)$ in terms of the following basis: an orthonormal basis of $T_{x} M$ consisting of eigenvectors $X$ of $A_{\xi}$ with corresponding eigenvalues denoted by $\lambda ; \frac{\partial}{\partial \mu}$, for $(0, \infty)$; and $\left\{e_{2}, \ldots, e_{k}\right\}$ for the tangent space $T_{e_{1}} \mathbb{S}^{k-1}$.

First, we compute the differential $d(E \circ \Phi)\left(\frac{\partial}{\partial \mu}\right)$ at $\left(x, t, e_{1}\right)$, obtaining

$$
\left.d(E \circ \Phi)\left(\frac{\partial}{\partial \mu}\right)\right|_{(x, t \xi)}=\left.\gamma^{\prime}(\mu)\right|_{\mu=t}
$$

where $\gamma^{\prime}(\mu)$ is the velocity vector to the curve $\gamma(\mu)$ is given by

$$
\gamma(\mu)=\cos (\mu) x+\sin (\mu) \xi_{1}(x),
$$

with $\xi=\xi_{1}(x)$ by hypothesis. Since $\gamma^{\prime}(\mu)=-\sin (\mu) x+\cos (\mu) \xi_{1}(x)$ and $\left\|\gamma^{\prime}(\mu)\right\|=1$, for all $\mu$, in particular the expression in eq. (1.17) is always different from zero. 
Similarly, for the orthonormal basis $\left\{e_{j}\right\}_{2 \leqslant j \leqslant k}$ of $T_{e_{1}} \mathbb{S}^{k-1}$, we compute $d(E \circ \Phi)\left(e_{j}\right)$. A curve lying in $\mathbb{S}^{k-1}$ with initial point $e_{1}$ and initial velocity vector $e_{j}$ has the form

$$
\alpha(s)=\cos (s) e_{1}+\sin (s) e_{j} .
$$

Thus, using eq. (1.16), we have that $d(E \circ \Phi)\left(e_{j}\right)$ is the initial velocity vector to the curve

$$
\gamma(s)=\cos (t) x+\sin (t)\left(\cos (s) \xi_{1}+\sin (s) \xi_{j}(x)\right) .
$$

Differentiating with respect to $s$ and substituting in $s=0$ - which represents $e_{1}=\alpha(0)$ in $\mathbb{S}^{k-1}$ - we get

$$
d(E \circ \Phi)\left(e_{j}\right)=\left.\gamma^{\prime}(s)\right|_{s=0}=\sin (t) \xi_{j}(x),
$$

that it is different from zero for $0<t<\pi$ and $\xi_{j}(x)$ is a unit vector.

It is important to point out that all the above calculations show that if

$$
V:=c_{1}\left(\frac{\partial}{\partial \mu}\right)+\sum_{j=2}^{k} c_{j} e_{j},
$$

then $d(E \circ \Phi)(V)=0$ only if $V=0$ i.e., when $c_{i}=0$ for all $i$.

It just left computing $d(E \circ \Phi) X$ for $X \in T_{x} M$. If $\beta(s)$ is a curve in $U$ with initial conditions $\beta(0)=x$ and $\beta^{\prime}(0)=X$, then $d(E \circ \Phi) X$ is the initial velocity vector to the curve

$$
\gamma(s)=(E \circ \Phi)\left(\beta(s), t, e_{1}\right)=\cos (t) \beta(s)+\sin (t) \xi_{1}(\beta(s)) .
$$

Differentiating with respect to $s$ and taking $s=0$, we get

$$
d(E \circ \Phi)(X)=\left.\gamma^{\prime}(s)\right|_{s=0}=\cos (t) X+\sin (t) \tilde{\nabla}_{X} \xi_{1} .
$$

From the decomposition in eq. (1.15), we know that $\tilde{\nabla}_{X} \xi_{1}=-A_{\xi_{1}} X+\nabla_{X}^{\perp} \xi_{1}$. Since, by hypothesis, the latter term $\nabla_{X}^{\perp} \xi_{1}=0$ and $\xi_{1}=\xi$, whence,

$$
d(E \circ \Phi)(X)=\left.\gamma^{\prime}(s)\right|_{s=0}=\cos (t) X-\sin (t) A_{\xi} X,
$$

where we are identifying $X$ with its parallel translate at the point $p=E(x, t \xi)$. The last equation shows that $d(E \circ \Phi)(X)=0$ if and only if

$$
A_{\xi} X=\cot (t) X
$$

or, equivalently, if $\cot (t)=: \lambda$ is eigenvalue of $A_{\xi}$ and $X$ its corresponding eigenvector. Hence, from eqs. (1.17), (1.19) and (1.22), a necessary and sufficient condition to the vanishing of $d(E \circ \Phi)(X+V)$ is that $V=0$ and eq. (1.23) holds. Therefore, if $\lambda=\cot (t)$ is an eigenvalue of multiplicity $m>0$ for $A_{\xi}$, then $m$ is both the dimension of the eigenspace $T_{\lambda}$ and the nullity of $d E$ at $(x, t \xi)$, completing the proof of this theorem.

When the codimension of $M^{n}$ in $\mathbb{S}^{n+k}$ is greater than one, we can define the notion of tube. Our motivation to consider that object is the form the leaves of the FKM-foliation are 
arranged in $\mathbb{S}^{n+k}$. We have two leaves that are focal manifolds, $M_{+}$and $M_{-}$, and the rest of the leaves constitute tubes over them. It is worth saying that all the following definitions and theorems are valid for any space form and the discussion is similar.

Definition 1.38. For $k>1$, we define the tube of radius $t>0$ over $M^{n}$ by the map

$$
\begin{aligned}
f_{t}: B M & \longrightarrow \mathbb{S}^{n+k} \\
(x, \xi) & \longmapsto f_{t}(x, \xi)=E(x, t \xi) .
\end{aligned}
$$

If $(x, t \xi)$ is not a critical point of $E$, then $f_{t}$ is an immersion in a neighborhood of $(x, \xi)$ in the unit normal bundle $B M$. From Theorem 1.37, it follows that for any point $x \in M$, there exists a neighborhood $U$ of $x$ in $M$ such that for all $t>0$ sufficiently small, the restriction of $f_{t}$ to the unit normal bundle $B U$ over $U$ is an immersion onto an $(n+k-1)$-dimensional manifold, which is geometrically a tube of radius $t$ over $U$.

In the case where $k=1$, then $M^{n}$ is a hypersurface of $\mathbb{S}^{n+k}$ and $B M$ is a double covering of $M$. In order to avoid this, we want to define $f_{t}$ over $M$ rather than over $B M$, which leads to the concept of a parallel hypersurface as below. In that circumstance, it is possible to assume for local computations that the hypersurface $M$ is orientable with a field of unit normal vectors denoted by $\xi$.

Definition 1.39. When $M$ is a hypersurface, i.e. $k=1$, we consider the parallel hypersurface

$$
\begin{aligned}
f_{t}: M & \longrightarrow \mathbb{S}^{n+k} \\
x & \longmapsto f_{t}(x)=E(x, t \xi),
\end{aligned}
$$

for $t \in \mathbb{R}$. For a negative value of $t$, the parallel hypersurface lies locally on the side of $M$ in the direction of the unit normal field $-\xi$, instead of on the side of $M$ in the direction of $\xi$. The original hypersurface $M$ corresponds to $t=0, f_{0}=f$.

We now proceed to give a formula for the shape operator of a tube over a submanifold of $\mathbb{S}^{n+k}$ in terms of its principal curvatures and vectors. Notice that if $k=1$, the case is reduced to the hypersurface $f_{t} M$ and then there are no terms $A_{t} e_{j}$. The notation at the beginning of the preceding proof remains unchangeable for this theorem, i.e., for a normal neighborhood $U$ of $x \in M$, we have a frame $\left\{\xi_{1}, \ldots, \xi_{k}\right\}$ of orthonormal vector fields constructed using parallel translation along radial geodesics as earlier, and such that $\xi_{1}=\xi$.

Theorem 1.40. Let $M^{n}$ be a submanifold of $\mathbb{S}^{n+k}$ and $\xi$ a unit normal vector to $M$ at $p$ such that $f_{t}: B M \rightarrow \mathbb{S}^{n+k}$ is an immersion at the point $(p, \xi) \in B M$. Let $\left\{X_{1}, \ldots, X_{n}\right\}$ be a basis of $T_{p} M$ consisting of principal vectors of $A_{\xi}$ with $A_{\xi} X_{i}=\lambda_{i} X_{i}$ for $1 \leqslant i \leqslant n$. The shape operator $A_{t}$ of the tube $f_{t}$ of radius $t$ over $M$ at the point $(x, \xi)$ is given in terms of its principal vectors as follows:

- for $2 \leqslant j \leqslant k, A_{t} e_{j}=-\cot (t) e_{j}$,

- for $1 \leqslant i \leqslant n, A_{t} X_{i}=\cot \left(\theta_{i}-t\right) X_{i}$, if $\lambda_{i}=\cot \left(\theta_{i}\right), 0<\theta_{i}<\pi$. 
Proof. We define the unit normal bundle $B M$ in local coordinates in a neighborhood of the point $(x, \xi)$ by the map

$$
\begin{aligned}
\Phi: U \times \mathbb{S}^{k-1} & \longrightarrow B M \\
(y, a) & \longmapsto \Phi(y, a)=\sum_{j=1}^{k} a_{j} \xi_{j}(y),
\end{aligned}
$$

where $\Phi(y, a)$ is a unit normal vector to $M$ at the point $y \in U$.

In this local parametrization, the point $x, \xi$ in $B M$ is equals to $\Phi\left(x, e_{1}\right)$, then evaluating $d f_{t}$ at $(x, \xi)$ is equivalent to evaluate $d\left(f_{t} \circ \Phi\right)$ at the point $\left(x, e_{1}\right)$.

Similarly to the proof in Theorem 1.37, we want to express $d\left(f_{t} \circ \Phi\right)$ at $\left(x, e_{1}\right)$ in terms of the following basis: an orthonormal basis of $T_{x} M$ consisting of eigenvectors $X$ of $A_{\xi}$ with corresponding eigenvalues denoted by $\lambda$; and $\left\{e_{2}, \ldots, e_{k}\right\}$ for the tangent space $T_{e_{1}} \mathbb{S}^{k-1}$. Analogously to the computations in eqs. (1.19) and (1.22),

$$
d\left(f_{t} \circ \Phi\right)\left(e_{j}\right)=\sin (t) \xi_{j}(x)
$$

and

$$
d\left(f_{t} \circ \Phi\right)(X)=\cos (t) X-\sin (t) A_{\xi} X,
$$

where we have identified $X$ with its parallel transport at the point $f_{t}(x, \xi)$.

Since $f_{t}$ is an immersion at $(x, \xi)$, there is a neighborhood $W$ of the point $(x, \xi)$ in the unit normal bundle $B U$ such that the restriction of $f_{t}$ to $W$ is an embedded hypersurface in $\mathbb{S}^{n+k}$. To find the shape operator of $f_{t} W$, we need to find a local field of unit normals to $f_{t} W$, and then compute its covariant derivative. To do so, we parallel transport the unit normal vector $\eta$ of a point $(w, \eta)$ to the hypersurface $f_{t} W$ at the point $f_{t}(w, \eta)$. Hence, we obtain a field of unit normals to $f_{t} W$ on the neighborhood $W$ of $B U$, denoted by $\eta$. We will denote the corresponding shape operator to the oriented hypersurface $f_{t} W$ by $A_{t}$.

As in the case of the normal bundle, we can identify the tangent space of $B M$ at $(x, \xi)$ with $T_{x} M \times T_{e_{1}} \mathbb{S}^{k-1}$ via the local parametrization $\Phi$. Besides, for $Z \in T_{x} M \times T_{e_{1}} \mathbb{S}^{k-1}$ we can write the shape operator $A_{t}$ by

$$
d\left(f_{t} \circ \Phi\right)\left(A_{t} Z\right)=-\tilde{\nabla}_{d\left(f_{t} \circ \Phi\right) Z} \eta
$$

where there is no term involving the normal connection $\nabla^{\perp}$ since the codimension of $f_{t} W$ is one. We now proceed to compute the shape operator for the $e_{j}$. As in eq. (1.18), we already know that $d\left(f_{t} \circ \Phi\right)\left(e_{j}\right)$ is the initial vector to the curve

$$
\gamma(s)=\cos (t) x+\sin (t)\left(\cos (s) \xi_{1}+\sin (s) \xi_{j}(x)\right) .
$$

Along $\gamma$, the unit normal field $\eta$ to the tube over $M$ is given by the derivative of that curve,

$$
\eta(s)=-\sin (t) x+\cos (t)\left(-\sin (s) \xi_{1}+\cos (s) \xi_{j}(x)\right) .
$$


Hence, $\tilde{\nabla}_{d\left(f_{t} \circ \Phi\right)\left(e_{j}\right)} \eta=\eta^{\prime}(0)$, where

$$
\eta^{\prime}(0)=\cos (t) \xi_{j}(x)
$$

and replacing in eq. (1.26) we obtain

$$
d\left(f_{t} \circ \Phi\right)\left(A_{t} e_{j}\right)=-\eta^{\prime}(0)=-\cos (t) \xi_{j}(x) .
$$

Besides, we can use eq. (1.24) in the equation below to get $A_{t} \sin (t) e_{j}=-\cos (t) e_{j}$ or, equivalently,

$$
A_{t} e_{j}=-\cot (t) e_{j}
$$

which means that $e_{j}$ is principal vector of $A_{t}$ with corresponding principal curvature $-\cot (t)$, for $t>0$ the radius of the tube.

Next we repeat the procedure above for $A_{t} X$ with $X \in T_{x} M$. Similar to eq. (1.21), let $\beta(s)$ be a curve in $M$ with initial conditions $\beta(0)=x$ and $\beta^{\prime}(0)=X$. The derivative $d\left(f_{t} \circ \Phi\right)(X)$ is the initial velocity vector to the curve

$$
\gamma(s)=\cos (t) \beta(s)+\sin (t) \xi_{1}(\beta(s))
$$

The unit normal field $\eta$ to the tube along $\gamma(s)$ is the velocity vector

$$
\eta(s)=-\sin (t) \beta(s)+\cos (t) \xi_{1}(\beta(s)),
$$

Furthermore, $\tilde{\nabla}_{d\left(f_{t} \circ \Phi\right)(X)} \eta$ is the initial velocity vector $\eta^{\prime}(0)$, where

$$
\eta^{\prime}(0)=-\sin (t) X+\cos (t) \tilde{\nabla}_{X} \xi_{1}(x)
$$

and we are identifying parallel vectors in $\mathbb{S}^{n+k}$. Thus it follows from eq. (1.15) and hypothesis $\nabla_{X}^{\perp} \xi=0$ and $\xi_{1}=\xi$ that

$$
\tilde{\nabla}_{d\left(f_{t} \circ \Phi\right)(X)} \eta=-\sin (t) X-\cos (t) A_{\xi} X
$$

Applying again eq. (1.26), we obtain a relation between both shape operators $A_{\xi}$ and $A_{t}$,

$$
d\left(f_{t} \circ \Phi\right)\left(A_{t} X\right)=\sin (t) X+\cos (t) A_{\xi} X
$$

We also know from eq. (1.25) that $d\left(f_{t} \circ \Phi\right)(X)=\left(\cos (t) I-\sin (t) A_{\xi}\right) X$, then

$$
\left(\cos (t) I-\sin (t) A_{\xi}\right) A_{t} X=\left(\sin (t) I+\cos (t) A_{\xi}\right) X
$$

In the case of a principal vector $X$ such that $A_{\xi} X=\lambda X$, the expression above leads to

$$
\begin{aligned}
A_{t} X & =\left(\cos (t) I-\sin (t) A_{\xi}\right)^{-1}\left(\sin (t) I+\cos (t) A_{\xi}\right) X \\
& =\left(\frac{\cos (t) \lambda+\sin (t)}{\cos (t)-\sin (t) \lambda}\right) X .
\end{aligned}
$$


If we express the principal curvatures of $X$ according to the preceeding theorem (see eq. (1.23)), then $\lambda=\cot (\theta)$, for $0<\theta<\pi$, thus substituting in the equation above we get

$$
\begin{aligned}
A_{t} X & =\left(\frac{\cos (t) \cot (\theta)+\sin (t)}{\cos (t)-\sin (t) \cot (\theta)}\right) X \\
& =\left(\frac{\cot (t) \cot (\theta)+1}{\cot (t)-\cot (\theta)}\right) X .
\end{aligned}
$$

This can be reduced to

$$
A_{t} X=\cot (\theta-t) X
$$

showing that $X$ is a principal vector of $A_{t}$ with corresponding principal curvature $\cot (\theta-t)$, and this finishes the proof.

As a consequence of Theorems 1.37 and 1.40 we obtain the following useful result describing the focal set of a parallel hypersurface when $M$ is a hypersurface in the sphere $\mathbb{S}^{n+k}$, and of the tube, when $M$ has codimension greater than one.

Theorem 1.41. Let $M^{n}$ be a submanifold of $\mathbb{S}^{n+k}$ and $t$ a real number such that $f_{t} M$ is a hypersurface.

(a) If $M$ is a hypersurface, then the focal set of the parallel hypersurface $f_{t} M$ is the focal set of $M$.

(b) If $M$ has codimension greater than one, thn the focal set of the tube $f_{t} M$ consists of the union of the focal set of $M$ with $M$ itself.

From this we can also conclude that all the elements in $M$ are focal points of the tube $f_{t} M$ corresponding to the principal curvature $\lambda=-\cot (t)$. Besides, since $\lambda_{i}=\cot \left(\theta_{i}\right)=$ $\cot \left(\theta_{i}-\pi\right)$, for any $x \in M$ each principal curvature gives rise to two antipodal focal points. In fact, Münzner showed that there are $2 g$ focal points, a pair for each $g$ distinct principal curvature, which are evenly spaced along a normal geodesic to the $f_{t} M$ parallel isoparametric hypersurfaces. Furthermore, regardless of the number $g$ distinct principal curvatures, there are only two focal submanifolds $M_{+}$and $M_{-}$- the focal sets together with a natural manifold structure - where those focal points lie alternately.

\section{Isoparametric hypersurfaces with $g=4$ principal curvatures: the FKM family}

In this last section it is defined the FKM family, which is a set of isoparametric submanifolds with four principal curvatures, given by the level sets of a function on $\mathbb{S}^{2 l-1}$ satisfying the so called differential equations of Cartan-Münzner. In addition, there are computed explicitly its $g=4$ distinct principal curvatures.

Münzner showed that a connected isoparametric hypersurface $M$ in $\mathbb{S}^{2 l-1}$ with $g$ distinct principal curvatures $\lambda_{i}=\cot \left(\theta_{i}\right), 0<\theta_{i}<\pi$, and respective multiplicities $m_{i}$, together with its parallel hypersurfaces and focal submanifolds are each contained in a level set of an isoparametric function that satisfies two famous differential equations which now we present 
in the next theorem. This connects explicitely the theory for tubes and parallel hypersurfaces we described in the preceding section with the study of isoparametric functions and their hypersurfaces. The same author also proved (see for example [CR15, Theorem 3.26 and Corollary 3.28]) that there at most two distinct multiplicities $m_{1}, m_{2}$ among the $m_{i}$ stated above, satisfying $m_{i+2}=m_{i}$ (subscripts $\left.\bmod g\right)$.

Theorem 1.42. Let $M \subseteq \mathbb{S}^{2 l-1} \subseteq \mathbb{R}^{2 l}$ be a connected isoparametric hypersurface with $g$ principal curvatures $\lambda_{i}=\cot \left(\theta_{i}\right), 0<\theta_{i}<\pi$, with respective multiplicities $m_{i}$. Then $M$ is an open subset of a level set of the restriction to $\mathbb{S}^{2 l-1}$ of a homogeneous polynomial $F$ on $\mathbb{R}^{2 l}$ of degree $g$ satisfying the differential equations,

$$
\begin{aligned}
\left|\operatorname{grad}^{E} F\right|^{2} & =g^{2} r^{2 g-2}, \\
\Delta^{E} F & =c r^{g-2},
\end{aligned}
$$

where $r=|x|$, and $c=g^{2}\left(m_{2}-m_{1}\right) / 2$,

In the literature, $F$ is called the Cartan-Münzner polynomial and eqs. (1.30) and (1.31) are referred to as Cartan-Münzner equations.

Let us call $F_{0}$ the restriction of $F$ to the sphere $\mathbb{S}^{n+1}$. It follows from Theorem 1.29 and $r=|x|=1$ that $F_{0}$ satisfies the differential equations

$$
\begin{aligned}
\left|\operatorname{grad}^{S} F_{0}\right|^{2} & =g^{2}\left(1-F_{0}^{2}\right) \\
\Delta^{S} F_{0} & =c-g(g+n) F,
\end{aligned}
$$

where $c$ is the value defined in the theorem above. Thus $F_{0}$ is an isoparametric function since both $\left|\operatorname{grad}^{S} F\right|^{2}$ and $\Delta^{S} F$ are functions of $F_{0}$ itself as in Definition 1.28.

We now state the construction of the FKM family in the next theorem as an application of the theory we have studied so far (see for example [FKM81, Theorem 4.1] or [Cec13, Theorem 4.39]). The function $F$ below, which appeared first in Example 1.27, depends only on the Clifford sphere $\mathbb{S}_{C}$ and not on the choice of orthonormal basis $\left\{P_{0}, \ldots, P_{m}\right\}$, by Theorem 1.26 (c).

Theorem 1.43. Let $\left(P_{0}, \ldots, P_{m}\right)$ be a Clifford system on $\mathbb{R}^{2 l}$. Let $m_{1}=m, m_{2}=l-m-1$, and $F: \mathbb{R}^{2 l} \rightarrow \mathbb{R}$ be defined by

$$
F(x)=\langle x, x\rangle^{2}-2 \sum_{i=0}^{m}\left\langle P_{i} x, x\right\rangle^{2} .
$$

Then $F$ satisfies the Cartan-Münzner differential equations. If $m_{2}>0$, then the level sets of $F$ on $\mathbb{S}^{2 l-1}$ form a family of isoparametric hypersurfaces with $g=4$ principal curvatures with multiplicities $\left(m_{1}, m_{2}\right)$ called the FKM-family.

We finish this section by providing the explicit form of the principal curvatures of the isoparametric hypersurfaces in the FKM family. To do so, it is enough finding the principal curvatures of the focal manifold $M_{+}$, which in fact it is well known in the literature as a 
Clifford-Stiefel manifold (for a further geometric information contained in $M_{+}$and $M_{-}$see [FKM81, Section 4.2], [CR15, p. 174-175] and the proof of Proposition 2.2) and is defined by the preimage of the value -1 in the restriction $F_{0}=\left.F\right|_{\mathbb{S}^{n}}$ of its associated isoparametric function $F: M^{n+1} \rightarrow \mathbb{R}$, and then using Theorem 1.40. The first part is solved in the theorem below, whose proof is detailed in [Cec13, p. 109-111].

We keep the notation from Section 1.2, namely, the unit sphere $\mathbb{S}_{C}$ is the $\operatorname{span}\left\{P_{0}, \ldots, P_{m}\right\}$ on $\mathbb{R}^{m+1}$, and $E_{+}(P)$ and $E_{-}(P)$ are the $l$-dimensional eigenspaces for the eigenvalues -1 and -1 , respectively, so that $\mathbb{R}^{2 l}=E_{+}(P) \oplus E_{-}(P)$.

Theorem 1.44. Let $x$ be a point on the focal submanifold $M_{+}$, and let $\xi=P x$ be a unit normal vector to $M_{+}$at $x$, where $P \in \mathbb{S}_{C}$. Then the shape operator $A_{\xi}$ has principal curvatures $0,1,-1$ with corresponding principal spaces $T_{0}(\xi), T_{1}(\xi), T_{-1}(\xi)$ as follows:

$$
\begin{aligned}
T_{0}(\xi) & =\left\{Q P x \mid Q \in \mathbb{S}_{C},\langle Q, P\rangle=0\right\}, \\
T_{1}(\xi) & =\left\{X \in E_{-}(P) \mid X \cdot Q x=0, \forall Q \in \mathbb{S}_{C}\right\}=E_{-}(P) \cap T_{x} M_{+}, \\
T_{-1}(\xi) & =\left\{X \in E_{+}(P) \mid X \cdot Q x=0, \forall Q \in \mathbb{S}_{C}\right\}=E_{+}(P) \cap T_{x} M_{-} .
\end{aligned}
$$

Furthermore,

$$
\operatorname{dim} T_{0}(\xi)=m, \quad \operatorname{dim} T_{1}(\xi)=\operatorname{dim} T_{-1}(\xi)=l-m-1
$$

Combining the preceding theorem and Theorem 1.40, the following result exhibits the values for the $g=4$ distinct principal curvatures of the FKM family, which are tube hypersurfaces over its focal submanifold $M_{+}$. Notice also the constancy in such principal curvatures, which proves that these hypersurfaces form an isoparametric family in $\mathbb{S}^{2 l-1}$.

Corollary 1.45. Let $M_{t}$ be a tube of spherical radius $t$ over the focal submanifold $M_{+}$, where $0<t<\pi$ and $t \notin\left\{\frac{\pi}{4}, \frac{\pi}{2}, \frac{3 \pi}{4}\right\}$. Then $M_{t}$ is an isoparametric hypersurface with four distinct principal curvatures,

$$
\cot (-t), \quad \cot \left(\frac{\pi}{4}-t\right), \quad \cot \left(\frac{\pi}{2}-t\right), \quad \cot \left(\frac{3 \pi}{4}-t\right),
$$

having respective multiplicities $m, l-m-1, m, l-m-1$.

Proof. Each of the three principal curvatures found in Theorem 1.44 correspond to $\lambda_{i}=$ $\cot \left(\theta_{i}\right)$, for $1 \leqslant i \leqslant 3$ and $0<\theta_{i}<\pi$ of the equation $A_{t} X_{i}=\cot \left(\theta_{i}-t\right) X_{i}$ in Theorem 1.40. Then, for $\lambda_{1}=0$, we have $\cot \left(\theta_{1}\right)=0$, that implies $\theta_{1}=\frac{\pi}{2}$. Thus $\cot \left(\frac{\pi}{2}-t\right)$ is a principal curvature for $f_{t} M$ with multiplicity $m$. Analogously, for $\lambda_{2}=-1$, the principal curvature is $\cot \left(\frac{3 \pi}{4}-t\right)$ with multiplicity $l-m-1$; for $\lambda_{3}=1$ the value is $\cot \left(\frac{\pi}{4}-t\right)$ having multiplicity $l-m-1$ as well. Finally, it follows from equation $A_{t} e_{j}=-\cot (t) e_{j}$ also in Theorem 1.40 that $-\cot (t)=\cot (-t)$ where the multiplicity is equal to the dimension of the basis for the unit normals to $M_{+}$at $x$, that is, $m$. 


\section{Chapter 2}

\section{Examples of singular Riemannian foliations of higher codimension}

In this chapter it is studied the construction, given by Radeschi in [Rad14] of the recent examples of singular Riemannian foliations of higher codimension. We begin with Clifford foliations in section 2.1, then composed foliations in section 2.2 and close with a beautiful correspondence between SRF and Clifford systems in section 2.3.

\subsection{Clifford foliations}

Let $C$ be a Clifford system of rank $m+1$ on $\mathbb{R}^{2 l}, l=k \delta(m)$. On the unit sphere $\mathbb{S}^{2 l-1} \subset \mathbb{R}^{2 l}$ endowed with the canonical inner product which we also denote by $\langle\cdot, \cdot\rangle$, consider the function

$$
\pi_{C}: \mathbb{S}^{2 l-1} \longrightarrow \mathbb{R}_{C}=\mathbb{R}^{m+1}
$$

that takes $x \in \mathbb{S}^{2 l-1}$ to the unique element $\pi_{C}(x) \in \mathbb{R}_{C}$ defined by the property

$$
\left\langle\pi_{C}(x), P\right\rangle=\langle P x, x\rangle, \quad \forall P \in \mathbb{R}_{C}
$$

Fixing an orthonormal basis $\left(P_{0}, \ldots, P_{m}\right)$ of $\mathbb{R}_{C}$, the map $\pi_{C}$ can be rewritten as

$$
\pi_{C}(x)=\left(\left\langle P_{0} x, x\right\rangle, \ldots,\left\langle P_{m} x, x\right\rangle\right)=\sum_{i=0}^{m}\left\langle P_{i} x, x\right\rangle P_{i} .
$$

Lemma 2.1. The image of $\pi_{C}$ is contained in the unit disk $\mathbb{D}_{C}$ of $\mathbb{R}_{C}$.

Proof. Let $x \in \mathbb{S}^{2 l-1}$ and $P=\pi_{C}(x)$. It is enough to show that $\|P\| \leqslant 1$. The case $P=0_{2 l}$ is trivial. Otherwise, by the Cauchy-Schwarz inequality we have

$$
\|P\|^{2}=\langle P, P\rangle=\left\langle\pi_{C}(x), P\right\rangle=\langle P x, x\rangle \leqslant\|P x\|\|x\|=\|P\|\|x\|^{2}=\|P\| .
$$

Then, $\|P\|^{2} \leqslant\|P\|$ implies $\|P\| \leqslant 1$.

From now on, the disk $\mathbb{D}_{C}$ is endowed with a (hemisphere) metric of constant sectional curvature 4 , that has a totally geodesic boundary $\partial \mathbb{D}_{C}=\mathbb{S}_{C}$; in that way $\mathbb{D}_{C}$ can be seen as the upper half sphere of radius $\frac{1}{2}$. 
Proposition 2.2. Given a Clifford system $C$ of rank $m+1$ on $\mathbb{R}^{2 l}, l=k \delta(m)$, the corresponding map

$$
\pi_{C}: \mathbb{S}^{2 l-1} \longrightarrow \mathbb{D}_{C}
$$

satisfies:

(1) The preimage of $P \in \mathbb{S}_{C}=\partial \mathbb{D}_{C}$ is the unit sphere $E_{+}^{1}(P)$ in the positive eigenspace $E_{+}(P)$. Moreover, the restriction $\left.\pi_{C}\right|_{M_{-}}: M_{-} \longrightarrow \mathbb{S}_{C}$ to the set $M_{-}:=\pi_{C}^{-1}\left(\mathbb{S}_{C}\right)$ is a submersion.

(2) If $l=m$, the image of $\pi_{C}$ is $\mathbb{S}_{C}$.

(3) If $l \geqslant m+1$, the map $\pi_{C}$ is surjective onto $\mathbb{D}_{C}$ and its restriction to $\mathbb{S}^{2 l-1} \backslash M_{-}$- the regular part-is a submersion.

(4) If $l>m+1$, the fibers of $\pi_{C}$ are connected.

(5) If $l=m+1, C$ can be extended to a Clifford system $C^{\prime}$ of rank $m+2$, the image of $\pi_{C^{\prime}}$ is $\mathbb{S}_{C^{\prime}}=\mathbb{S}^{m+1}$ and $\pi_{C}$ factors as $\pi_{C}=\operatorname{Pr} \circ \pi_{C^{\prime}}$, where $\operatorname{Pr}: \mathbb{S}_{C^{\prime}} \rightarrow \mathbb{D}_{C}$ is the projection of the first $m$ components, $\operatorname{Pr}\left(x_{1}, \ldots, x_{m}, x_{m+1}\right)=\left(x_{1}, \ldots, x_{m}\right)$. In particular, the fibers of $\pi_{C}$ are disconnected.

Proof. (1) Let $x \in \mathbb{S}^{2 l-1}$ and $P=\pi_{C}(x)$. If $P$ lies in $\mathbb{S}_{C}$ then $\|P\|=1$, in particular, $\|P\|^{2}=\|P\|$. Thus the inequality in eq. (2.2) is actually an equality and we get $\langle P x, x\rangle=\|P\| \cdot\|x\|^{2}=1$. Thus, $P x=x$, so $x$ is a unit eigenvector for $P$, with eigenvalue $\lambda=1$. We have proved that $\pi_{C}^{-1}(P) \subseteq E_{+}^{1}(P)$.

Consider now $x \in E_{+}^{1}(P)$, for $x \in \mathbb{S}^{2 l-1}$. From the definition in eq. (2.1) and using the Cauchy-Schwarz inequality,

$$
\left\|\pi_{C}(x)\right\| \geqslant\left\|\pi_{C}(x)\right\|\|P\| \geqslant\left\langle\pi_{C}(x), P\right\rangle=\langle P x, x\rangle=\langle x, x\rangle=1 .
$$

By eq. (2.1), $\pi_{C}(x) \in \mathbb{D}_{C}$ then, in the inequality above, $\left\|\pi_{C}(x)\right\|=1$ and therefore $P=\pi_{C}(x)$, showing that $E_{+}^{1}(P)$ is contained in $\pi_{C}^{-1}(P)$. Hence, the focal manifold $M_{-}:=\pi_{C}^{-1}\left(\mathbb{S}_{C}\right)$ embeds in $\mathbb{S}^{2 l-1} \times \mathbb{S}_{C}$ as the set

$$
M_{-}=\left\{(x, P) \in \mathbb{S}^{2 l-1} \times \mathbb{S}_{C} \mid x \in E_{+}^{1}(P)\right\}
$$

of codimension $l-m$ in $\mathbb{S}^{2 l-1}$, and $\pi_{C}$ is just the projection onto the second factor, which is a submersion.

(2) Fix an orthonormal basis $\left(P_{0}, \ldots, P_{m}\right)$ for $\mathbb{R}_{C}$. Given $x \in \mathbb{S}^{2 m-1}$, let $x=a x_{+}+b x_{-}$ where $x_{ \pm} \in E_{ \pm}\left(P_{0}\right)$ are unit vectors, and $a^{2}+b^{2}=1$. We want to prove that, if $\pi_{C}(x)=P$, then

$$
\|P\|=\sum_{i=0}^{m}\left\langle P_{i} x, x\right\rangle^{2}=1
$$


For $i=0$ we obtain

$$
\begin{aligned}
\left\langle P_{0} x, x\right\rangle & =\left\langle P_{0}\left(a x_{+}\right), a x_{+}\right\rangle+\left\langle P_{0}\left(a x_{+}\right), b x_{-}\right\rangle+\left\langle P_{0}\left(b x_{-}\right), a x_{+}\right\rangle+\left\langle P_{0}\left(b x_{-}\right), b x_{-}\right\rangle \\
& =\left\langle a x_{+}-b x_{-}, a x_{+}+b x_{-}\right\rangle \\
& =a^{2}\left\|x_{+}\right\|^{2}-b\left\|x_{-}\right\|^{2} \\
& =a^{2}-b^{2}
\end{aligned}
$$

where we have used in the second line that $P_{0} x_{+}=x_{+}, P_{0} x_{-}=-x_{-}$and $x_{+} \perp x_{-}$. For $1 \leqslant i \leqslant m$,

$$
\begin{aligned}
\left\langle P_{i} x, x\right\rangle & =a^{2}\left\langle P_{i} x_{+}, x_{+}\right\rangle+a b\left\langle P_{i} x_{+}, x_{-}\right\rangle+a b\left\langle P_{i} x_{-}, x_{+}\right\rangle+b^{2}\left\langle P_{i} x_{-}, x_{-}\right\rangle \\
& =2 a b\left\langle P_{i} x_{+}, x_{-}\right\rangle,
\end{aligned}
$$

where we have used the following facts: $\left\langle P_{i} x_{-}, x_{+}\right\rangle=\left\langle x_{-}, P_{i}^{T} x_{+}\right\rangle=\left\langle x_{-}, P_{i} x_{+}\right\rangle$since $P_{i}$ is symmetric, $P_{i} x_{ \pm} \in E_{\mp}\left(P_{0}\right)$ and $P_{i} x_{ \pm} \perp x_{ \pm}$. In addition, it follows from the symmetry of $P_{i}$ and the anticommutativity for $P_{i} P_{j}$ whenever $i \neq j$ that

$$
\left\langle P_{i} x_{+}, P_{j} x_{+}\right\rangle=\left\langle P_{j} P_{i} x_{+}, x_{+}\right\rangle=\left\langle-P_{i} P_{j} x_{+}, x_{+}\right\rangle=\left\langle x_{+},-P_{j} P_{i} x_{+}\right\rangle .
$$

After adding the second and fourth expressions in eq. (2.4) we get $2\left\langle P_{i} x_{+}, P_{j} x_{+}\right\rangle=0$, whence $P_{i} x_{+}$and $P_{j} x_{+}$are orthogonal. Each $P_{i} x_{+}$is also a unit vector since

$$
\left\langle P_{i} x_{+}, P_{i} x_{+}\right\rangle=\left\langle P_{i}^{2} x_{+}, x_{+}\right\rangle=\left\langle x_{+}, x_{+}\right\rangle=1 .
$$

Because $l=m=\operatorname{dim} E_{-}\left(P_{0}\right)$, we conclude from above that $P_{1} x_{+}, \ldots, P_{m} x_{+}$form an orthonormal basis of $E_{-}\left(P_{0}\right)$ and consequently

$$
\begin{aligned}
\|P\|^{2}=\sum_{i=0}^{m}\left\langle P_{i} x, x\right\rangle^{2} & =\left\langle P_{0} x, x\right\rangle^{2}+4 a^{2} b^{2} \sum_{i=1}^{m}\left\langle P_{i} x_{+}, x_{-}\right\rangle^{2} \\
& =\left(a^{2}-b^{2}\right)^{2}+4 a^{2} b^{2}=\left(a^{2}+b^{2}\right)^{2} \\
& =1,
\end{aligned}
$$

where the third equality follows from the norm of $x_{-}$written in the $\left\{P_{i} x_{+}\right\}_{i=1}^{m}$ basis, i.e., $\left\|x_{-}\right\|^{2}=\sum_{i=1}^{m}\left\langle P_{i} x_{+}, x_{-}\right\rangle^{2}=1$.

Further, as we already noted in (1) the preimage of any $P \in \mathbb{S}_{C}$ is non empty, since it consists of the unit sphere $E_{+}^{1}(P)$, and then the image of $\pi_{C}$ is $\mathbb{S}_{C}$.

(3) Fix an orthonormal basis $\left(P_{0}, \ldots, P_{m}\right)$ for $\mathbb{R}_{C}$. In order to prove the surjectivity of $\pi_{C}$ onto $\mathbb{D}_{C}$, let us find first the preimage of 0 under this map. If there exists $x$ such that $\pi_{C}(x)=\sum_{i=0}^{m}\left\langle P_{i} x, x\right\rangle P_{i}=0$, then all the coefficients $\left\langle P_{i} x, x\right\rangle$ should vanish for all $i$, due to $\left\{P_{i}\right\}_{i}$ being a basis. This implies the set $\left\{P_{0} x, \ldots, P_{m} x\right\}$ must be orthogonal to $x$. Since $\mathbb{S}^{2 l-1} \subseteq E_{+}\left(P_{0}\right) \oplus E_{-}\left(P_{0}\right)$, we can express $x$ as $a x_{+}+b x_{-}$, for $x_{ \pm} \in E_{ \pm}^{1}\left(P_{0}\right)$ and $a, b$ real numbers such that $a^{2}+b^{2}=1$. Note that neither $a$ nor $b$ can be zero because both situations contradict $x$ being perpendicular to $P_{0} x$. 
Returning to the condition $\left\langle P_{i} x, x\right\rangle=0$, and by the same computations as in item (2), we have

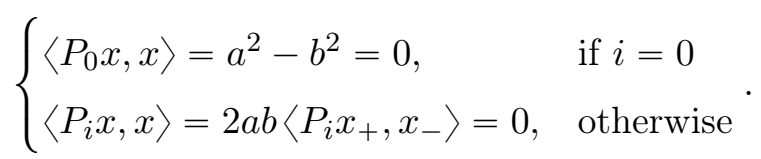

It follows from the first case that $a= \pm b$. Moreover $a= \pm \frac{\sqrt{2}}{2}$ because $a^{2}+b^{2}=1$. However, it is enough considering $a=b=\frac{\sqrt{2}}{2}$ since $\pm \frac{\sqrt{2}}{2} x_{+} \in E_{+}^{1}\left(P_{0}\right)$ as well as $\pm \frac{\sqrt{2}}{2} x_{-} \in E_{-}^{1}\left(P_{0}\right)$. Then we deduce from the second equation that $\left\langle P_{i} x_{+}, x_{-}\right\rangle=0$, in other words, $P_{i} x_{+}$must lie in $E_{-}\left(P_{0}\right)$ for $i \neq 0$-as $P_{i}$ interchanges the eigenspaces of $P_{0}$ - and $x_{-}$should be in the orthogonal complement of $\operatorname{span}\left\{P_{1} x_{+}, \ldots, P_{m} x_{+}\right\}$ inside $E_{-}\left(P_{0}\right)$, where we are bearing in mind from hypothesis that this last space has dimension $l \geqslant m+1$. Summarizing, the element form of $x$ in $\mathbb{S}^{2 l-1}$, such that $\pi_{C}(x)=0$, must be $\frac{\sqrt{2}}{2}\left(x_{+}+x_{-}\right)$. As a matter of fact we claim that

$$
M_{+}:=\pi_{C}^{-1}(0)=\left\{\frac{\sqrt{2}}{2}\left(x_{+}+x_{-}\right): x_{ \pm} \in E_{ \pm}^{1}\left(P_{0}\right), x_{-} \perp \operatorname{span}\left\{P_{1} x_{+}, \ldots, P_{m} x_{+}\right\}\right\} .
$$

To see this, it just remains computing the image of $x=\frac{\sqrt{2}}{2}\left(x_{+}+x_{-}\right)$in $\mathbb{S}^{2 l-1}$ under that map:

$$
\begin{aligned}
\pi_{C}(x) & =\sum_{i=0}^{m}\left\langle P_{i} x, x\right\rangle P_{i} \\
& =\frac{1}{2}\left\langle P_{0} x_{+}+P_{0} x_{-}, x_{+}+x_{-}\right\rangle P_{0}+\frac{1}{2} \sum_{i=1}^{m}\left\langle P_{i} x_{+}+P_{i} x_{-}, x_{+}+x_{-}\right\rangle P_{i} \\
& =\frac{1}{2}\left\langle x_{+}-x_{-}, x_{+}+x_{-}\right\rangle P_{0}+\frac{1}{2} \sum_{i=1}^{m}\left(\left\langle P_{i} x_{+}, x_{-}\right\rangle P_{i}+\left\langle P_{i} x_{-}, x_{+}\right\rangle P_{i}\right) \\
& =\frac{1}{2}\left(\left\|x_{+}\right\|^{2}-\left\|x_{-}\right\|^{2}\right)+\sum_{i=1}^{m}\left\langle P_{i} x_{+}, x_{-}\right\rangle P_{i} \\
& =0 .
\end{aligned}
$$

From this it also follows that the focal manifold $M_{+}$is non empty. Notice that $M_{+}$ has codimension $m$ since $\operatorname{dim} E_{ \pm}$

Observe that any point in $\mathbb{D}_{C}$ can be expressed as $\sin (2 t) Q$, with $Q \in \mathbb{S}_{C}$ and $t \in\left[0, \frac{\pi}{4}\right]$. It can be shown that not only the fiber under $\pi_{C}$ of each element $\sin (2 t) Q$ is the set

$$
M_{(Q, t)}:=\left\{\cos (t) x+\sin (t) Q x \mid x \in M_{+}\right\},
$$

but also that map is surjective onto $\mathbb{D}_{C}$, as we show as follows:

$$
\begin{aligned}
\pi_{C}(\cos (t) x+\sin (t) Q x)= & \cos ^{2}(t) \sum_{i=0}^{m}\left\langle P_{i} x, x\right\rangle P_{i}+\cos (t) \sin (t) \sum_{i=0}^{m}\left\langle P_{i} x, Q x\right\rangle P_{i} \\
& +\cos (t) \sin (t) \sum_{i=0}^{m}\left\langle P_{i} Q x, x\right\rangle P_{i}+\sin ^{2}(t) \sum_{i=0}^{m}\left\langle P_{i} Q x, Q x\right\rangle P_{i}
\end{aligned}
$$


The first term on the right hand side of eq. (2.6) is zero because $\pi_{C}(x)=0$. For the second term note that

$$
\sum_{i=0}^{m}\left\langle P_{i} x, Q x\right\rangle P_{i}=\sum_{i=0}^{m}\left\langle P_{i}, Q\right\rangle P_{i}\|x\|^{2}=Q .
$$

Using the symmetry of $P_{i}$ in the third one, we have $\sum_{i=0}^{m}\left\langle P_{i} Q x, x\right\rangle P_{i}=\sum_{i=0}^{m}\left\langle Q x, P_{i} x\right\rangle P_{i}$. Lastly we use eq. (1.6) and $Q^{T} Q=Q^{2}=I$ in the fourth term to get

$$
\begin{aligned}
\sum_{i=0}^{m}\left\langle P_{i} Q x, Q x\right\rangle P_{i} & =\sum_{i=0}^{m}\left\langle-Q P_{i} x, Q x\right\rangle P_{i}+2 \sum_{i=0}^{m}\left\langle P_{i}, Q\right\rangle\langle I x, Q x\rangle P_{i} \\
& =-\sum_{i=0}^{m}\left\langle P_{i} x, x\right\rangle P_{i}+2 \sum_{i=0}^{m}\left\langle P_{i}, Q\right\rangle\langle Q x, x\rangle P_{i} \\
& =-\pi_{C}(x)+2 \sum_{i=0}^{m}\left\langle P_{i}, Q\right\rangle\left\langle\pi_{C}(x), Q\right\rangle P_{i}=0 .
\end{aligned}
$$

By putting all this together in eq. (2.7) we finally obtain $\pi_{C}(\cos (t) x+\sin (t) Q x)=$ $\sin (2 t) Q$. Moreover, for $t$ and $Q$ fixed, the set $M_{(Q, t)}$ has codimension $m$ in $\mathbb{S}^{2 l-1}$ and thus it is the fiber corresponding to the point $\sin (2 t) Q$.

It just remains to be proved that the restriction of $\pi_{C}$ to its regular part is a submersion. Observe that for any $P \in \mathbb{S}_{C}$ the gradient of the function

$$
\begin{aligned}
f: \mathbb{S}^{2 l-1} & \longrightarrow \mathbb{R} \\
x & \longmapsto\langle P x, x\rangle
\end{aligned}
$$

in $\mathbb{S}^{2 l-1}$ is

$$
\begin{aligned}
X_{P}(x):=(\nabla f)_{T}(x) & =(\nabla f)(x)-(\nabla f)_{N}(x) \\
& =(\nabla f)(x)-\langle(\nabla f)(x), \vec{N}\rangle \vec{N} \\
& =2 P x-2\langle P x, x\rangle x,
\end{aligned}
$$

where the normal vector to the point $x$ on a unit sphere is $\vec{N}=\frac{x}{\|x\|}=x$ and

$$
(\nabla f)(x)=\langle P I, x\rangle+\langle P x, I\rangle=P x+P x=2 P x .
$$

Now let us see that the $X_{P_{i}}(x)$ form a basis for a $m+1$-dimensional subspace of $T_{x} \mathbb{S}^{2 l-1}$ orthogonal to the fibers of $\pi_{C}$, when $x$ projects to the interior of $\mathbb{D}_{C}$. First note that $X_{P_{i}}(x) \neq 0$, otherwise there exists $i$ such that $P_{i} x=\left\langle P_{i} x, x\right\rangle x$ implying $x$ belongs to $E_{\lambda}^{1}\left(P_{i}\right)$, where $\lambda:=\left\langle P_{i} x, x\right\rangle$ and $\lambda$ cannot be zero. In fact, $P_{i}$ just have two eigenspaces, so $\lambda= \pm 1$. However $x \notin E_{+}^{1}\left(P_{i}\right)$ because $x$ does not project to $\mathbb{S}_{C}$. Therefore $x$ must lies in the negative eigenspace, thus $P_{i} x=-x$. There arises a contradiction since $\left\|\pi_{C}(-x)\right\|^{2}=\left\|\pi_{C}(x)\right\|^{2}<1$, whereas

$$
\left\|\pi_{C}\left(P_{i} x\right)\right\|^{2}=\left\langle P_{i} x, x\right\rangle^{2}+\sum_{j \neq i}\left\langle P_{j} x, x\right\rangle^{2} \geqslant 1 ;
$$


thus $X_{P_{i}}(x)$ is different from zero for all $i$. Now we are going to show that $X_{P_{i}}(x)$ are linearly independent. If a set $\left\{u_{0}, \ldots, u_{m}\right\}$ is linearly independent, then so does $\left\{u_{0}-\lambda_{0} v, \ldots, u_{m}-\lambda_{m} v\right\}$, for any nonzero vector $v$ and nonzero constants $\lambda_{i}$ as well. The desired result is obtained taking $u_{i}=P_{i} x, \lambda_{i}=\left\langle P_{i} x, x\right\rangle$ and $v=x$; hence the set of $X_{P_{i}}(x)$ spans a subspace of $T_{x} \mathbb{S}^{2 l-1}$ of dimension $m+1$ orthogonal to the fibers of $\pi_{C}$, thus projecting onto $T_{\pi_{C}(x)} \mathbb{D}_{C}$, and consequently $\pi_{C}$ is a submersion in $\mathbb{S}^{2 l-1} \backslash M_{-}$.

(4) Again, take an orthonormal basis $\left(P_{0}, \ldots, P_{m}\right)$ for $\mathbb{R}_{C}$ and $x_{+} \in E_{+}^{1}\left(P_{0}\right)$. On $E_{-}\left(P_{0}\right)$, consider the orthogonal complement $V_{x_{+}}$of $\operatorname{span}\left(P_{1} x_{+}, \ldots, P_{m} x_{+}\right)$and take its unit sphere $V_{x_{+}}^{1} \in \mathbb{S}^{2 l-1}$ which has dimension $l-m-1$. We have already checked that $\pi_{C}(x)=0$ when $x=\frac{\sqrt{2}}{2}\left(x_{+}+x_{-}\right) \in M_{+}$and $x_{-} \in V_{x_{+}}^{1}$. Taking the union of all $V_{x_{+}}^{1}$ as $x_{+}$varies in $E_{+}^{1}\left(P_{0}\right)$, we obtain a sphere bundle $V^{1} \rightarrow E_{+}^{1}\left(P_{0}\right)$ whose fiber has dimension $l-m-1$. In particular, if $l>m+1$ the fiber is connected, and so is $V^{1}$, since the connectedness of both the fibers and the base space under a surjective submersion - as it is the case of the sphere bundle - implies the connectedness of the total space (we will give a proof of it in Proposition 2.3). Furthermore, $M_{+}$is connected as well because this property is preserved under the continuous, surjective map $V^{1} \rightarrow M_{+}$, which sends $y \in V_{x}^{1}$ to $\frac{\sqrt{2}}{2}(x+y)$. Lastly, since all the fibers $M_{(Q, t)}$ of points in the interior of $\mathbb{D}_{C}$ are homeomorphic to each other, and in particular to $M_{+}=M_{(Q, 0)}$, every fiber of $\pi_{C}$ is connected.

(5) Observe that given a Clifford system of rank $m^{\prime}+1=(m+1)+1$ on $\mathbb{R}^{2 l}$ and $l=m+1$ we obtain a map $\pi_{C^{\prime}}$ whose image if $\mathbb{S}_{C^{\prime}}=\mathbb{S}^{m+1}$ because we are in the case of part (2). Moreover, since $l=m+1$, it follows from Table 1.1 that $m=3,7$, which are not multiples of 4 . Given a Clifford system $C^{\prime}$ of rank $m+2$ in $\mathbb{R}^{2 l}$, by the uniqueness of Clifford systems for $m \not \equiv 0(\bmod 4)$ it follows that $C$ is algebraically (and geometrically) equivalent to a sub-Clifford system of $C^{\prime}$. We can thus find an orthonormal basis $\left(P_{0}, \ldots, P_{m+1}\right)$ of $\mathbb{R}_{C^{\prime}}$ such that $\left(P_{0}, \ldots, P_{m}\right)$ is a basis for $\mathbb{R}_{C}$. Since we can express $\pi_{C}(x)$ as $\left(\left\langle P_{0}, x\right\rangle, \ldots,\left\langle P_{m}, x\right\rangle\right)$ and similarly for $\pi_{C^{\prime}}, \pi_{C}$ factors as $\pi_{C}=\operatorname{Pr} \circ \pi_{C^{\prime}}$, where $\operatorname{Pr}: \mathbb{S}_{C^{\prime}} \rightarrow \mathbb{D}_{C}$ is given by $\operatorname{Pr}\left(x_{1}, \ldots, x_{m}, x_{m+1}\right)=\left(x_{1}, \ldots, x_{m}\right)$.

In part (4) of this proposition, it remained the proof about the connectedness of the total space under a surjective submersion that is now given below.

Proposition 2.3. Let $f: M \rightarrow N$ a surjective submersion between two smooth manifolds. If both $N$ and all the fibers of $f$ are connected, then $M$ is connected as well.

Proof. Suppose we are given a continuous function $g: M \rightarrow\{0,1\}$. Connectedness is preserved under continuous maps, thus $g$ is constant on every fiber of $f$. Furthermore, since $f: M \rightarrow N$ is a quotient map (it is open and surjective), $g$ descends to a continuous map 
$\tilde{g}: N \rightarrow\{0,1\}$ such that $\tilde{g} \circ f=g$, as shown in the diagram below.

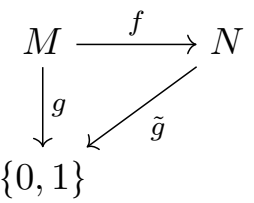

As $N$ is connected, $\tilde{g}$ (and hence also $g$ ) must be constant. We have shown that every continuous map from $M$ to $\{0,1\}$ is constant, which is equivalent to the connectedness of $M$.

Remark 2.4. If $C$ and $C^{\prime}$ are algebraically equivalent Clifford systems, then by the definition given in Section 1.2, there exists an orthogonal map $A \in \mathrm{O}(2 l)$ such that $\pi_{C^{\prime}}=A^{-1} \circ \pi_{C} \circ A$. In particular, up to orthogonal transformation, $\pi_{C}$ only depends on the algebraic equivalence class of $C$. It turns out that the converse is true, namely, the geometric equivalence class of $C$ is uniquely determined by $\pi_{C}$. This will be proved in Proposition 2.19.

Remark 2.5. Since we are interested in having connected fibers, from now on we will not consider Clifford systems with $l=m+1$.

Proposition 2.6. Let $C$ be a Clifford system of rank $m+1$ on $\mathbb{R}^{2 l}$. The fibers of $\pi_{C}$ define a transnormal system on $\mathbb{S}^{2 l-1}$, whose leaf space is $\mathbb{D}_{C}($ if $l>m+1)$ or $\mathbb{S}_{C}($ if $l=m$ ) with the round metric of constant curvature 4.

Proof. We prove the proposition when the quotient is $\mathbb{D}_{C}$, the another case follows in a similar fashion. In order to prove the statement, we consider the family $\mathfrak{G}$ of geodesics in $\mathbb{S}^{2 l-1}$ given by

$$
\mathfrak{G}:=\left\{\gamma(t)=\cos (t) x_{-}+\sin (t) x_{+}: P \in \mathbb{S}_{C}, x_{ \pm} \in E_{ \pm}^{1}(P)\right\}
$$

and we show that the following properties hold:

(1) Every geodesic in $\mathfrak{G}$ is orthogonal to the fibers of $\pi_{C}$ at all points.

(2) For every point $x \in \mathbb{S}^{2 l-1}$ and every vector $v$ normal to the fiber of $\pi_{C}$ through $x$, there is a geodesic in $\mathfrak{G}$ passing through $x$ and tangent to $v$.

(3) Every geodesic in $\mathfrak{G}$ projects to a unit speed geodesic in $\mathbb{D}_{C}$.

(1) It is enough to consider geodesics in $\mathfrak{G}$ with $t \in[0, \pi)$. There are two types of normal spaces to the fiber depending on the image of a point in the geodesic under $\pi_{C}$. If $\gamma(t)$ projects to an interior point of $\mathbb{D}_{C}$, that normal space is spanned by the vectors $X_{P_{i}}(\gamma(t))=P_{i} \gamma(t)-\left\langle P_{i} \gamma(t), \gamma(t)\right\rangle \gamma(t)$, as it was explained in Proposition 2.2 (3). On the contrary, if $\gamma(t)$ projects to $P \in \mathbb{S}_{C}$, the fiber through $\gamma(t)$ is $E_{+}^{1}(P)$ and its normal space is just $E_{-}(P)$. For $t=0$, the fiber through $\gamma(t)$ is $E_{-}^{1}(P)$ and the vector $\gamma^{\prime}(t)$ belongs to $E_{+}^{1}(P)$, so the geodesic is orthogonal to the fiber. Similarly for $t=\frac{\pi}{2}$, where the fiber is $E_{+}^{1}(P)$ and $\gamma^{\prime}(t)$ lies in $E_{-}^{1}(P)$. Otherwise, we have

$$
P \gamma(t)=\cos (t) P x_{-}+\sin (t) P x_{+}=-\cos (t) x_{-}+\sin (t) x_{+},
$$


and

$$
\langle P \gamma(t), \gamma(t)\rangle=-\cos ^{2}(t)+\sin ^{2}(t)=-\cos (2 t),
$$

which inserted into the formula for $X_{P}(x)$ results

$$
\begin{aligned}
X_{P}(\gamma(t))= & P \gamma(t)-\langle P \gamma(t), \gamma(t)\rangle \gamma(t) \\
= & -\cos (t) x_{-}+\sin (t) x_{+}+\cos (2 t)\left(\cos (t) x_{-}+\sin (t) x_{+}\right) \\
= & -\cos (t) x_{-}+\sin (t) x_{+}+\cos ^{3}(t) x_{-}+\cos ^{2}(t) \sin (t) x_{+} \\
& -\sin ^{2}(t) \cos (t) x_{-}-\sin ^{3}(t) x_{+} \\
= & -\cos (t) x_{-}+\sin (t) x_{+}+\left(1-\sin ^{2}(t)\right) \cos (t) x_{-} \\
& +\cos ^{2}(t) \sin (t) x_{+}-\sin ^{2}(t) \cos (t) x_{-}-\left(1-\cos ^{2}(t)\right) \sin (t) x_{+} \\
= & -2 \sin ^{2}(t) \cos (t) x_{-}+2 \sin (t) x_{+} \cos ^{2}(t) \\
= & \sin (2 t)\left(-\sin (t) x_{-}+\cos (t) x_{+}\right) \\
= & \sin (2 t) \gamma^{\prime}(t) .
\end{aligned}
$$

Hence $\gamma^{\prime}(t)=\frac{1}{\sin (2 t)} X_{P}(\gamma(t))$, which means it belongs to the normal space spanned by the $X_{P_{i}}(\gamma(t))$ basis.

(2) When $x \in \mathbb{S}^{2 l-1}$ projects to $P \in \mathbb{S}_{C}$, it belongs to the positive eigenspace $E_{+}^{1}(P)$ and, if $v$ is perpendicular to the fiber through $x$, then it belongs to $E_{-}(P)$. Therefore $\gamma(t)=$ $\cos (t) x+\sin (t) v$ belongs to $\mathfrak{G}$ and it satisfies $\gamma(0)=x, \gamma^{\prime}(0)=v$. When $x$ projects to a point in the interior of $\mathbb{D}_{C}$, any vector $v$ orthogonal to the fiber through $x$ is of the form $v=X_{P}(x)$ for some $P \in \mathbb{S}_{C}$. Such a $P$ gives a splitting $\mathbb{R}^{2 l}=E_{-}(P) \oplus E_{+}(P)$, and $x$ can be written as $\cos \left(t_{0}\right) x_{-}+\sin \left(t_{0}\right) x_{+}$for some $x_{ \pm} \in E_{ \pm}(P)$. Equation (2.8) means that $v$ is parallel to $\gamma^{\prime}\left(t_{0}\right)$, where $\gamma(t)=\cos (t) x_{-}+\sin (t) x_{+} \in \mathfrak{G}$. It follows from part (1) and (2) that the fibers of $\pi_{C}$ build a transnormal system on $\mathbb{S}^{2 l-1}$.

(3) Let us see first the form of a geodesic in $\mathbb{D}_{C}$ with the round metric of constant curvature 4 . Since $\mathbb{D}_{C}$ can be seen as the upper half part of a sphere of radius $\frac{1}{2}$, a geodesic with initial point $P \in \mathbb{D}_{C}$ has the general form $\gamma_{v}(t)=\cos (t) P+\sin (t) v$, for a vector $v \in \mathbb{D}_{C}$ and $v \perp P$. In particular, we can take $v:=Q \in \mathbb{D}_{C}$ such that $\langle P, Q\rangle=0$. Due to the homogenity of the geodesic equation, the unit speed geodesics in $\mathbb{D}_{C}$ are of the form

$$
\gamma_{\frac{Q}{\|Q\|}}(t)=\gamma_{Q}\left(\frac{t}{\|Q\|}\right)=\cos \left(\frac{t}{\|Q\|}\right) P+\sin \left(\frac{t}{\|Q\|}\right) Q=\cos (2 t) P+\sin (2 t) Q .
$$

Now, let us project to $\mathbb{D}_{C}$ a geodesic $\gamma(t)=\cos (t) x_{-}+\sin (t) x_{+}$in $\mathfrak{G}$, with $x_{ \pm} \in$ $E_{ \pm}^{1}\left(P_{0}\right), P_{0} \in \mathbb{S}_{C}$, where $P_{0}$ belongs to an orthonormal basis $\left(P_{0}, \ldots, P_{m}\right)$ for $\mathbb{R}_{C}$ 
(remember our computations do not depend on the choice of that basis). Then

$$
\begin{aligned}
\pi_{C}(\gamma(t)) & =\left\langle P_{0} \gamma(t), \gamma(t)\right\rangle P_{0}+\sum_{i=1}^{m}\left\langle P_{i} \gamma(t), \gamma(t)\right\rangle P_{i} \\
& =-\cos (2 t) P_{0}+2 \cos (t) \sin (t) \sum_{i=1}^{m}\left\langle P_{i} x_{+}, x_{-}\right\rangle P_{i} \\
& =\cos (2 t) P+\sin (2 t) Q
\end{aligned}
$$

where we have defined $P:=-P_{0}$ and $Q:=\sum_{i=1}^{m}\left\langle P_{i} x_{+}, x_{-}\right\rangle P_{i}$ in $\mathbb{D}_{C}$. Note that by definition $\langle-P, Q\rangle=0$. We deduce that the map $\pi_{C}$ sends unit speed geodesics on $\mathbb{S}^{2 l-1}$ to unit speed geodesics on the disk $\mathbb{D}_{C}$ with the round metric and hence the quotient Riemannian metric is that of constant curvature 4 .

Definition 2.7. Given a Clifford system $C$ of rank $m+1$ on $\mathbb{R}^{2 l}$ with $l \neq m+1$, we define the Clifford foliation $\mathcal{F}_{C}$ to be the foliation on $\mathbb{S}^{2 l-1}$ given by the fibers of $\pi_{C}$.

Remark 2.8. Note that Proposition 2.6 implies that any Clifford foliation $\mathcal{F}$ is a transnormal system.

Example 2.9 $(l=m)$. From Table 1.1, the value of $m$ can just be $1,2,4,8$. Let us discuss the case for $m=2$. By Proposition $2.2(2)$, the map is $\pi_{C}: \mathbb{S}^{3} \rightarrow \mathbb{S}^{2} \subseteq \mathbb{R}^{3}$. We need to find a Clifford system of rank 3 on $\mathbb{R}^{4}$. This implies a Clifford algebra representation $C_{1}=\mathbb{C}=\operatorname{span}\left\{1, e_{1}=i\right\}$ on $\mathbb{R}^{2}$. This refers us to Example 1.25 where we computed that representation. Now, for $x=(u, v) \in \mathbb{S}^{3} \subseteq \mathbb{C} \oplus \mathbb{C} \cong \mathbb{R}^{2} \oplus \mathbb{R}^{2}$,

$$
\begin{aligned}
\pi_{C}(x) & =\pi_{C}((u, v))=\left(\left\langle P_{0} x, x\right\rangle,\left\langle P_{1} x, x\right\rangle,\left\langle P_{2} x, x\right\rangle\right) \\
& =\left(u_{1}^{2}+u_{2}^{2}-v_{1}^{2}-v_{2}^{2}, 2 u_{1} v_{1}+2 u_{2} v_{2},-2 u_{1} v_{2}+2 u_{2} v_{1}\right) \\
& =\left(\|u\|^{2}-\|v\|^{2}, 2 \mathfrak{R e}(u \cdot \bar{v}), 2 \mathfrak{I m}(u \cdot \bar{v})\right)
\end{aligned}
$$

where "." denotes complex multiplication. Observe that they are the coordinates of the Hopf map $\mathbb{S}^{3} \rightarrow \mathbb{S}^{2}$. Make the circle act on $\mathbb{S}^{3}$ by the isometric group action on the right, discussed in Example 1.8. More precisely, for $z:=e^{i \theta} \in \mathbb{S}^{1} \subseteq \mathbb{C}$, the action $\mu: \mathbb{S}^{1} \rightarrow \mathbb{S}^{3}$, has the form

$$
\mu\left(e^{i \theta},(u, v)\right)=e^{i \theta} \cdot(u, v)=\left(e^{i \theta} u, e^{i \theta} v\right)
$$

Note that the Hopf map in eq. (2.10) is invariant under $\mathbb{S}^{1}$, i.e., $\pi_{C}(x)=\pi_{C}\left(e^{i \theta} \cdot x\right)$ since

$$
\begin{aligned}
\pi_{C}\left(e^{i \theta} \cdot x\right) & =\left(\left\|e^{i \theta}\right\|^{2}\left(\|u\|^{2}-\|v\|^{2}\right), 2 \mathfrak{R e}\left(e^{i \theta} u \cdot e^{-i \theta} \bar{v}\right), 2 \mathfrak{I m}\left(e^{i \theta} u \cdot e^{-i \theta} \bar{v}\right)\right) \\
& =\left(\|u\|^{2}-\|v\|^{2}, 2 \mathfrak{R e}(u \cdot \bar{v}), 2 \mathfrak{I m}(u \cdot \bar{v})\right)
\end{aligned}
$$

We just found that the Hopf fibration $\mathbb{S}^{1} \hookrightarrow \mathbb{S}^{3} \hookrightarrow \mathbb{S}^{2}$ is a Clifford foliation of $\mathbb{S}^{3}$, where each leaf (fiber) is a unit circle $\mathbb{S}^{1}$ and the leaf space is $\mathbb{S}^{2}$. The next beautiful images, taken from [Joh], show points in $\mathbb{S}^{2}$ with their corresponding preimages $\mathbb{S}^{1}$ through $\pi_{C}$. 

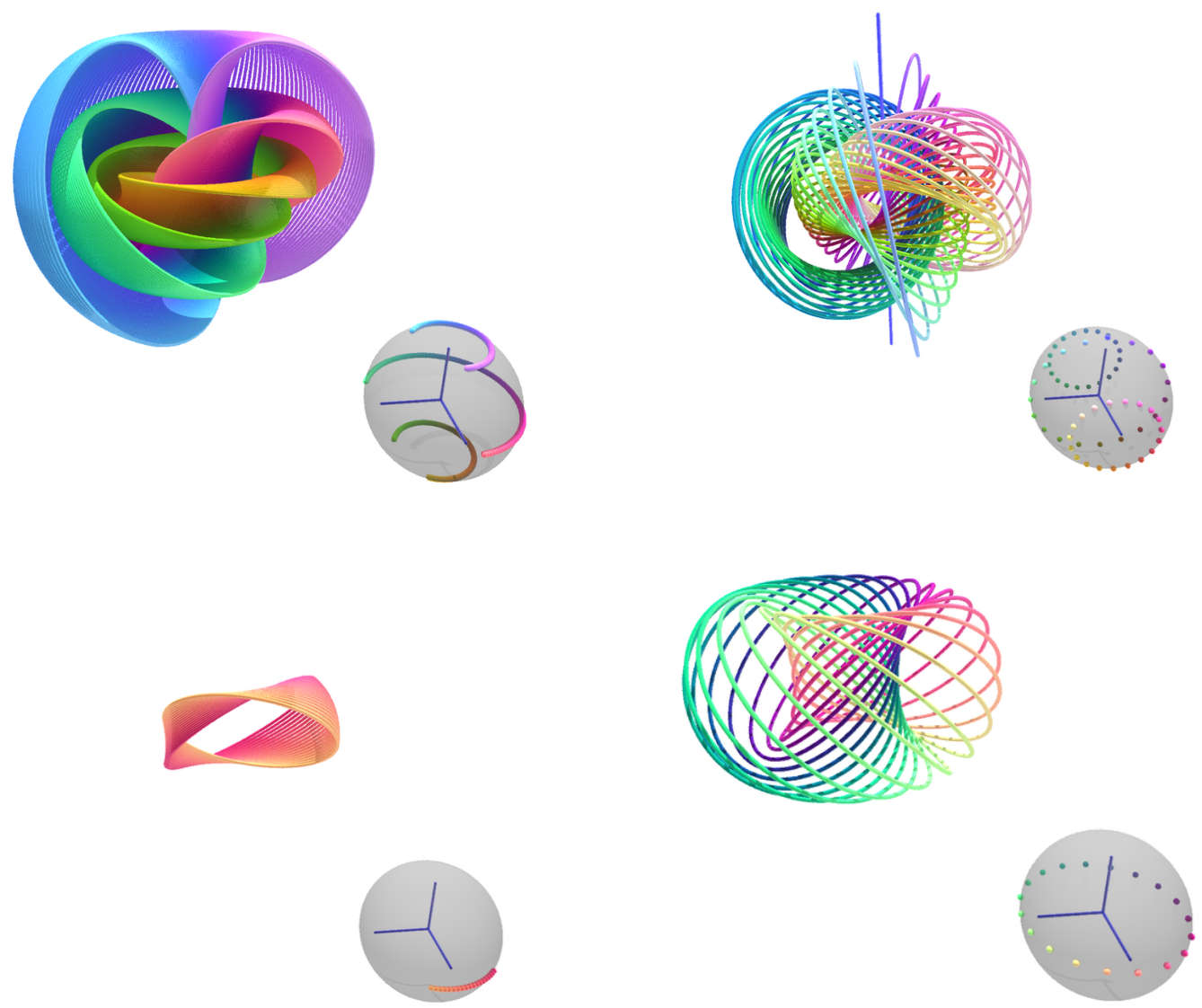

Figure 2.1: The Clifford foliation $\mathbb{S}^{3}$ induced from a Clifford system of rank 3 on $\mathbb{R}^{4}$.

Corollary 2.10. If $C$ is a Clifford system of rank $m+1$ on $\mathbb{R}^{2 l}$ and $l=m, \pi_{C}: \mathbb{S}^{2 m-1} \longrightarrow$ $\mathbb{S}_{C}$ is a Hopf fibration.

Proof. First and second parts in Proposition 2.2 shows that $\pi_{C}$ is a submersion with connected fibers of positive dimension for $l=m$, and in addition it is Riemannian by Proposition 2.6. The hypotheses in [Wil01, Theorem 1] (see also $\left[\mathrm{GG}^{+} 88\right.$, Corollary 5.4]) are satisfied and thus we conclude that $\pi_{C}$ is a Hopf fibration. The map is $\pi_{C}: \mathbb{S}^{2 m-1} \longrightarrow \mathbb{S}^{m}$, for $m=1,2,4,8$ as given in Table 1.1.

We finish this section showing that the FKM isoparametric hypersurfaces constructed in [FKM81] can be derived form Clifford foliations.

Corollary 2.11. When the image of $\pi_{C}$ is $\mathbb{D}_{C}$, i.e., $l>m+1$, with the round (also called hemisphere) metric, the preimages of the concentric spheres in $\mathbb{D}_{C}$ produce the FKM family of isoparametric hypersurfaces associated to $C$ in the sphere $\mathbb{S}^{2 l-1}$.

Proof. From Section 1.3 the Ferus, Karcher and Münzner polynomial in the sphere, $F_{0}$ : $\mathbb{S}^{2 l-1} \rightarrow[-1,1]$, given by

$$
F_{0}(x)=1-2 \sum_{i=0}^{m}\left\langle P_{i} x, x\right\rangle^{2} .
$$


For the map

$$
\begin{aligned}
f: \mathbb{D}_{C} & \longrightarrow[-1,1] \\
P & \longmapsto f(P)=1-2\|P\|^{2},
\end{aligned}
$$

we have $F_{0}=f \circ \pi_{C}$. Equipping $\mathbb{D}_{C}$ with the hemisphere metric, and the foliation $\mathcal{F}_{S}$ of concentric spheres, its quotient space $\Delta=\mathbb{D}_{C} / \mathcal{F}_{S}$ is the closed interval $\left[0, \frac{\pi}{4}\right]$. This induces a bijection between $[-1,1]$ and the leaf space $\left[0, \frac{\pi}{4}\right]$, where the focal manifold $M_{+}$ is represented by 1 in the first interval and by 0 (the origin in $\mathbb{D}_{C}$ ); likewise, $M_{-}$is -1 and $\frac{\pi}{4}$ (the boundary sphere $\mathbb{S}_{C}$ ). Thus, we recovered the FKM family whose leaves are given by the $\pi_{C}$-preimages $\sin (2 t) Q, Q \in \mathbb{S}_{C}$ in eq. (2.5) or, equivalently, for $x \in \mathbb{S}^{2 l-1}$ such that $\pi_{C}(x)=\sin (2 t) Q, t \in\left[0, \frac{\pi}{4}\right]$, and using the polynomial in eq. (2.11), then

$$
F_{0}(x)=1-2(\sin (2 t)\|Q\|)^{2}=1-2 \sin ^{2}(2 t)=\cos (4 t),
$$

as we described in Example 1.34.

\subsection{Composed foliations}

So far we have constructed Clifford foliations that have the property of being a transnormal system. In this section we will prove that in fact they are singular Riemannian foliations employing a method due to Lytchak [Lyt14] for a larger class of foliations on spheres that include Clifford foliations. This procedure involves the extension by homotheties of a singular Riemannian foliation $\mathcal{F}_{0}$ given on $\mathbb{S}_{C}$ to a foliation $\mathcal{F}_{0}^{h}$ of the same type on $\mathbb{D}_{C}$, and then sending the leaves of the latter back to $\mathbb{S}^{2 l-1}$ under the map $\pi_{C}$. The resulting foliation on $\mathbb{S}^{2 l-1}$ will be denoted by the composition $\mathcal{F}_{0} \circ \mathcal{F}_{C}$. Under this reasoning, the Clifford foliations we previously constructed in this chapter are singular Riemannian foliations when $\mathcal{F}_{0}$ is the finest foliation.

Lets start with setting up the composed foliation $\mathcal{F}_{0} \circ \mathcal{F}_{C}$ which turns out to be a transnormal system. For this aim, we first equip the disk $\mathbb{D}_{C}$ with a foliation inherited from the one on $\left(\mathbb{S}_{C}, \mathcal{F}_{0}\right)$. Fixing a Clifford system $C$ of rank $m+1$ on $\mathbb{R}^{2 l}$, the leaf space of the transnormal system $\left(\mathbb{S}^{2 l-1}, \mathcal{F}_{C}\right)$ is isometric to $\mathbb{S}_{C}$ or $\mathbb{D}_{C}$, both equipped with a metric of constant curvature 4 , due to Proposition 2.2. With such hemisphere metric, $\mathbb{D}_{C}$ can be described as a spherical join of $\mathbb{S}_{C}$ and a point (the origin) $\{p t\}$, which in this case is defined as the disjoint union of these two space together with every segment connecting any point from $\mathbb{S}_{C}$ to $\{p t\}$, and equipped with a metric of constant curvature 4 . From now on we will denote this space by $\frac{1}{2}\left(\mathbb{S}_{C} \star\{p t\}\right)$, where the factor $\frac{1}{2}$ represents a rescaling of the spherical join $\mathbb{S}_{C} \star\{p t\}$ with metric given by

$$
d^{2}(x, y)=t^{2}+s^{2}-2 t s \cos \left(d\left(x^{\prime}, y^{\prime}\right)\right)
$$

where $t, s$ are the respective distances from the origin to $x, y$, and $x^{\prime}, y^{\prime}$ the points in $\mathbb{S}_{C}$ lying in the rays passing through the origin and $x, y$ respectively (for further reference see 
[BBI01, p. 91]).

Take a closed transnormal system $\left(\frac{1}{2} \mathbb{S}_{C}, \mathcal{F}_{0}\right)$ on $\frac{1}{2} \mathbb{S}_{C}$, with leaf space $\frac{1}{2} \Delta$ and projection $\pi_{0}: \frac{1}{2} \mathbb{S}_{C} \longrightarrow \frac{1}{2} \Delta$. This leaf space is not necessarily a Riemannian manifold, thus we can not refer to this map as a Riemannian submersion. Nevertheless, we can still think on a similar concept for the context of metric spaces on which $\frac{1}{2} \Delta$ is included.

Definition 2.12. A map $f:\left(M, d_{M}\right) \rightarrow\left(N, d_{N}\right)$ between two metric spaces is a submetry if $f$ sends closed balls around a point to closed balls of the same radius around the image point. More precisely, if for any point $p \in M$ and radius $r$, the equality $f\left(B_{r}(p)\right)=B_{r}(f(p))$ holds.

It turns out that every Riemannian submersion is a submetry (see Propositions A.3 and A.4 and corollary A.5). From this fact we can conclude that, for $l=m, \pi_{C}$ is a submetry. In addition, Since the leaves of $\left(\frac{1}{2} \mathbb{S}_{C}, \mathcal{F}_{0}\right)$ are assumed to be closed, Corollary A.6 yields the projection $\pi_{0}$ is a submetry. Combining these two results, the composition $\pi_{0} \circ \pi_{C}$ gives a submetry $\mathbb{S}^{2 l-1} \rightarrow \frac{1}{2} \Delta$, see Proposition A.1. In the case for $l>m+1, \pi_{0}: \frac{1}{2} \mathbb{S}_{C} \longrightarrow \frac{1}{2} \Delta$ induces a third submetry

$$
\hat{\pi}_{0}: \frac{1}{2}\left(\mathbb{S}_{C} \star\{p t\}\right) \longrightarrow \frac{1}{2}(\Delta \star\{p t\}) .
$$

Composing $\hat{\pi}_{0}$ with $\pi_{C}: \mathbb{S}^{2 l-1} \longrightarrow \frac{1}{2}\left(\mathbb{S}_{C} \star\{p t\}\right)$, we obtain another submetry

$$
\hat{\pi}_{0} \circ \pi_{C}: \mathbb{S}^{2 l-1} \longrightarrow \frac{1}{2}(\Delta \star\{p t\})
$$

In either case we obtain a submetry $\mathbb{S}^{2 l-1} \rightarrow \underline{\Delta}$, where $\underline{\Delta}=\frac{1}{2} \Delta$ or $\frac{1}{2}(\Delta \star\{p t\})$, and its fibers are by construction the leaves of a transnormal system on $\mathbb{S}^{2 l-1}$, which we denote by $\mathcal{F}_{0} \circ \mathcal{F}_{C}$.

An equivalent and useful manner of describing $\mathcal{F}_{0} \circ \mathcal{F}_{C}$ is introducing the terminology of singular Riemannian foliations as follows. If we extend by homotheties the SRF $\mathcal{F}_{0}$ of the boundary $\mathbb{S}_{C}$ to $\mathcal{F}_{0}^{h}$ of the hemisphere $\mathbb{D}_{C}$, defining the leaf $L_{\lambda P}^{h}$ through the point $\lambda P$ as

$$
L_{\lambda P}^{h}:=\lambda \cdot L_{P}, \quad \text { for } P \text { in } \mathbb{S}_{C} \text { and } \lambda \in[0,1]
$$

Then the foliation $\mathcal{F}_{0} \circ \mathcal{F}_{C}$ is given by the preimages of the leaves in $\mathcal{F}_{0}^{h}$ under $\pi_{C}: \mathbb{S}^{2 l-1} \longrightarrow$ $\mathbb{D}_{C}$.

Moreover, notice that $\mathcal{F}_{0}^{h}$ is a $\mathrm{SRF}$ of $\mathbb{D}_{C}$, the interior of $\mathbb{D}_{C}$, since it is possible to extend the smooth vector fields $\left\{X_{i}\right\}$ that span the tangent space of each leaf of $\mathcal{F}_{0}$ by homotheties to $\mathcal{F}_{0}^{h}$ of the unit disk $\mathbb{D}_{C}$ : for $0 \leqslant t<1$, the family $\left\{t X_{i}\right\}$ of smooth vector fields span the tangent to $\mathcal{F}_{0}^{h}$ and, from the theory we already mentioned in section 1.1, the singular Riemannian foliation $\mathcal{F}_{0}$ is preserved by homotheties fixing the origin.

We are ready to state the central result of this section, stated in Proposition 2.15. The proof is divided in the two propositions below. The first assures that $\mathcal{F}_{0} \circ \mathcal{F}_{C}$, when restricted to each manifold, $M_{-}$and $\mathbb{S}^{2 l-1} \backslash M_{-}$, is a SRF. In the second is left the most 
technical part, that is the behavior of $\mathcal{F}_{0} \circ \mathcal{F}_{C}$ in a neighborhood "joining both pieces". More precisely, it is shown that $\mathcal{F}_{0} \circ \mathcal{F}_{C}$ is a SRF as well within a small enough neighborhood of a point belonging to $M_{-}$.

Proposition 2.13. If $\left(\mathbb{S}^{2 l-1}, \mathcal{F}_{C}\right)$ is a Clifford foliation and $\left(\mathbb{S}_{C}, \mathcal{F}_{0}\right)$ is a singular Riemannian foliation. The restrictions of $\mathcal{F}_{0} \circ \mathcal{F}_{C}$ to $M_{-}$and $\mathbb{S}^{2 l-1} \backslash M_{-}$are singular Riemannian foliations as well.

Proof. The statement is only proved when the quotient is $\mathbb{D}_{C}$, because it contains the case for $\mathbb{S}_{C}$. Applying Proposition $2.2(3)$, if the submetry from $\mathbb{S}^{2 l-1}$ to the hemisphere $\mathbb{D}_{C}$ is restricted to

$$
\left.\pi_{C}\right|_{\mathbb{S}^{2 l-1} \backslash M_{-}}: \mathbb{S}^{2 l-1} \backslash M_{-} \longrightarrow \stackrel{\circ}{\mathbb{D}}_{C},
$$

we obtain a Riemannian submersion, and since $\left(\mathbb{D}_{C}, \mathcal{F}_{0}^{h}\right)$ is a singular Riemannian foliation, we have that $\mathcal{F}_{0} \circ \mathcal{F}_{C}$ is again a SRF in $\mathbb{S}^{2 l-1} \backslash M_{-}$, due to Example 1.7. Similarly, since $\left(\mathbb{S}_{C}, \mathcal{F}_{0}\right)$ is a singular Riemannian foliation and $\left.\pi_{C}\right|_{M_{-}}: M_{-} \rightarrow \mathbb{S}_{C}$ is a Riemannian submersion by Proposition $2.2(1)$, the restriction $\left(M_{-},\left.\left(\mathcal{F}_{0} \circ \mathcal{F}_{C}\right)\right|_{M_{-}}\right)$is again a singular Riemannian foliation.

As we mentioned above, the goal in the next proposition is proving that for every point $x \in M_{-}$, there exists a neighborhood of $x \in \mathbb{S}^{2 l-1}$ in which the restriction of the foliation $\mathcal{F}_{0} \circ \mathcal{F}_{C}=: \mathcal{F}$ is a SRF.

Proposition 2.14. Consider a neighborhood $U \subseteq M_{-}$of a point $x \in M_{-}$, small enough that $\left.\nu\left(M_{-}\right)\right|_{U}$ admits an orthonormal frame $\left\{\xi_{1}, \ldots, \xi_{r}\right\}, r=\operatorname{codim}\left(M_{-}\right)$. Then the trivialization

$$
\begin{aligned}
\rho: U \times \mathbb{D}_{\varepsilon}^{r} & \longrightarrow \operatorname{Tub}_{\varepsilon}(U) \\
\left(x,\left(a_{1}, \ldots, a_{r}\right)\right) & \longmapsto \exp _{x}\left(\sum_{i} a_{i} \xi_{i}(x)\right)
\end{aligned}
$$

is a diffeomorphism, and $\rho^{*}\left(\left.\mathcal{F}\right|_{T u b_{\varepsilon}(U)}\right)=\left.\mathcal{F}\right|_{U} \times \mathcal{F}_{\mathbb{D}^{r}}$, where $\left(\mathbb{D}^{r}(\varepsilon), \mathcal{F}_{\mathbb{D}^{r}}\right)$ is the foliation of concentric spheres around the origin. In particular, $\left.\mathcal{F}\right|_{T u b_{\varepsilon}(U)}$ is a singular foliation around $M_{-}$.

Proof. By the definition of a distinguished tubular neighborhood (Definition 1.19), the existence of $U$ is clear since it could be a plaque in $L_{x}$ for $x$ restricted to $M_{-}$. Furthermore,

$$
T u b_{\varepsilon}(U)=\exp ^{\perp}\left(\nu^{\varepsilon}(U)\right), \quad \nu^{\varepsilon}(U)=\left\{v \in \nu(U) \mid v=\sum_{i=1}^{r} a_{1} \xi_{1}(x),\left\|\left(a_{1}, \ldots, a_{r}\right)\right\| \leqslant \varepsilon\right\}
$$

where, for a small enough $\varepsilon$, the normal exponential map is a diffeomorphism. That description is equivalent to the given by the map $\rho$ in the statement, then it is a diffeomorphism as well. In order to prove that $\rho$ induces a bijection between the leaf spaces, we lean on two maps as follows. On one hand, consider $U \times[0, \varepsilon]$, together with the foliation $\left.\mathcal{F}\right|_{U} \times\{p t s\}$. 
The map

$$
\begin{aligned}
(i d, r):\left(U \times \mathbb{D}^{r}(\varepsilon),\left.\mathcal{F}\right|_{U} \times \mathcal{F}_{\mathbb{D}^{r}}\right) & \longrightarrow\left(U \times[0, \varepsilon],\left.\mathcal{F}\right|_{U} \times\{p t s\}\right) \\
(u, v) & \longmapsto(u,\|v\|),
\end{aligned}
$$

where the concentric sphere of radius $r$ is sent to $r$ in $[0, \varepsilon]$, induces a bijection between leaf spaces. On the other hand, define

$$
\begin{aligned}
\left(\operatorname{Pr}, d_{U}\right):\left(T u b_{\varepsilon}(U), \mathcal{F}\right) & \longrightarrow\left(U \times[0, \varepsilon],\left.\mathcal{F}\right|_{U} \times\{p t s\}\right) \\
x & \longmapsto(\operatorname{Pr}(x), \operatorname{dist}(x, U)),
\end{aligned}
$$

where $\operatorname{Pr}$ is the metric projection for the tubular neighborhood and $\operatorname{dist}(\cdot, U)$ is the distance to $U$. Let us identify the leaf $L_{P}$ of $\left(\mathbb{S}_{C}, \mathcal{F}_{0}\right)$ with the class $[P] \in U /\left.\mathcal{F}\right|_{U}$. Using the description of the fibers of $\pi_{C}$ in eq. (2.5), the map $\left(\operatorname{Pr}, d_{U}\right)$ is taking the leaf of $\mathcal{F}$,

$$
M_{([P], t)}=\left\{\cos (t) x+\sin (t) Q x \mid x \in M_{+}, Q \in \pi_{C}\left(L_{P}\right)\right\},
$$

to the leaf $L_{P} \times\left\{\frac{\pi}{4}-t\right\}$. Since every leaf of $\mathcal{F}$ in $T u b_{\varepsilon}(U)$ is uniquely determined by $[P] \in U /\left.\mathcal{F}\right|_{U}$ and $t \in\left[\frac{\pi}{4}-\varepsilon, \frac{\pi}{4}\right]$, it follows that $\left(\operatorname{Pr}, d_{U}\right)$ induces a bijection between the leaf spaces as well. To conclude, note that if we compose $\left(\operatorname{Pr}, d_{U}\right)$ and $\rho$ we get the map $(i d, r)$,

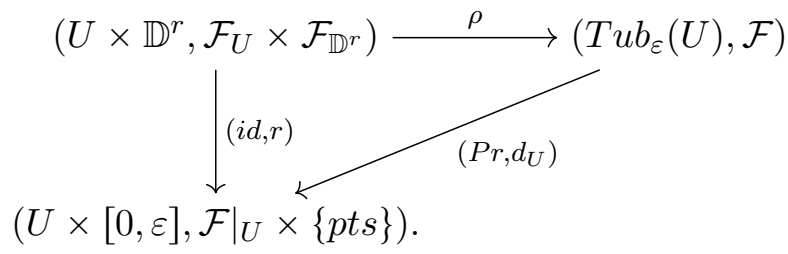

In detail,

$$
\begin{aligned}
\left(\left(\operatorname{Pr}, d_{U}\right) \circ \rho\right)\left(u,\left(a_{1}, \ldots, a_{r}\right)\right) & =\left(\operatorname{Pr}, d_{U}\right)\left(\sum_{i} a_{i} \xi_{i}(u)\right) \\
& =\left(\operatorname{Pr}\left(\sum_{i} a_{i} \xi_{i}(u)\right), \operatorname{dist}\left(\sum_{i} a_{i} \xi_{i}(u), U\right)\right) \\
& =\left(u,\left\|\left(a_{1}, \ldots, a_{r}\right)\right\|\right)=(i d, r),
\end{aligned}
$$

which in particular implies that $\rho$ induces a bijection between leaf spaces as well, and this finishes the proof.

By simply putting together the two latest propositions we conclude that $\left(\mathbb{S}^{2 l-1}, \mathcal{F}_{0} \circ \mathcal{F}_{C}\right)$ is a singular Riemannian foliation, as we summarize below.

Proposition 2.15. If $\left(\mathbb{S}^{2 l-1}, \mathcal{F}_{C}\right)$ is a Clifford foliation and $\left(\mathbb{S}_{C}, \mathcal{F}_{0}\right)$ is a singular Riemannian foliation, then $\mathcal{F}_{0} \circ \mathcal{F}_{C}$ is a singular Riemannian foliation as well.

Corollary 2.16. If $\mathcal{F}_{0}$ is a trivial foliation whose leaves consist of points, then $\mathcal{F}_{0} \circ \mathcal{F}_{C}=\mathcal{F}_{C}$ and, in particular, $\mathcal{F}_{C}$ is a singular Riemannian foliation. 
Proof. If $\mathcal{F}_{0}$ is the finest foliation in $\mathbb{S}_{C}$, then extending by homotopy $\mathcal{F}_{0}^{h}$ is the finest one as well, which implies $\mathcal{F}_{0} \circ \mathcal{F}_{C}=\mathcal{F}_{C}$ (since it is given by the fibers of $\pi_{C}$ ), and consequently $\mathcal{F}_{C}$ is a singular Riemannian foliation by Proposition 2.15.

Remark 2.17. Although the definition of a singular Riemannian foliation imposes the leaves to be connected, when $C$ is a Clifford system of rank $m+1$ on $\mathbb{R}^{2 l}$ and $l=m+1$, the foliation given by the non connected fibers of $\pi_{C}$ is a singular Riemannian foliation with disconnected fibers as established in [AR15, Section 3].

\subsection{Bijection between Clifford systems and singular Rieman- nian foliations}

We are going to prove in this section that there is a bijection between geometric equivalence classes of Clifford systems and congruence classes of singular Riemannian foliations in spheres whose quotient is a sphere or a hemisphere of curvature 4. In relation with previous results in this subject, the most general examples until the release of the Radeschi's paper were the FKM familes. It occurs not only that there exist geometric equivalence classes of Clifford systems giving rise the same isoparametric foliation, but also some isoparametric foliations not coming from Clifford systems. Hence the correspondence between Clifford systems and isoparametric foliations is far from being bijective. This also turns even more relevant the work in [Rad14]. The proof is divided into the next two propositions.

Proposition 2.18. Suppose $\left(\mathbb{S}^{n}, \mathcal{F}\right)$ is a singular Riemannian foliation such that the quotient space is a hemisphere $\frac{1}{2} \mathbb{D}^{m+1}$ of constant curvature 4 . Then $\mathcal{F}=\mathcal{F}_{C}$ for some Clifford system $C$.

Proof. Consider the boundary $\frac{1}{2} \mathbb{S}^{m}$ of $\frac{1}{2} \mathbb{D}^{m+1}$. Take an orthonormal basis of $\frac{1}{2} \mathbb{S}^{m}$, i.e., $m+1$ points $p_{0}, \ldots, p_{m}$ in that sphere mutually at distance $\frac{\pi}{4}$. Given a point $p_{i}$, take the partition of the $m+1$ disk $\frac{1}{2} \mathbb{D}^{m+1}$ into the distance spheres around $p_{i}$ and $-p_{i}$, which of course have codimension one, meaning that curves orthogonal to those spheres only have a direction to pass through. Since this is the behavior in the quotient space $\mathbb{D}^{m+1}$, when that partition is lifted via $\pi: \mathbb{S}^{n} \rightarrow \frac{1}{2} \mathbb{D}^{m+1}$, it induces a codimension 1 foliation $\mathcal{F}^{*}$ of $\mathbb{S}^{n}$ whose quotient is an interval of length $\frac{\pi}{2}$. Its singular leaves correspond to the two focal points $\pm p_{i}$. By the classification of such foliations ([Car38, Car39, Mün80, Mün81], alternatively see [CR15, Section 3.8]), the number of distinct principal curvatures is $g=2$, and the singular submanifolds are totally geodesic subspheres of $\mathbb{S}^{n}$,

$$
M_{0}=\{(x, y) \mid y=0\}=\mathbb{S}^{m_{1}} \times\{0\}, \quad M_{\frac{\pi}{2}}=\{(x, y \mid x=0)\}=\{0\} \times \mathbb{S}^{m_{2}},
$$

where $m_{1}+m_{2}=n-1$, and $m_{1}$ and $m_{2}$ correspond to the multiplicities of the curvatures in the Cartan-Münzner differential equations (Theorem 1.42). Besides, those subspheres lie on the same stratum, since they are preimage of points belonging to $\frac{1}{2} \mathbb{S}^{m}$; hence both singular leaves have the same dimension, $m_{1}=m_{2}=: l$. 
Now we are going to construct a Clifford system. Since $n=2 l+1, \mathbb{R}^{n+1}=\mathbb{R}^{2(l+1)}$ splits orthogonally as $V_{+}\left(p_{i}\right) \oplus V_{-}\left(p_{i}\right)$, where $V_{ \pm}\left(p_{i}\right)$ is the space spanned by the great sphere $\pi^{-1}\left( \pm p_{i}\right)$. Define a linear map $P_{i} \in \operatorname{Sym}_{2 l}(\mathbb{R})$ by

$$
\left.P_{i}\right|_{V_{+}\left(p_{i}\right)}=i d,\left.\quad P_{i}\right|_{V_{-}\left(p_{i}\right)}=-i d .
$$

From this we conclude that $P_{i}^{2}=i d$ and $E_{ \pm}\left(P_{i}\right)=V_{ \pm}\left(p_{i}\right)$. Doing the same for each $i$, we produce matrices $P_{0}, \ldots, P_{m}$ belonging to $\operatorname{Sym}_{2 l}(\mathbb{R})$, which are candidates for a basis in the Clifford system. Notice the proof finishes if we find that these maps anticommute or, equivalently, $P_{i}\left(E_{ \pm}\left(P_{j}\right)\right)=E_{\mp}\left(P_{j}\right)$ for $i \neq j$. It is enough to show that $P_{0}\left(E_{ \pm}\left(P_{1}\right)\right)=$ $E_{\mp}\left(P_{1}\right)$.

Let $x \in E_{+}\left(P_{0}\right)$ be a point in the preimage of $p_{0}$, and take a horizontal geodesic $\gamma$ starting at $x$ and tangent to the singular stratum such that $\pi(\gamma)$ passes through $p_{1}$. Since $\pi(\gamma)\left(\frac{\pi}{2}\right)=$ $-p_{0}$, the point $y=\gamma\left(\frac{\pi}{2}\right)$ belongs to $E_{-}\left(P_{0}\right)$ and we can write $\gamma(t)=\cos (t) x+\sin (t) y$. Furthermore, $z=\gamma\left(\frac{\pi}{4}\right)=\frac{\sqrt{2}}{2} x+\frac{\sqrt{2}}{2} y$ belongs to $E_{+}\left(P_{1}\right)$ by choice of $\gamma$. Then

$$
P_{0}(z)=P_{0}\left(\frac{\sqrt{2}}{2} x+\frac{\sqrt{2}}{2} y\right)=\frac{\sqrt{2}}{2} x-\frac{\sqrt{2}}{2} y=\gamma\left(-\frac{\pi}{4}\right) .
$$

But $\pi(\gamma)\left(-\frac{\pi}{4}\right)=-p_{1}$, which means $P_{0}(z) \in E_{-}\left(P_{1}\right)$.

Since any $z \in E_{+}^{1}\left(P_{1}\right)$ can be written as $\gamma\left(\frac{\pi}{4}\right)$ for some horizontal geodesic $\gamma$ from $E_{+}^{1}\left(P_{0}\right)$ to $E_{-}^{1}\left(P_{0}\right)$, we obtain that $P_{0}\left(E_{+}\left(P_{1}\right)\right) \subseteq E_{-}\left(P_{1}\right)$. Given that $P_{0}$ is nonsingular, by dimensional reasons it must be $P_{0}\left(E_{+}\left(P_{0}\right)\right)=E_{-}\left(P_{0}\right)$.

Proposition 2.19. If $C$ and $C^{\prime}$ are two different (geometric) equivalence classes of Clifford systems on $\mathbb{R}^{2 l}$ and $\mathbb{R}^{2 l^{\prime}}$ respectively, then there does not exist a foliated isometry between $\left(\mathbb{S}^{2 l-1}, \mathcal{F}_{C}\right)$ and $\left(\mathbb{S}^{2 l^{\prime}-1}, \mathcal{F}_{C^{\prime}}\right)$. In other words, Clifford foliations distinguish between (geometric) equivalence classes of Clifford systems.

Proof. Take $\left(P_{0}, \ldots, P_{m}\right)$ an orthonormal basis for $\mathbb{R}_{C}$ and $\left(Q_{0}, \ldots, Q_{m^{\prime}}\right)$ the corresponding one for $\mathbb{R}_{C^{\prime}}$. Unless $m=m^{\prime}$, the leaf spaces $\mathcal{F}_{C}$ and $\mathcal{F}_{C^{\prime}}$ have different dimensions, thus it cannot exist an isometry between them. If $m=m^{\prime}$, we have $l=k \delta(m)$ and $l^{\prime}=k^{\prime} \delta\left(m^{\prime}\right)=k^{\prime} \delta(m)$, which implies again that $\mathcal{F}_{C} \neq \mathcal{F}_{C^{\prime}}$, except for $k=k^{\prime}$. In that case we have two possible situations as we mentioned in Section 1.2: when $m \neq \equiv 0(\bmod 4)$ there exists a unique geometric class of Clifford systems for each $k$, whence $\mathcal{F}_{C} \cong \mathcal{F}_{C^{\prime}}$. Otherwise, $m \equiv 0(\bmod 4)$, the geometric class of $C$ is uniquely determined by the nonnegative integer $\left|\operatorname{tr}\left(P_{0}, \ldots, P_{m}\right)\right|$. Consequently, the proof is completed if we show that $\mathcal{F}_{C}$ and $\mathcal{F}_{C^{\prime}}$ are not congruent, unless $\left|\operatorname{tr}\left(P_{0}, \ldots, P_{m}\right)\right|=\left|\operatorname{tr}\left(Q_{0}, \ldots, Q_{m}\right)\right|$. This was already established in [FKM81, p. 486], as they showed that the invariant $\left|\operatorname{tr}\left(P_{0}, \ldots, P_{m}\right)\right|$ represents a characteristic number of the vector bundle

$$
\begin{aligned}
\Gamma:=\left\{(x, P) \in \mathbb{R}^{2 l} \times \mathbb{S}_{C} \mid P \in \mathbb{S}_{C}, x \in E_{+}(P)\right\} & \longrightarrow \mathbb{S}_{C} \\
(x, P) & \longmapsto P,
\end{aligned}
$$

whose sphere bundle is $\left.\pi_{C}\right|_{M_{-}}: M_{-} \longrightarrow \mathbb{S}_{C}$, where $M_{-}=\left\{(x, P) \in \mathbb{S}^{2 l-1} \times \mathbb{S}_{C} \mid P x=x\right\}$ 
We now want to discuss some recent works that have appeared after [Rad14], that have been contributing to a beautiful characterization of Clifford foliations through algebraic objects. We draw the reader's attention to this exciting connection between algebra and geometry which seems to be becoming stronger with the newest results about singular Riemannian foliations. The first one is by Lytchak and Radeschi in 2015 [LR15], where they proved that every singular Riemannian foliation with closed leaves can be described by polynomial equations. Then, its leaves are real algebraic subvarieties of the Euclidean space. Roughly speaking, they are a set of solutions of a system of polynomial equations over the real or complex numbers. And the second one is from 2016, in which Mendes and Radeschi [MR16] characterized closed singular Riemannian foliations of a Euclidean space, where the origin is a leaf (this is the infinitesimal model for SRF), as coming from quadratic homogeneous polynomials. This provides not only a strong and productive relationship between geometry and algebra in SRF's theory, but also opens the possibility of applying algebraic techniques to develop this branch of differential geometry. That paper also provides an algebraic characterization of Clifford foliations as those inhomogeneous infinitesimal foliations generated by homogeneous polynomials of degree two. 


\section{Chapter 3}

\section{Homogeneity in Clifford, FKM and composed foliations}

In this chapter we study the Clifford and composed foliations that are homogeneous. We start discussing some symmetry properties of Clifford foliations, then introducing some group actions concerning Clifford systems and finally we proof several homogeneity theorems relating FKM, Clifford and composed foliations.

\subsection{Relation between Clifford systems and some Lie groups}

Let $C$ a Clifford system of rank $m+1$ in $\mathbb{R}^{2 l}$. Examining the action of the elements belonging to the Clifford sphere in $\mathbb{S}_{C}$ over $\mathbb{S}^{2 l-1}$, we find symmetries of the Clifford foliations $\left(\mathbb{S}^{2 l-1}, \mathcal{F}_{C}\right)$ that can be described by some subgroups of the orthogonal group $\mathrm{O}(2 l)$.

Let $P \in \mathbb{S}_{C}$. By eq. (2.1) of $\pi_{C}$ we have that

$$
\left\langle\pi_{C}(P x), Q\right\rangle=\langle Q P x, P x\rangle, \quad \forall Q \in \mathbb{S}_{C} .
$$

By eq. (1.6), the equation above becomes

$$
\begin{aligned}
\left\langle\pi_{C}(P x), Q\right\rangle & =-\langle P Q x, P x\rangle+2\langle P, Q\rangle\langle I x, P x\rangle \\
& =-\langle Q x, x\rangle+2\langle P, Q\rangle\langle P x, x\rangle \\
& =-\left\langle\pi_{C}(x), Q\right\rangle+2\langle P, Q\rangle\left\langle\pi_{C}(x), P\right\rangle \\
& =\left\langle-\pi_{C}(x)+2\left\langle\pi_{C}(x), P\right\rangle P, Q\right\rangle,
\end{aligned}
$$

thus $\pi_{C}(P x)=-\pi_{C}(x)+2\left\langle\pi_{C}(x), P\right\rangle P$, which means $P$ sends the leaf of $\pi_{C}(x)$ in $\mathbb{S}^{2 l-1}$ to the one corresponding to the reflection along the segment through $P$ in $\mathbb{D}_{C}$, denoted by 
the map $\rho_{P}$. We can express this as a commutative diagram

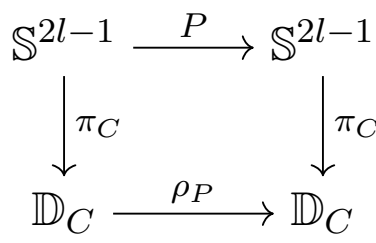

As we noted in section 1.2, the elements of the Clifford sphere in $\mathbb{S}_{C}$ are orthogonal matrices, so they belong to the group of orthogonal matrices of dimension $2 l$. Even more, since the composition of two reflections is a rotation, this is suggesting that an element $P Q$ lying in $\mathbb{S}_{C}$ produces a rotation. In fact, the elements in the Clifford sphere by definition generate the subgroup $\operatorname{Pin}(m+1) \subseteq \mathrm{O}(2 l)$, namely

$$
\operatorname{Pin}(m+1):=\left\langle P \mid P \in \mathbb{S}_{C}\right\rangle,
$$

and in turn, the Pin group contains the subgroup generated by an even number of products $P Q$,

$$
\operatorname{Spin}(m+1):=\left\langle P Q \mid P, Q \in \mathbb{S}_{C}\right\rangle .
$$

Furthermore, since every element $P$ in $\mathbb{S}_{C}$ is a orthogonal linear transformation, then automatically it is an isometry, and moreover it can be thought as a foliated isometry of $\left(\mathbb{S}^{2 l-1}, \mathcal{F}_{C}\right)$. Equation (3.1) shows that $\operatorname{Spin}(m+1)$ acts over each point $\mathbb{D}_{C}$ reflecting $\pi_{C}(x)$ along the segment through $P \in \operatorname{Spin}(m+1)$. Since $\operatorname{Spin}(m+1)$ is connected, we have a covering $\eta: \operatorname{Spin}(m+1) \rightarrow \mathrm{SO}(m+1)$ whose induced action on $\mathbb{D}_{C}$ has (by the Cartan-Dieudonné theorem) all the concentric spheres around the origin as the principal orbits - which means this Spin action is isometric and has cohomogeneity 1. In particular, when $x$ lies in $M_{+}, \pi_{C}(P x)=0$, whence, $\pi_{C}\left(M_{+}\right)$is the only singular orbit. The quotient $\mathbb{D}_{C} / \operatorname{Spin}(m+1)$ is then isometric to $\left[0, \frac{\pi}{4}\right]=\mathbb{S}^{2 l-1} / \mathcal{F}_{C}^{\prime}$, where is the FKM foliation corresponding to the Clifford system $C$.

The Pin group seen as a subgroup of the orthogonal matrices is defined by the reflection about the hyperplane orthogonal to an element in the Pin group. Nevertheless, that geometric insight adds an inconvenient negative sign in the treatment of Pin and Spin groups. In order to handle this problem we will use the twisted adjoint representation, which requires the introduction of both an automorphism and an anti-homomorphism in a Clifford algebra over a finite-dimensional vector space (for a reference see [LM89, Chapter 1]).

Definition 3.1. Every Clifford algebra $C_{m}$ has a unique canonical automorphism $\alpha: C_{m} \rightarrow$ $C_{m}$ defined by

$$
A \rightarrow \alpha(A)= \begin{cases}1, & \text { if } A=1 \\ -e_{i}, & \text { if } A=e_{i} \\ (-1)^{k} e_{i_{1}} \cdots e_{i_{k}}, & \text { if } A=e_{i_{1}} \cdots e_{i_{k}} \text { and } 1 \leqslant e_{i_{1}}<\cdots<e_{i_{k}} \leqslant m .\end{cases}
$$


Definition 3.2. We define conjugation on a Clifford algebra $C_{m}$ as the anti-automorphism

$$
A \rightarrow \bar{A}= \begin{cases}1, & \text { if } A=1 \\ -e_{i}, & \text { if } A=e_{i} \\ (-1)^{k} e_{i_{k}} \cdots e_{i_{1}}, & \text { if } A=e_{i_{1}} \cdots e_{i_{k}} \text { and } 1 \leqslant e_{i_{1}}<\cdots<e_{i_{k}} \leqslant m .\end{cases}
$$

Now we have the enough machinery to describe a surjective homomorphism from the Pin group to the orthogonal group.

Definition 3.3. We define the twisted adjoint representation on a Clifford system as the map

$$
\begin{aligned}
\mathcal{R}: \operatorname{Pin}(m+1) & \longrightarrow \mathrm{O}(m+1) \\
P_{i} & \longmapsto \mathcal{R}_{P_{i}}(x)=\alpha\left(P_{i}\right) x \overline{P_{i}},
\end{aligned}
$$

where it is enough defining $\mathcal{R}$ in the elements $P_{i}$ of an orthonormal basis $\left(P_{0}, \ldots, P_{m}\right)$ for $\mathbb{R}_{C}$ and $x$ in $\mathbb{D}_{C}$, since we are working over a finite-dimensional vector space.

The next proposition shows how the twisted adjoint representation is just the algebraic characterization of the simple geometric concept of reflection in a hyperplane. Even further, this reveals that Clifford algebras generalize quaternions, which was the original idea of creating those objects. See [Gal12, Proposition 1.6] for a proof.

Proposition 3.4. For every nonzero element $x \in \mathbb{D}_{C}$, the twisted adjoint representation $\mathcal{R}_{P_{i}}$ is the reflection about the hyperplane orthogonal to the vector $P_{i}$. That is,

$$
\mathcal{R}_{P_{i}}(x)=x-2\left\langle P_{i}, x\right\rangle P_{i}
$$

Notice the Pin group double covers the orthogonal group by means of $\mathcal{R}_{P_{i}}$ since $\mathcal{R}_{P_{i}}(x)=\mathcal{R}_{-P_{i}}(x)$, for all $x$ in $\mathbb{D}_{C}$. Due to the same construction, it turns out the Spin group is the double cover of the special orthogonal group, as well. We sum up all discussed before in the following diagram:

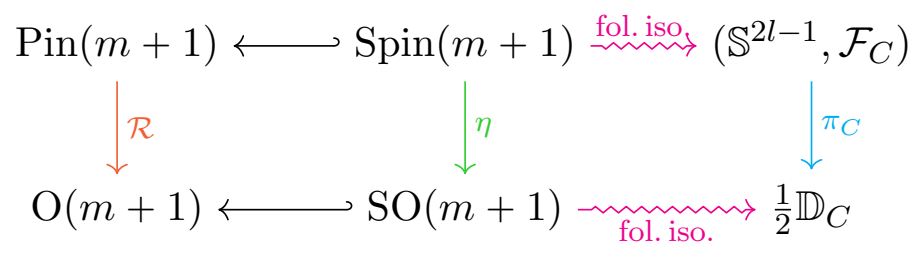

\subsection{Homogeneity in Clifford, FKM and composed foliations}

In this section we study the Clifford and composed foliations that are homogeneous. In the case when the image of $\pi_{C}$ is the boundary $\mathbb{S}_{C}$, there are just two possibilities happening in dimensions $m=2$ or 4 . Otherwise, the analysis is more extensive and it results very interesting homogeneity relations among the Clifford $\mathcal{F}_{C}$, the FKM $\mathcal{F}_{C}^{\prime}$, the singular Riemannian $\mathcal{F}_{0}$ and the composed $\mathcal{F}_{0} \circ \mathcal{F}_{C}$ foliations. 
From item (2) in Proposition 2.2 we know the image of $\pi_{C}$ is $\mathbb{S}_{C}$ when $l=m$ and $m$ just can be $1,2,4$ or 8 , according to Table 1.1. For $m=1$, the only possible singular Riemannian foliations are the coarsest or the finest ones. However, neither of them are given by the preimages of $\pi_{C}$, so they are not of Clifford type. For $m=2,4$ and 8 , we showed in Corollary 2.10 they are all Hopf fibrations. Nevertheless, in the latter case, $m=8$, the works of Guijarro and Walshap [GW07], Lytchak and Wilking [LW16, Wil01] and Gromoll and Grove $\left[\mathrm{GG}^{+} 88\right]$ proved that $\pi_{C}: \mathbb{S}^{15} \rightarrow \mathbb{S}^{8}$ is the only non-homogeneous regular foliation, given by the fibers of a Riemannian submersion. Then, it turns out that the only homogeneous cases are when $m$ is 2 (see Example 2.9) or 4 (see again $\left[\mathrm{GG}^{+} 88\right.$, LW16]).

Once exhausted the possibilities for $\mathbb{S}_{C}$, hereafter we assume the quotient $\mathbb{S}^{2 l-1} / \mathcal{F}_{C}$ is the hemisphere $\mathbb{D}_{C}$, unless otherwise stated. We begin comparing homogeneity between the Clifford foliation $\mathcal{F}_{C}$ and the FKM foliation $\mathcal{F}_{C}^{\prime}$ on $\mathbb{S}^{2 l-1}$.

Proposition 3.5. Let $C$ a Clifford system of rank $m+1$ on $\mathbb{R}^{2 l}$ such that $l>m+1$. If $\mathcal{F}_{C}$ is homogeneous, then $\mathcal{F}_{C}^{\prime}$ is homogeneous as well.

Proof. Suppose that $\left(\mathbb{S}^{2 l-1}, \mathcal{F}_{C}\right)$ is given by the action of a Lie subgroup $H \subseteq \mathrm{SO}(2 l)$. Let $G \subset \mathrm{SO}(2 l)$ be the topological closure of the group generated by $H$ and the image of the spin representation $\eta: \operatorname{Spin}(m+1) \rightarrow \mathrm{SO}(m+1)$ we defined in section 3.1. Since both $H$ and $\operatorname{Spin}(m+1)$ act by foliated isometries on $\left(\mathbb{S}^{2 l-1}, \mathcal{F}_{C}\right)$, so does $\mathrm{G}$. As we analyzed before, the spin action already generates all the action of $\mathrm{SO}(m+1)$ on $\mathbb{D}_{C}$; besides, $H$ gives the identity action on $\mathbb{D}_{C}$. Then, the $G$-action also has cohomogeneity 1 on $\mathbb{D}_{C}$. In particular, the orbits of $G$ in $\mathbb{S}^{2 l-1}$ are the leaves generated by the FKM family and thus $\mathcal{F}_{C}^{\prime}$ is homogeneous.

In [FKM81, GWZ $\left.{ }^{+} 08\right]$ are found the conditions for a FKM foliation on $\mathbb{S}^{2 l-1}$ to be homogeneous. This result is summarized in Table 3.1, where are listed Clifford systems $C$ of rank $m+1$ on $\mathbb{R}^{2 l}, l=k \delta(m)$ and $l>m+1$. For instance, the condition $P_{0} P_{1} P_{2} P_{3} P_{4}= \pm I d$, which is the product of the Clifford matrices $P_{i}$ in $C$, appears in [FKM81, Section 5] to ensure that both focal manifolds are homogeneously embedded. From Proposition 3.5, those

\begin{tabular}{c||c|c|c|c}
$(m, k)$ & $(1, k)$ & $(2, k)$ & $(4, k)$ & $(9,1)$ \\
\hline Condition & $k \geqslant 2$ & $k \geqslant 1$ & $k \geqslant 1, P_{0} P_{1} P_{2} P_{3} P_{4}= \pm I d$ & -
\end{tabular}.

Table 3.1: Homogeneity for the FKM foliation on the sphere.

four cases are the only cases with a chance to give a homogeneous Clifford foliation on $\mathbb{S}^{2 l-1}$. We now are going to examine the first three situations, i.e., for $m=1,2$ and 4 . The proof is basically a version of [FKM81, Theorem 6.1] adapted to Clifford foliations.

Proposition 3.6. For a Clifford system $C$ of rank $m+1$ on $\mathbb{R}^{2 l}, l=k \delta(m)$ and $l>m+1$, it holds that:

- If $m=1, \mathcal{F}_{C}$ is given by the orbits of the diagonal action $\mathrm{SO}(k)$-action on $\mathbb{S}^{2 k-1} \subseteq$ $\mathbb{R}^{k} \oplus \mathbb{R}^{k}$ 
- If $m=2, \mathcal{F}_{C}$ is given by the orbits of the diagonal action $\mathrm{SU}(k)$-action on $\mathbb{S}^{4 k-1} \subseteq$ $\mathbb{C}^{k} \oplus \mathbb{C}^{k}$

- If $m=4$ and $P_{0} P_{1} P_{2} P_{3} P_{4}= \pm I d, \mathcal{F}_{C}$ is given by the orbits of the diagonal action $\operatorname{Sp}(k)$-action on $\mathbb{S}^{8 k-1} \subseteq \mathbb{H}^{k} \oplus \mathbb{H}^{k}$.

Proof. After using the construction for a Clifford system $C$ given in section 1.2, we are going to prove that every orbit generated by the group action is contained in a fiber of $\pi_{C}$ and vice versa. For all cases, $m=1,2$ or $m=4$ and $P_{0} P_{1} P_{2} P_{3} P_{4}= \pm I d, C$ can be set up as follows: Consider $\mathbb{F} \in\{\mathbb{R}, \mathbb{C}, \mathbb{H}\}$ the division algebra such that $\operatorname{dim}_{\mathbb{R}} \mathbb{F}=m$ and let $j_{1}, \ldots, j_{m-1}$ the canonical imaginary units of $\mathbb{F}$. For an element belonging to $\mathbb{F}$, $x=x_{0}+x_{1} j_{1}+\cdots+x_{m-1} j_{m-1}, x_{i} \in \mathbb{R}$, the real part of $x$ is $\mathfrak{R e}(x)=x_{0}$ and its $r$-th imaginary part is given by $\mathfrak{I m}_{r}(x)=x_{r}=\mathfrak{R e}\left(x \cdot-j_{r}\right)$, for $r=1, \ldots, m-1$. Define the Clifford system $C=\left(P_{0}, \ldots, P_{m}\right)$ on $\mathbb{R}^{2 k \delta(m)}=\mathbb{R}^{2 k m}=\mathbb{F}^{k} \times \mathbb{F}^{k}$,

$$
P_{0}(u, v)=(u,-v), \quad P_{1}(u, v)=(v, u), \quad P_{1+i}(u, v)=\left(j_{r} \cdot v,-j_{r} \cdot u\right), \quad 1 \leqslant r \leqslant m-1,
$$

where $u=\left(u_{1}, \ldots, u_{k}\right), v=\left(v_{1}, \ldots, v_{k}\right)$ are in $\mathbb{F}^{k}$.

Generalizing example 2.9, notice that the projection $\pi_{C}$ is determined by the functions

$$
\left\{\begin{array}{l}
\left\langle P_{0}(u, v),(u, v)\right\rangle=\|u\|^{2}-\|v\|^{2} \\
\left\langle P_{1}(u, v),(u, v)\right\rangle=2 \mathfrak{R e}(u \cdot \bar{v}) \\
\left\langle P_{r+1}(u, v),(u, v)\right\rangle=2 \mathfrak{R e}\left(u \cdot \bar{v} \cdot-j_{r}\right)=2 \mathfrak{I m}_{r}(u \cdot \bar{v}),
\end{array}\right.
$$

which is equivalently to write

$$
\pi_{C}(u, v)=\left(\|u\|^{2}-\|v\|^{2}, 2 u \cdot \bar{v}\right) \in \mathbb{R} \oplus \mathbb{F} .
$$

Let $\mathrm{U}(\mathbb{F}, k)$ to be a Lie group defined by $\mathrm{SO}(k), \mathrm{SU}(k), \mathrm{Sp}(k)$ according to whether the field $\mathbb{F}$ is $\mathbb{R}, \mathbb{C}, \mathbb{H}$ respectively. By constructing an orthonormal basis from Gram-Schmidt process and proving that the orbit of a single point is all the sphere, $\mathrm{U}(\mathbb{F}, k)$ yields a transitive action on $\mathbb{S}^{m k-1} \subseteq \mathbb{F}^{k}$. Thus its diagonal action also preserves the functions in eq. (3.3), since

$$
(A u, A v)=\left(\|u\| A \frac{u}{\|u\|},\|v\| A \frac{v}{\|v\|}\right)=(u, v),
$$

where $A \in \mathrm{U}(\mathbb{F}, k)$ and we applied transitivity in the third expression. In particular, the orbits of such action are contained in the fibers of $\pi_{C}$, and therefore in the leaves of $\mathcal{F}_{C}$. Moreover, any point $(u, v) \in \mathbb{S}^{m k-1} \subseteq \mathbb{F}^{k} \times \mathbb{F}^{k}$ can be moved by the $\mathrm{U}(\mathbb{F}, k)$-action to a point of the form

$$
\left(u_{1} e_{1}, v_{1} e_{1}+v_{2} e_{2}\right)
$$

where $e_{1}, e_{2}$ are the elements of the canonical basis on $\mathbb{F}^{k}$, and $v_{1} \in \mathbb{F}, u_{1}, v_{2} \in \mathbb{R}_{\geqslant 0}$ and $u_{1}^{2}+\left|v_{1}\right|^{2}+v_{2}^{2}=1$. Furthermore, there is only one point of the form in eq. (3.4) for each fiber of $\pi_{C}$, because the functions in eq. (3.3) determine $u_{1}, v_{1}, v_{2}$ uniquely. For if 
$\left(p_{1} e_{1}, q_{1} e_{1}+q_{2} e_{2}\right) \in \mathbb{S}^{2 m k-1}, p_{1}, q_{2} \in \mathbb{R}_{\geqslant 0}$ and $q_{1} \in \mathbb{F}$, is another element such that

$$
\left(2 p_{1}^{2}-1,2 p_{1} q_{1},-2 p_{1} q_{2}\right)=\left(2 u_{1}^{2}-1,2 u_{1} v_{1},-2 u_{1} v_{2}\right)=\pi_{C}\left(u_{1} e_{1}, v_{1} e_{1}+v_{2} e_{2}\right),
$$

then from the first component $u_{1}=p_{1}$, since both must be positive. This yields $v_{1}=q_{1}$ in the second component, and $v_{2}=q_{2}$ in the third one. In particular, every point in a fiber of $\pi_{C}$ can be moved to a specific point via the $\mathrm{U}(\mathbb{F}, k)$-action, hence the orbits of that group coincide with the leaves of $\mathcal{F}_{C}$.

The next result analyzes the last case in Table 3.1 and it gives an example where the contrary to Proposition 3.5 fails. Namely, for $m=9$, the Clifford foliation $\left(\mathbb{S}^{31}, \mathcal{F}_{C}\right)$, induced by the Clifford system $\left(P_{0}, \ldots, P_{9}\right)$ on $\mathbb{R}^{32}$, is inhomogeneous even though the FKM foliation $\left(\mathbb{S}^{31}, \mathcal{F}_{C^{\prime}}\right)$ is homogeneous. Our proof contains a slightly different argument from the one given by Radeschi in [Rad14, Proposition 5.3] that we consider it simplifies the reasoning. Specifically we used a result of Gorodski and Lytchak [GL16, Theorem 1] in order to prove that the quotient $\mathbb{S}^{n} / G_{0}$ cannot be $\frac{1}{2} \mathbb{S}^{10}$.

Proposition 3.7. The Clifford foliation $\left(\mathbb{S}^{31}, \mathcal{F}_{C}\right)$ induced by the Clifford system $\left(P_{0}, \ldots, P_{9}\right)$ of rank 10 on $\mathbb{R}^{32}$ is not homogeneous.

Proof. Suppose by contradiction that $\left(\mathbb{S}^{31}, \mathcal{F}_{C}\right)$ is homogeneous, i.e., the foliation is given by the orbits of Lie subgroup $G \subseteq \mathrm{SO}(32)$ acting by isometries. First, let us see that assumption implies that the principal isotropy group $H$ must be trivial. If it is not the case, then we consider the fix set of $H$, which must be an $n$-dimensional subsphere of $\mathbb{S}^{31}$,

$$
\operatorname{Fix}(H):=\left\{x \in \mathbb{S}^{31} \mid \forall h \in H, h \cdot x=x\right\}=\mathbb{S}^{n} .
$$

By definition of a principal isotropy group, $\mathbb{S}^{n}$ meets all the orbits, in particular it meets the principal orbits. Define $G^{\prime}:=N(H) / H$, where $N(H)$ is the normalizer of $H$ in $G$. We can interpret $G^{\prime}$ as the remaining group when the elements acting trivially are removed from the normalizer. From these two last observations, the identity component $G_{0}^{\prime}$ of $G^{\prime}$ acts on $\mathbb{S}^{n}$ with trivial principal isotropy groups, i.e., $G_{0}^{\prime}$ does not fix any element of $\mathbb{S}^{n}$. The quotient $\mathbb{S}^{31} / G$ is a Riemannian orbifold since it is isometric to $\frac{1}{2} \mathbb{D}_{C}$ and hence all the slice representations $\left(G_{p}, \nu_{p}(G(p))\right)$ are polar [LT10]. In order to simplify the study of that quotient, we are going to construct an orbifold covering - we refer the reader to a brief review in Riemannian orbifolds in appendix B. The Luna-Richardson-Straume reduction (see [GL16, Section 2.6]) of $G$ on $\mathbb{S}^{31}$ to the induced action of $N(H) / H$ on $\operatorname{Fix}(H)$, states that there is a canonical isometry

$$
\mathbb{S}^{n} / N(H) \longrightarrow \mathbb{S}^{31} / G
$$

where the action of $N(H)$ on $\mathbb{S}^{n}$ has $H$ in its kernel. Such reduction has trivial principal isotropy groups and the same quotient as the original space, that is,

$$
\mathbb{S}^{n} / G^{\prime}=\mathbb{S}^{31} / G
$$

Since the polarity condition depends only on the connected component, applying again the 
Lytchak and Thorbergsson result, $\mathbb{S}^{n} / G^{\prime}$ is orbifold if and only if $\mathbb{S}^{n} / G_{0}^{\prime}$ is orbifold, which in this situation holds from eq. (3.5).

Given that $G_{0}^{\prime} \subseteq G^{\prime} \subseteq G$, the local model for orbifold coverings implies that

$$
\mathbb{S}^{n} / G_{0}^{\prime} \longrightarrow \mathbb{S}^{31} / G
$$

is a Riemannian orbi-covering. The orbifold $\mathbb{S}^{31} / G=\frac{1}{2} \mathbb{D}_{C}$ has two (up to isomorphism) possible orbi-covers: $\frac{1}{2} \mathbb{S}^{10}$ or $\frac{1}{2} \mathbb{D}^{10}$ (see Example B.7). However, it cannot be the first case since $\mathbb{S}^{n} / G_{0}^{\prime}$ fails to be $\frac{1}{2} \mathbb{S}^{10}$, due to [GL16, Theorem 1]; whence, $\mathbb{S}^{n} / G_{0}^{\prime}=\frac{1}{2} \mathbb{D}^{10}$. Applying Proposition 2.18, the foliation on $\mathbb{S}^{n}$ given by the orbits of $G_{0}^{\prime}$ corresponds to a Clifford foliation system $C=\left(P_{0}, \ldots, P_{9}\right)$ on $\mathbb{R}^{2 l}$. Thus, $l=k \delta(m)$ and $l \geqslant \delta(9)=16$. This means that $n \geqslant 31$, i.e., $\mathbb{S}^{n}=\mathbb{S}^{31}$ and $H=\{1\}$, the principal isotropy group is trivial as we wanted proved.

From Proposition $2.2(1), E_{+}^{1}\left(P_{0}\right) \cong \mathbb{S}^{15}$ is a leaf of $\left(\mathbb{S}^{31}, \mathcal{F}_{C}\right)$. Then $G$ acts transitively on $\mathbb{S}^{15}$. Using the fact that $G$ has trivial principal isotropy groups, thus $\operatorname{dim} G=31-10=21$. However, there are no groups of dimension 21 acting transitively on $\mathbb{S}^{15}$ (see $\left[\mathrm{GWZ}^{+} 08\right.$, Table C]). Then, $\left(\mathbb{S}^{31}, \mathcal{F}_{C}\right)$ is not homogeneous.

The work of Gorodski and Lytchak [GL16] that we just used in the previous proof classifies representations of compact connected Lie groups $G$ acting on the unit sphere $\mathbb{S}^{n}$ for which orbit space $\mathbb{S}^{n} / G=: \mathcal{O}$ is isometric to a Riemannian orbifold. The main result is stated in Theorem 1, which contains necessary and sufficient conditions of the representation and the identity component of the action group for the quotient $\mathcal{O}$ being a Riemannian orbifold. As a first corollary of their main result, they prove that the universal covering $\tilde{\mathcal{O}}$ of the orbit space $\mathcal{O}$ is just one of the next four: a weighted complex, a weighted quaternionic projective space (which happens if and only if $\tilde{\mathcal{O}}$ has dimension 2 or the action is almost free and has rank 1 [Str94, $\mathrm{GG}^{+} 88$ ]; or it has constant curvature 1 (if and only if the action is polar [Dad85]) or 4. The second corollary says that the quotients are orbifolds of low dimension, $2 \leqslant \operatorname{dim} \tilde{\mathcal{O}} \leqslant 5$ (the case of dimension 2 was already classified by Straume in [Str94]), when $G$ has rank at least 2 and the representation is not polar. Comparing this with Radeschi work, notice the quotient space $\mathbb{D}_{C}^{m+1}$ provides orbifolds of arbitrary dimension, arising from a dimensionally large enough sphere $\mathbb{S}^{2 l-1}$. The quotients are hemispheres, quarter-spheres and eighth-spheres.

In the next proposition we determine the homogeneity of $\mathcal{F}_{0} \circ \mathcal{F}_{C}$ in terms of the homogeneity of the $\mathcal{F}_{0}, \mathcal{F}_{C}, \mathcal{F}_{C}^{\prime}$ foliations.

Proposition 3.8. Let $C, \mathcal{F}_{C}$ and $\mathcal{F}_{C}^{\prime}$ be as in Proposition 3.5, and let $\left(\mathbb{S}_{C}, \mathcal{F}_{0}\right)$ be a singular Riemannian foliation. If the leaf space of $\mathcal{F}_{C}$ is a hemisphere and the composed foliation $\mathcal{F}_{0} \circ \mathcal{F}_{C}$ is homogeneous, then $\mathcal{F}_{0}$ and $\mathcal{F}_{C}^{\prime}$ are homogeneous. On the other hand, if $\mathcal{F}_{0}$ and $\mathcal{F}_{C}$ are homogeneous, so is $\mathcal{F}_{0} \circ \mathcal{F}_{C}$.

Proof. Suppose first that $G$ is a group which induces the foliation $\left(\mathbb{S}^{2 l-1}, \mathcal{F}_{0} \circ \mathcal{F}_{C}\right)$. Take an arbitrary point $x$ in the leaf $M_{+}$of $\mathcal{F}_{0} \circ \mathcal{F}_{C}$. There always exists a $\varepsilon>0$ such that the 
normal exponential map at $x$ is a diffeomorphism on the normal sphere $\nu_{x}^{\varepsilon}\left(M_{+}\right)$of radius $\varepsilon$. Since $\exp _{x}^{\perp}$ is also a $G_{x}$-equivariant map (where $G_{x}$ is the isotropy group of $x$ ), then there is a bijective correspondence between orbits in $\nu_{x}^{\varepsilon}\left(M_{+}\right)$and the leaves in $\mathcal{F}_{0} \circ \mathcal{F}_{C}$, restricted to the tube of radius $\varepsilon$ around $x$. By definition of that composed foliation, we have, once again, a bijection between the leaves in $\mathcal{F}_{0} \circ \mathcal{F}_{C}$ restricted to the $\varepsilon$-tube and the ones in the sphere of radius $\varepsilon$ around 0 of $\mathcal{F}_{0}^{h}$. From all that, we have got until here that to each orbit in $\nu_{x}^{\varepsilon}\left(M_{+}\right)$corresponds a leaf $\mathcal{F}_{0}^{h}$ in $\mathbb{S}^{m}(\varepsilon)$ via the map $\pi_{C} \circ \exp ^{\perp}$, and viceversa. Applying the homothetic transformation used for the definition of $\mathcal{F}_{0}^{h}$, we can extend the latter bijection to the unit sphere $\mathbb{S}^{m}=\mathbb{S}_{C}$, and finally get that $\mathcal{F}_{0}$ is homogeneous under the identity component of the isotropy group $\left(G_{x}\right)^{0} \subseteq\left(G^{0}\right)_{x}$. It left proving $\mathcal{F}_{C}^{\prime}$ is homogeneous, which is easier since we can use the same idea as in Proposition 3.5. Consider a subgroup $G^{\prime} \subseteq \mathrm{SO}(2 l)$, generated by $G$ and the spin representation $\eta(\operatorname{Spin}(m+1))$. Therefore, the leaves of $\mathcal{F}_{C}^{\prime}$ corresponds to the $G^{\prime}$-orbits in $\mathbb{S}^{2 l-1}$, so it is homogeneous, too.

Suppose now that $\left(\mathbb{S}_{C}, \mathcal{F}_{0}\right)$ is homogeneous, given by the orbits of a representation $\rho: H \rightarrow \mathrm{SO}(m+1)$. Up to double cover $H^{\prime} \rightarrow H$ we can lift $\rho$ to $\rho^{\prime}: H^{\prime} \rightarrow \operatorname{Spin}(m+1)$, and via the embedding $\eta: \operatorname{Spin}(m+1) \rightarrow \mathrm{SO}(2 l)$ stated in Definition 3.3 we have a representation $\rho^{\prime \prime}: H^{\prime} \rightarrow \mathrm{SO}(2 l)$. The orbits induced from $\rho^{\prime \prime}\left(H^{\prime}\right)$ on $\mathbb{S}^{2 l-1}$ descend via $\pi_{C}$ to $\rho(H)$-orbits on $\mathbb{D}_{C}$, due to the properties of $\eta$. In addition, if $\mathcal{F}_{C}$ is generated by a group $K$, once again we apply the same argument used in Proposition 3.5 to define $K^{\prime}$ as the (topological) closure group formed by $K$ and $\rho^{\prime \prime}\left(H^{\prime}\right)$, and we get it acts on $\mathbb{S}^{2 l-1}$ by isometries and its orbits are the leaves of $\left(\mathbb{S}^{2 l-1}, \mathcal{F}_{0} \circ \mathcal{F}_{C}\right)$.

Corollary 3.9. If $\left(\mathbb{S}^{2 l-1}, \mathcal{F}_{C}\right)$ is a Clifford foliation with quotient $\mathbb{S}^{2}$ or $\mathbb{S}^{4}$, then for every singular Riemannian foliation $\left(\mathbb{S}_{C}, \mathcal{F}_{0}\right)$ the composed foliation $\mathcal{F}_{0} \circ \mathcal{F}_{C}$ is homogeneous.

Proof. Since the quotient is a sphere, we automatically know that $\mathcal{F}_{C}$ is homogeneous from the explanation at the beginning of Section 3.2. Besides, when $\mathcal{F}_{0}$ is a singular Riemannian foliation on $\mathbb{S}^{2}$ or $\mathbb{S}^{4}$, then $\mathcal{F}_{0}$ is the coarsest foliation in $\mathbb{S}^{4}$, in which case it is trivially homogeneous, or it has $\operatorname{dim} \mathcal{F}_{0} \leqslant 3$. For this second case, Radeschi showed that foliation is homogeneous (see $\left[\operatorname{Rad} 12\right.$, Main Theroem]). Then we have that both foliations $\mathcal{F}_{C}$ and $\mathcal{F}_{0}$ are homogeneous, then the composed $\mathcal{F}_{0} \circ \mathcal{F}_{C}$ is homogeneous by Proposition 3.8.

The next result uses all we studied in this section. The aim is to establish a necessary and sufficient condition (independently of the leaf space) between homogeneity in $\mathcal{F}_{0} \circ \mathcal{F}_{C}$ and $\mathcal{F}_{0}, \mathcal{F}_{C}$, except for two cases: foliations coming from Clifford systems of rank 9 and 10 on $\mathbb{R}^{2 l}$.

Proposition 3.10. Let $C$ be a Clifford system on $\mathbb{R}^{2 l}$ and $\left(\mathbb{S}_{C}, \mathcal{F}_{0}\right)$ a singular Riemannian foliation. If the Clifford system $C$ on $\mathbb{R}^{2 l}$ is different from rank 9 or 10 , then $\left(\mathbb{S}^{2 l-1}, \mathcal{F}_{0} \circ\right.$ $\left.\mathcal{F}_{C}\right)$ is homogeneous if and only if $\mathcal{F}_{0}$ and $\mathcal{F}_{C}$ are homogeneous. If $C$ has rank 10 , then $\left(\mathbb{S}^{2 l-1}, \mathcal{F}_{0} \circ \mathcal{F}_{C}\right)$ is homogeneous only if $\mathcal{F}_{0}$ is homogeneous.

Proof. From Corollary 3.9 and Proposition 3.8, $\mathcal{F}_{0} \circ \mathcal{F}_{C}$ is homogeneous whenever $\mathcal{F}_{0}$ and $\mathcal{F}_{C}$ are homogeneous. Notice we have excluded both $C$ of rank 9 and 10 since they are inhomogeneous, as we discussed at the beginning of this section and in Proposition 3.7. 
Assuming now that $\mathcal{F}_{0} \circ \mathcal{F}_{C}$ is homogeneous, we consider two situations, depending of the sort of quotient space given in $\mathbb{S}^{2 l-1}$ by the Clifford foliation:

- If the leaf space of $\mathcal{F}_{C}$ is $\mathbb{S}_{C}$, then Corollary 3.9 and Proposition 3.6 allows us to conclude that necessarily $\mathcal{F}_{C}$ must be homogeneous with $C$ of rank 3 or 5 . So we are done, since $\mathcal{F}_{0}$ is homogeneous in both cases as was proved in that result.

- If the leaf space of $\mathcal{F}_{C}$ is $\mathbb{D}_{C}$, thus $\mathcal{F}_{0}$ and $\mathcal{F}_{C}^{\prime}$ are homogeneous, due to Proposition 3.8. Then, $\mathcal{F}_{C}$ is homogeneous as well, except when $C$ is of rank 10 (by Table 3.1 and Proposition 3.6).

Some months after the publication of [Rad14], his author, in joint work with Gorodski [GR16], completed the classification of homogeneous, singular Riemannian foliations on $\mathbb{S}^{2 l-1}$ given by $\pi_{C}$. Specifically, they dealt with composed foliations $\mathcal{F}_{0} \circ \mathcal{F}_{C}$ produced by the Clifford systems of rank 9 and 10 on $\mathbb{R}^{16}$ and $\mathbb{R}^{32}$, respectively, those not considered in Proposition 3.10 above. In the former case, they found exactly 6 examples of homogeneous composed foliations of $\mathbb{S}^{15}$, listed in Tables 1 and 2 of that paper. In the latter, they proved there is only one homogeneous foliation in $\mathbb{S}^{31}$, which is isoparametric and it is induced by the action on $\operatorname{Spin}(10)$ via the spin representation. In this case, $\left(\mathbb{S}^{9}, \mathcal{F}_{0}\right)$ corresponds to the coarsest foliation.

Their main result also allowed to establish that there are some foliations, whose leaf space has constant curvature 4, which are not composed foliations. Namely, those given by the action of $\operatorname{Spin}(9)$ and $\operatorname{Spin}(9) \cdot \mathrm{SO}(2)$ on $\mathbb{R}^{31}$.

In [Rad14, Section 6.3], Radeschi proved that for any $\operatorname{SRF}\left(\mathbb{S}^{8}, \mathcal{F}_{0}\right)$ there corresponds a SRF $\tilde{\mathcal{F}}_{0}$ of the Cayley projective plane $\mathbb{O} P^{2}$, which is homogeneous if and only if the composed foliation $\left(\mathbb{S}^{15}, \mathcal{F}_{0} \circ \mathcal{F}_{C}\right)$, induced from a Clifford system $C$ of rank 10 , is homogeneous as well. Regarding this case, it is a consequence of the main theorem in [GR16] that all the singular Riemannian foliations in the Cayley projective plane $\left(\mathbb{O} P^{2}, \tilde{\mathcal{F}}_{0}\right)$, corresponding to a $\operatorname{SRF}\left(\mathbb{S}^{8}, \mathcal{F}_{0}\right)$, are inhomogeneous, except for those six examples. 
62 HOMOGENEITY IN CLIFFORD, FKM AND COMPOSED FOLIATIONS 


\section{Appendix A}

\section{Riemannian submersions and submetries}

There is some important facts that are commonly used in the literature of singular Riemannian foliations but it is not common finding the proofs in papers or textbooks. In this section proofs of some of these very known results that have been employed throughout this dissertation are written.

Proposition A.1. Let $f: X \rightarrow Y$ a submetry between metric spaces where $Y$ is connected. Then the following properties holds:

(a) $f$ is 1-Lipschitz.

(b) $f$ is open.

(c) $f$ is surjective.

(d) If $g: Y \rightarrow Z$ is another submetry to a metric space $Z$, then the composition $g \circ f$ is a submetry.

Proof. (a) Let $x_{1}, x_{2} \in X$ and define $r:=d_{X}\left(x_{1}, x_{2}\right)$. Since $f$ is a submetry and $x_{2}$ lies in the closed ball $B_{r}\left[x_{1}\right]$, we have that $f\left(B_{r}\left[x_{1}\right]\right)=B_{r}\left[f\left(x_{1}\right)\right] \ni f\left(x_{2}\right)$. That is equivalent to say $f$ is 1-Lipschitz because

$$
d_{Y}\left(f\left(x_{1}\right), f\left(x_{2}\right)\right) \leqslant r=d_{X}\left(x_{1}, x_{2}\right) .
$$

(b) Let $U$ be an open set in $X$ and take an $f(x) \in f(U)$, for $x \in U$. Since $U$ is open we can find $\varepsilon, \delta$ such that $0<\delta<\varepsilon$ and $B_{\delta}[x] \subseteq B_{\varepsilon}[x] \subseteq U$. Using in addition that $f$ is a submetry we can deduce that $f(U)$ is open since,

$$
B_{\delta}(f(x)) \subseteq B_{\delta}[f(x)]=f\left(B_{\delta}[x]\right) \subseteq f\left(B_{\varepsilon}[x]\right) \subseteq f(U)
$$

where $B_{\delta}(f(x))$ is an open ball lying on $Y$ and containing $f(x)$. Consequently, $f$ is an open map.

(c) It is enough showing that the image of $f$ is closed, since $Y$ is connected and we just proved in (b) that $f$ is an open map. Let $\left\{x_{n}\right\}_{n \in \mathbb{N}}$ a sequence in $X$ such that 
$\left\{f\left(x_{n}\right)\right\}_{n \in \mathbb{N}}$ is a sequence in $f(X)$ converging to an element $y \in Y$. This means that for every $\varepsilon$, there exists $n_{0} \in \mathbb{N}$ such that for all $n>n_{0}, d\left(f\left(x_{n}\right), y\right)<\varepsilon$. Now, $y \in B_{\varepsilon}\left[f\left(x_{n_{0}+1}\right)\right]=f\left(B_{\varepsilon}\left[x_{n_{0}+1}\right]\right)$ because $f$ is submetry. This implies $y=f\left(z_{n_{0}+1}\right)$, for some $z_{n_{0}+1} \in B_{\varepsilon}\left[x_{n_{0}+1}\right]$, whence, $y$ belongs to $f(X)$ and consequently the image of $f$ is closed.

(d) Due to submetry, we already know that $f\left(B_{r}[x]\right)=B_{r}[f(x)]$. Composing $g$ in both sides of the latter equality and using submetry again we find

$$
g \circ f\left(B_{r}[x]\right)=g\left(B_{r}[f(x)]\right)=B_{r}[g \circ f(x)] .
$$

Thus, the composition of submetries is a submetry.

Before proving the next propositions about submetries, we need to define the notion of an almost equidistant partition.

Definition A.2. Let $X$ a metric space. A partition $\bigcup_{i \in I} X_{i}$ of $X$ is called almost equidistant if the distance between any two elements $X_{i}$ and $X_{j}$ belonging to it is given by

$$
d\left(X_{i}, X_{j}\right)=d\left(x_{i}, X_{j}\right)=d\left(X_{i}, x_{j}\right)
$$

for all $x_{i}$ and $x_{j}$ lying in $X_{i}$ and $X_{j}$, respectively.

Note this definition is relevant since the infimum in $d\left(x_{i}, X_{j}\right):=\inf \left\{d\left(x_{i}, x_{j}\right) \mid x_{j} \in X_{j}\right\}$ might not be attained. If for each pair of elements $X_{i}$ and $X_{j}$ in the partition $\bigcup_{i \in I} X_{i}$ of $X$ such infimum is attained, the partition is called equidistant.

Proposition A.3. Let $f: X \rightarrow Y$ a submetry between metric spaces such that $X$ is proper. Then its fibers are almost equidistant. Moreover, if $\bigcup_{i \in I} X_{i}=X$ is an almost equidistant partition of a metric space $X$, then the projection $f: X \rightarrow Y$ to its quotient space is a submetry.

Proof. For the first assertion, let $y_{1}, y_{2} \in Y$ two different elements and define $X_{1}:=f^{-1}\left(y_{1}\right)$, $X_{2}:=f^{-1}\left(y_{2}\right)$. The fiber distance in $X$ is defined as

$$
d\left(X_{1}, X_{2}\right):=\inf \left\{d\left(x_{1}, X_{2}\right) \mid x_{1} \in X_{1}\right\}:=\inf \left\{d\left(x_{1}, x_{2}\right) \mid \text { for all } x_{1} \in X_{1}, x_{2} \in X_{2}\right\} .
$$

Call $r:=d\left(X_{1}, X_{2}\right), r>0$. Since $X$ is proper, the fibers of $f$ are closed and the infimum in the definition above is attained. This means there exist $x_{1} \in X_{1}$ and $x_{2} \in X_{2}$ such that $d\left(x_{1}, x_{2}\right)=r$, in other words, $x_{2}$ belongs to the closed ball $B_{r}\left[x_{1}\right]$, centered in $x_{1}$ and radius $r$. Since $f$ is a submetry we have that $y_{2}=f\left(x_{2}\right) \in f\left(B_{r}\left[x_{1}\right]\right)=B_{r}\left[y_{1}\right]$, for all $x_{1} \in X_{1}$. Now let $x_{2}^{\prime} \in X_{2}$. Due to submetry it holds that $f\left(B_{r}\left[x_{2}^{\prime}\right]\right)=B_{r}\left[y_{2}\right] \ni y_{1}$, whence, there exists $x_{1}^{\prime} \in B_{r}\left[x_{2}^{\prime}\right]$, such that $f\left(x_{1}^{\prime}\right)=y_{1}$. Then,

$$
d\left(X_{1}, X_{2}\right) \leqslant d\left(X_{1}, x_{2}^{\prime}\right) \leqslant d\left(x_{1}^{\prime}, x_{2}^{\prime}\right) \leqslant r=d\left(X_{1}, X_{2}\right)
$$

and consequently $d\left(x_{1}^{\prime}, x_{2}^{\prime}\right)=r$. Thus the fibers of $f$ are almost equidistant (in fact, they are equidistant since the fibers are closed). 
For the second affirmation, note that for $x_{0} \in X$ and $r \geqslant 0$, the inclusion $f\left(B_{r}\left[x_{0}\right]\right) \subseteq$ $B_{r}\left[f\left(x_{0}\right)\right]$ certainly holds since a projection is distance non-increasing. For the other inclusion, let $y \in B_{r}\left[f\left(x_{0}\right)\right]$ and consider the fibers of $y_{0}:=f\left(x_{0}\right)$ and $y$, which belong to the partition in $X$, as $X_{0}=f^{-1}\left(y_{0}\right)$ and $X_{1}:=f^{-1}(y)$, respectively. From the definition of distance in the quotient space we have that

$$
d\left(y_{0}, y\right)=d\left(X_{0}, X_{1}\right)=d\left(x_{0}, X_{1}\right):=\inf \left\{R>0 \mid B_{R}\left[x_{0}\right] \cap X_{1} \neq \varnothing\right\} \leqslant r
$$

where we fixed $x_{0}$ since the partition given in $X$ is almost equidistant. The definition of infimum implies that for all integer $n>0$ there exist a number $R_{n}>0$ such that $R_{n} \leqslant r+\frac{1}{n}$ and $B_{R_{n}}\left[x_{0}\right] \cap X_{1} \neq \varnothing$. Let $x_{n}$ an element in that intersection, then $d\left(x_{0}, x_{n}\right) \leqslant r+\frac{1}{n}$ and $f\left(x_{n}\right)=y$. Therefore, $f\left(x_{n}\right)=y \in f\left(B_{r}\left[x_{0}\right]\right)$, hence $B_{r}\left[f\left(x_{0}\right)\right] \subseteq f\left(B_{r}\left[x_{0}\right]\right)$, since $x_{n}$ converges when $n \rightarrow \infty$, and $f$ is a submetry.

Proposition A.4. Let $\tilde{M}, M$ be complete Riemannian manifolds and $\pi: \tilde{M} \rightarrow M$ a Riemannian submersion. Then its fibers form an equidistant partition.

Proof. Let $X_{1}$ and $X_{2}$ in $\tilde{M}$ two fibers over to $y_{1}$ and $y_{2}$ in $M$, respectively. From the Hopf-Rinow theorem, let $\gamma:[0,1] \rightarrow M$ be a minimizing geodesic between $y_{1}$ and $y_{2}$ in $M$. Since $\pi$ is a Riemannian submersion, we can take the horizontal lift $\tilde{\gamma}$ of $\gamma$, starting at an arbitrary point $x_{1}=\tilde{\gamma}(0)$ in the fiber of $y_{1}$. Since $\pi$ is distance non-increasing (see [GHL12, Section 2.C.6]), there is a $x_{2} \in X_{2}$ which is the endpoint of $\tilde{\gamma}$. Now, for $d\left(X_{1}, X_{2}\right)$ it holds that

$$
d\left(X_{1}, X_{2}\right) \leqslant d\left(x_{1}, x_{2}\right) \leqslant \mathcal{L}(\tilde{\gamma})=\mathcal{L}(\gamma)=d\left(y_{1}, y_{2}\right) .
$$

Furthermore, the distance between $X_{1}$ and $X_{2}$ is realized by a horizontal geodesic whose projection is a geodesic of the same length, then $d\left(y_{1}, y_{2}\right) \leqslant d\left(X_{1}, X_{2}\right)$. From those inequalities, we conclude that $d\left(X_{1}, X_{2}\right)=d\left(y_{1}, y_{2}\right)$ and we have proved that the fibers of $\pi$ are equidistant.

Corollary A.5. Every Riemannian submersion between complete Riemannian manifolds is a submetry.

Proof. The fibers of any Riemannian submersion form an equidistant partition, due to Proposition A.4. The result follows from Proposition A.3 since every Riemannian submersion is in particular a map between proper metric spaces.

A singular Riemannian foliation $(M, \mathcal{F})$ is called closed if all its leaves are closed. In such case, the transnormal system condition is equivalent to all the leaves being at a constant distance from each other. From Proposition A.3 we immediately obtain the following corollary:

Corollary A.6. Let $(M, \mathcal{F})$ be a closed singular Riemannian foliation and $\pi:(M, \mathcal{F}) \rightarrow \Delta$ the projection to its leaf space. Then $\pi$ is a submetry. 


\section{Appendix B}

\section{Riemannian Orbifolds}

In this appendix we introduce some basic definitions of orbifolds, specially the Riemannian ones, which can be pictured like a topological space with singularities, given locally by the not necessarily free action of a finite group of isometries, and also we talk about their coverings and fundamental groups. All this notions are explicitly used in Chapter 3, to prove some homogeneity relations among composed foliations $\mathcal{F}_{0} \circ \mathcal{F}_{C}, \mathcal{F}_{0}$ and $\mathcal{F}_{C}$ (see Propositions 3.7 and 3.10). For a wider exploration in Orbifolds we recommend the following sources: Thurston [Thu02], Gorodski [Gor14] and Davis [Dav10].

Definition B.1. A metric space $X$ is a Riemannian orbifold if every point $x \in X$ admits a neighborhood $U$ isometric to a quotient $M / \Gamma$, where $M$ is a Riemannian manifold and $\Gamma$ is a finite group of isometries.

Example B.2. The leaf space $\mathbb{S}^{2 l-1} / \mathcal{F}_{C}$ of a homogeneous Clifford foliation, such as the ones studied on chapter 3 , is barely a Riemannian manifold, but a metric space. In fact, It turns out this is a Riemannian orbifold, where the sphere $\left(\mathbb{S}^{2 l-1}, g_{\text {round }}\right)$ is equipped with the metric inherited from the Euclidean space and the action is given by isometries of a connected Lie group $G$, whose orbits form the foliation $\mathcal{F}_{C}$. For instance, the isometric induced action of $\eta: \operatorname{Spin}(m+1) \rightarrow \mathrm{SO}(m+1)$ on $\mathbb{D}_{C}$ whose quotient is isometric to $\left[0, \frac{\pi}{4}\right]=\mathbb{S}^{2 l-1} / \mathcal{F}_{C}^{\prime}$, where $\mathcal{F}_{C}^{\prime}$ is the FKM foliation corresponding to the Clifford system $C$ (see discussion in section 3.1).

In what follows, unless otherwise indicated, we will consider theory for Riemannian orbifolds since it is the context we are concerned. Reducing spaces to quotients is not the only way of making more comprehensible a geometrical object; it is also advantageous considering more general (often simpler) structures which give rise to those spaces we are interested in. In such direction, covering orbifold spaces and orbifold fundamental groups allow us analyzing orbifolds, by analogy with manifolds. Roughly speaking, while the local model for coverings over manifolds are homeomorphisms (isometries in Riemannian manifolds), an orbifold covering locally looks like a map $\mathbb{R}^{n} / \tilde{\Gamma} \rightarrow \mathbb{R}^{n} / \Gamma$, where $\tilde{\Gamma} \subseteq \Gamma$ are groups of isometries on $\mathbb{R}^{n}$.

Definition B.3. A covering orbifold or orbi-cover of an orbifold $\mathcal{O}$ is an orbifold $\tilde{\mathcal{O}}$ together with a projection $\pi: \tilde{\mathcal{O}} \rightarrow \mathcal{O}$ such that each point $x \in \mathcal{O}$ has a neighborhood $U$ isometric to $M / G$, where $M$ is a Riemannian manifold and $G$ a group of isometries, for which each 
connected component $U_{i}$ of $\pi^{-1}(U)$ is isometric to $M / G_{i}$ for some subgroup $G_{i} \subseteq G$ such that the isometries respect the projection.

It is well known (see, for example, [Thu02]) that every connected orbifold admits an universal orbi-covering $\tilde{\mathcal{O}}$, that is, a space which orbi-covers any other orbi-covering space of $\mathcal{O}$, and it is unique up to equivalence.

The following two definition works for orbifolds in general, not only Riemannian ones.

Definition B.4. The orbifold fundamental group $\pi_{1}^{\text {orb }}(\mathcal{O})$ of an orbifold $\mathcal{O}$ is the group of deck transformations of the universal cover $\tilde{\mathcal{O}}$ - homeomorphisms on $\tilde{\mathcal{O}}$ which not only permute the elements on each fiber, but also leave the projection invariant.

Definition B.5. An orbifold is simply connected if it is connected and does not admit a nontrivial orbi-cover.

Remark B.6. The universal orbi-covering is always simply connected, as in manifolds. However, notice that orbifolds can be simply-connected in the topological sense without being simply-connected in the orbifold sense. This indicates that the orbifold fundamental group is a refinement of the fundamental group for manifolds.

Example B.7. In order to link the last concepts to our study in chapter 3, let us calculate the fundamental group of the disk $\frac{1}{2} \mathbb{D}_{C}^{10}$ (appearing in the proof about non-homogeneity for the Clifford system of rank 10 on $\mathbb{R}^{32}$, proposition 3.7 ). Of course, $\frac{1}{2} \mathbb{D}_{C}^{10}$ trivially orbicovers itself. However, the orbifold $\frac{1}{2} \mathbb{S}^{10}$ not only orbi-covers that disk but also it is simply connected, then it is the universal covering (up to equivalence) for that hemisphere. Thus the group of deck transformations is $\pi_{1}^{\text {orb }}\left(\frac{1}{2} \mathbb{D}_{C}^{10}\right) \cong \mathbb{Z}_{2}$.

Example B.8. Let the cyclic group $\mathbb{Z}_{n}$ acting by rotations around a fixed axis on the sphere $\mathbb{S}^{2}$. The orbit of an element $x$ in the 2 -sphere corresponds to $n$ evenly spaced points in the level circle of $x$ around the fixed axis. The leaf space is a Riemannian orbifold that looks like an American football ball with a cusp on both extremes, which is topologically a 2 -sphere. Furthermore, the universal covering is $\mathbb{S}^{2}$, since it is simply connected and the identity map is trivially a homeomorphism, whence, its fundamental group is $\pi_{1}^{\text {orb }}\left(\mathbb{S}^{2}\right) \cong \mathbb{Z}_{n}$. 


\section{Bibliography}

[AB15] Marcos M Alexandrino e Renato G. Bettiol. Lie Groups and Geometric Aspects of Isometric Actions. Springer International Publishing, 2015. 13

[ABS64] Michael F Atiyah, Raoul Bott e Arnold Shapiro. Clifford modules. Topology, 3:3-38, 1964. 16

[AR15] Marcos M Alexandrino e Marco Radeschi. Isometries between leaf spaces. Geometriae Dedicata, 174(1):193-201, 2015. 49

[AR16] Marcos M Alexandrino e Marco Radeschi. Closure of singular foliations: the proof of molino's conjecture. arXiv preprint arXiv:1608.03552, 2016. 2

[BBI01] Dmitri Burago, Yuri Burago e Sergei Ivanov. A course in metric geometry, volume 33. American Mathematical Society Providence, 2001. 46

[Car38] Élie Cartan. Familles de surfaces isoparamétriques dans les espaces à courbure constante. Annali di Matematica Pura ed Applicata, 17(1):177-191, 1938. 49

[Car39] Elie Cartan. Sur des familles remarquables d'hypersurfaces isoparamétriques dans les espaces sphériques. Mathematische Zeitschrift, 45(1):335-367, 1939. 49

[Cec13] T.E. Cecil. Lie Sphere Geometry: With Applications to Submanifolds. Universitext. Springer New York, 2013. 19, 20, 32, 33

[CR15] T.E. Cecil e P.J. Ryan. Geometry of Hypersurfaces. Springer Monographs in Mathematics. Springer New York, 2015. 2, 21, 23, 24, 32, 33, 49

[Dad85] Jiri Dadok. Polar coordinates induced by actions of compact lie groups. Transactions of the American Mathematical Society, 288(1):125-137, 1985. 59

[Dav10] Michael W. Davis. Lectures on orbifolds and reflection groups. https://www.researchgate.net/publication/241090965_Lectures_on_ orbifolds_and_reflection_groups, 2010. 67

[FKM81] Dirk Ferus, Hermann Karcher e Hans-Friedrich Münzner. Cliffordalgebren und neue isoparametrische hyperflächen. Mathematische Zeitschrift, 177(4):479-502, 1981. $1,3,15,20,32,33,44,50,56$

[Gal12] Jean Gallier. Clifford algebras, clifford groups, and a generalization of the quaternions: The pin and spin groups. Lecture Notes, University of Pennsylvania, Department of Computer and Information Science Preprint Series, 2012. 55

$\left[\mathrm{GG}^{+} 88\right]$ Detlef Gromoll, Karsten Grove et al. The low-dimensional metric foliations of euclidean spheres. Journal of Differential Geometry, 28(1):143-156, 1988. 44, 56,59

[GHL12] S. Gallot, D. Hulin e J. Lafontaine. Riemannian Geometry. Universitext. Springer Berlin Heidelberg, 2012. 8, 9, 65 
[GL16] Claudio Gorodski e Alexander Lytchak. Isometric actions on spheres with an orbifold quotient. Mathematische Annalen, 365(3-4):1041-1067, 2016. 58, 59

[Gor14] Claudio Gorodski. A metric approach to representations of compact lie groups. Lecture Notes, Ohio State University, Mathematics Research Institute Preprint Series, 2014. 67

[GR16] Claudio Gorodski e Marco Radeschi. On homogeneous composed clifford foliations. Münster Journal of Mathematics, 9:35-50, 2016. 1, 61

[GW07] Luis Guijarro e Gerard Walschap. When is a riemannian submersion homogeneous? Geometriae Dedicata, 125(1):47-52, 2007. 56

[GWZ $\left.{ }^{+} 08\right]$ Karsten Grove, Burkhard Wilking, Wolfgang Ziller et al. Positively curved cohomogeneity one manifolds and 3-sasakian geometry. Journal of Differential Geometry, 78(1):33-111, 2008. 56, 59

[Joh] Niles Johnson. A visualization of the hopf fibration. http://nilesjohnson.net/ hopf.html. 43

[LM89] H.B. Lawson e M.L. Michelsohn. Spin Geometry. Princeton Mathematical Series. Princeton University Press, 1989. 54

[LR15] Alexander Lytchak e Marco Radeschi. Algebraic nature of singular riemannian foliations in spheres. Journal für die reine und angewandte Mathematik (Crelles Journal), 2015. 1, 51

[LT10] Alexander Lytchak e Gudlaugur Thorbergsson. Curvature explosion in quotients and applications. Journal of Differential Geometry, 85(1):117-140, 2010. 14, 58

[LW16] Alexander Lytchak e Burkhard Wilking. Riemannian foliations of spheres. Geometry and Topology, 20(3):1257-1274, 2016. 56

[Lyt14] Alexander Lytchak. Polar foliations of symmetric spaces. Geometric and Functional Analysis, 24(4):1298-1315, 2014. 45

[MC88] Pierre Molino e Grant Cairns. Riemannian foliations. Birkhauser Boston Inc., 1988. 10, 13, 14

[MR16] Ricardo Mendes e Marco Radeschi. Singular riemannian foliations and their quadratic basic polynomials. arXiv preprint arXiv:1611.02067, 2016. 2, 51

[Mün80] Hans Friedrich Münzner. Isoparametrische hyperflächen in sphären I. Mathematische Annalen, 251(1):57-71, 1980. 49

[Mün81] Hans Friedrich Münzner. Isoparametrische hyperflächen in sphären II. Mathematische Annalen, 256(2):215-232, 1981. 49

[Rad12] Marco Radeschi. Low dimensional singular riemannian foliations in spheres. arXiv preprint arXiv:1203.6113, 2012. 60

[Rad14] Marco Radeschi. Clifford algebras and new singular riemannian foliations in spheres. Geometric and Functional Analysis, 24(5):1660-1682, 2014. 1, 2, 3, 5, $35,49,51,58,61$

[Str94] Eldar Straume. On the invariant theory and geometry of compact linear groups of cohomogeneity $\leqslant 3$. Differential Geometry and its Applications, 4(1):1-23, 1994. 59 
[Thu02] William P. Thurston. The geometry and topology of three-manifolds. http: //www.msri.org/publications/books/gt3m/, 2002. 67, 68

[Wil01] Burkhard Wilking. Index parity of closed geodesics and rigidity of Hopf fibrations. Inventiones mathematicae, 144(2):281-295, 2001. 44, 56 